Network Working Group

Request for Comments: 4340

E. Kohler

Category: Standards Track

M. Handley

UCLA
UCI

S. Floyd

ICIR

March 2006

\title{
Datagram Congestion Control Protocol (DCCP)
}

Status of This Memo

This document specifies an Internet standards track protocol for the Internet community, and requests discussion and suggestions for improvements. Please refer to the current edition of the "Internet Official Protocol Standards" (STD 1) for the standardization state and status of this protocol. Distribution of this memo is unlimited.

Copyright Notice

Copyright (C) The Internet Society (2006).

\section{Abstract}

The Datagram Congestion Control Protocol (DCCP) is a transport protocol that provides bidirectional unicast connections of congestion-controlled unreliable datagrams. DCCP is suitable for applications that transfer fairly large amounts of data and that can benefit from control over the tradeoff between timeliness and reliability.

Table of Contents

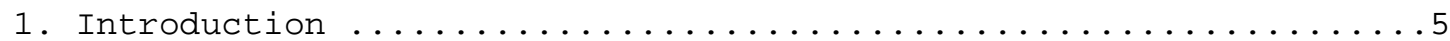

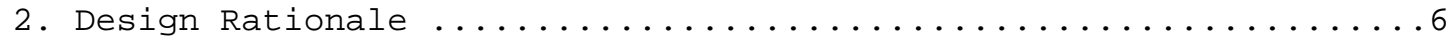

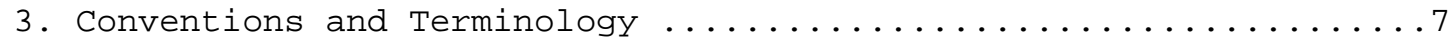

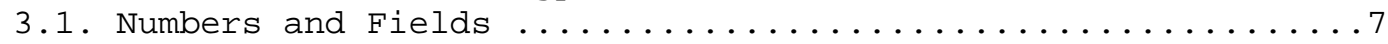

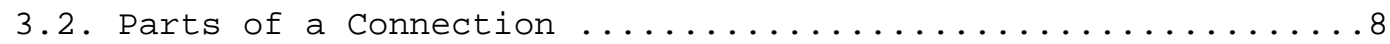

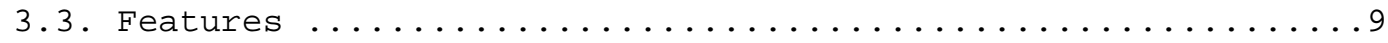

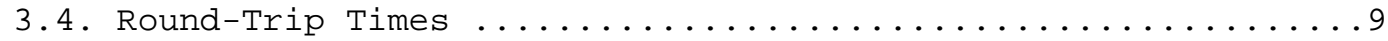

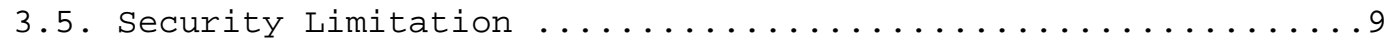

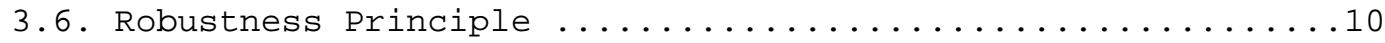

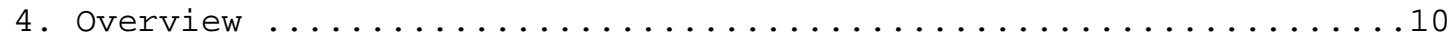

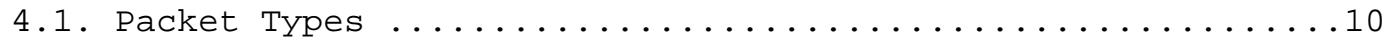

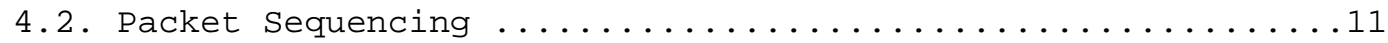

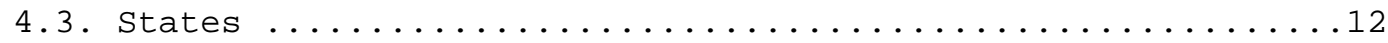

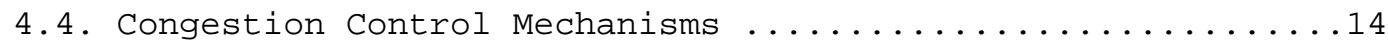




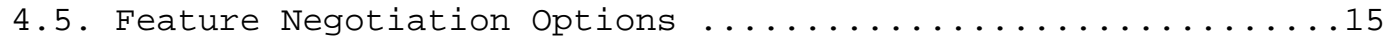

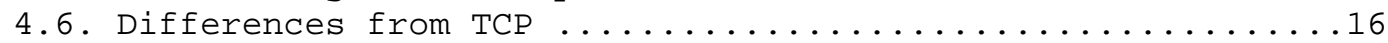

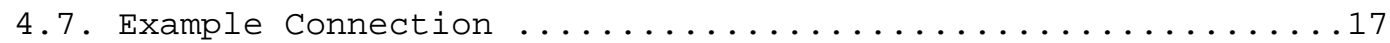

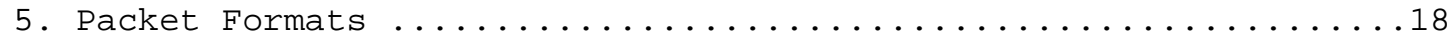

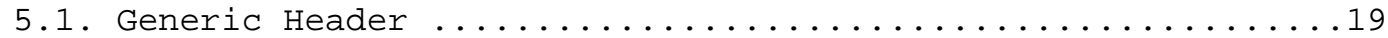

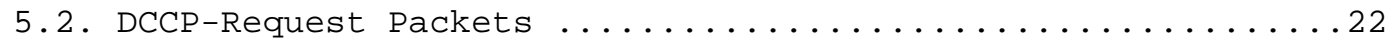

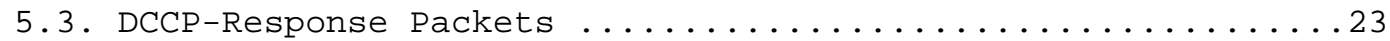

5.4. DCCP-Data, DCCP-Ack, and DCCP-DataAck Packets ........23

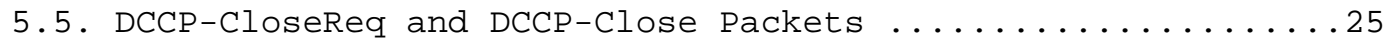

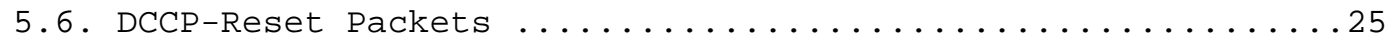

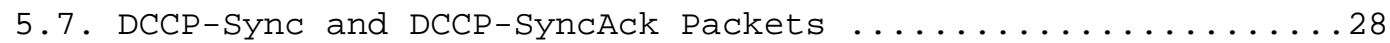

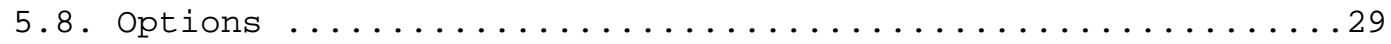

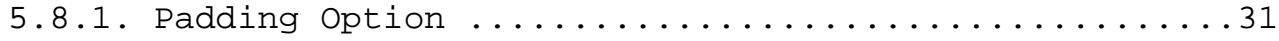

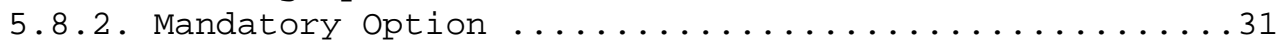

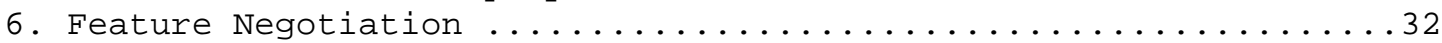

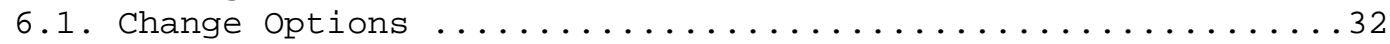

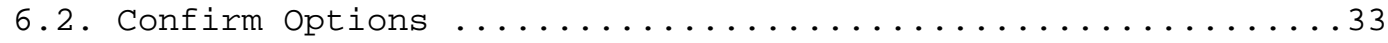

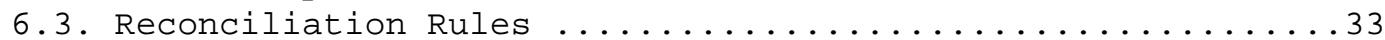

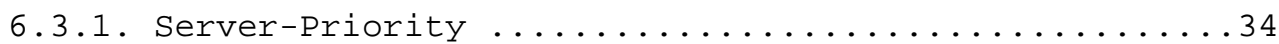

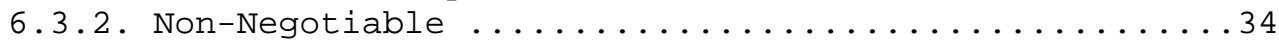

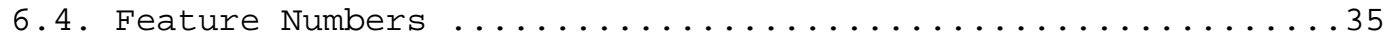

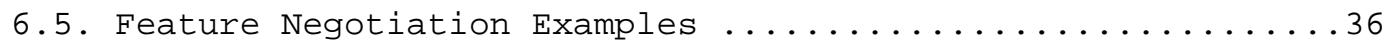

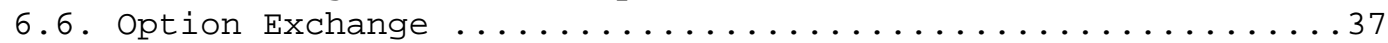

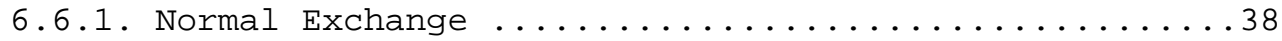

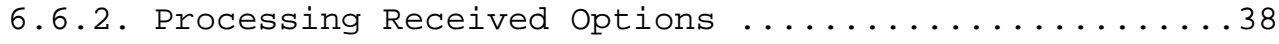

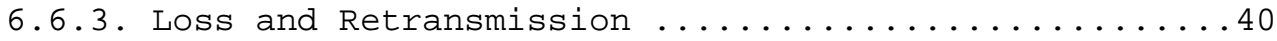

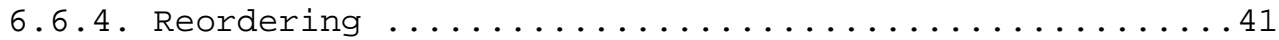

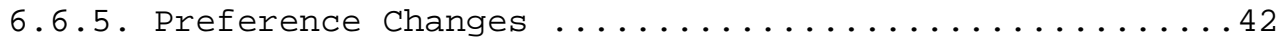

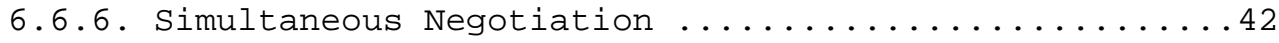

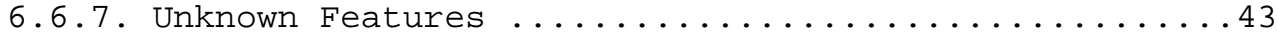

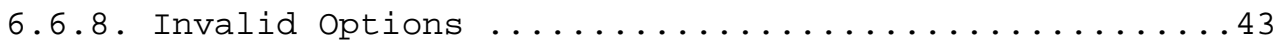

6.6.9. Mandatory Feature Negotiation ...............44

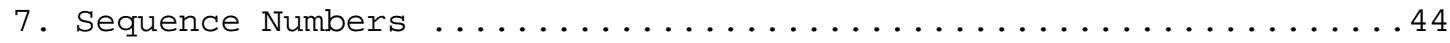

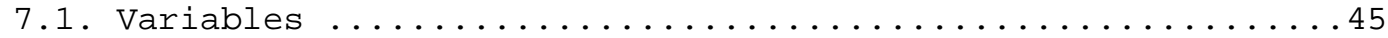

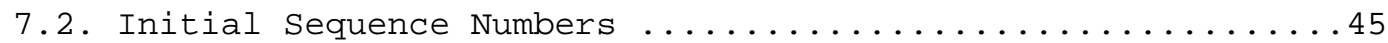

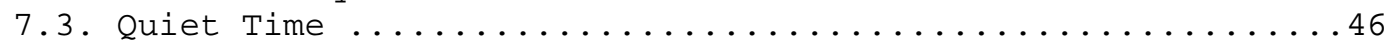

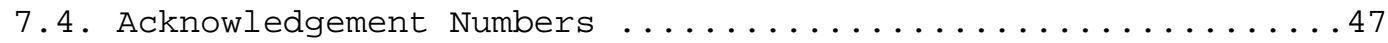

7.5. Validity and Synchronization ........................ 47

7.5.1. Sequence and Acknowledgement Number Windows .....48

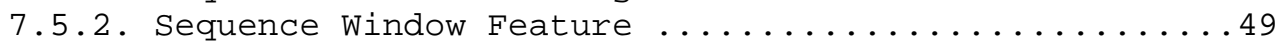

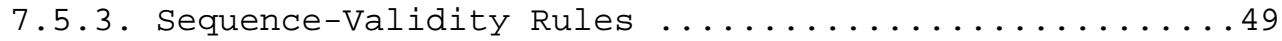

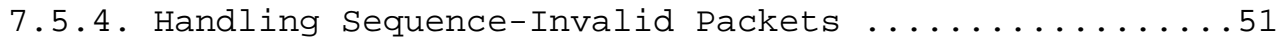

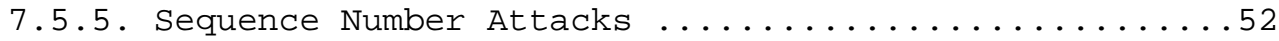

7.5.6. Sequence Number Handling Examples .............54

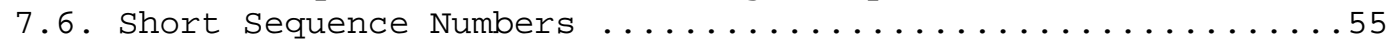

7.6.1. Allow Short Sequence Numbers Feature ............55

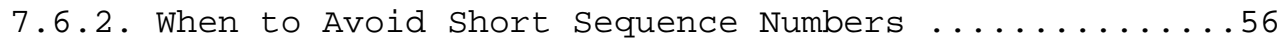

7.7. NDP Count and Detecting Application Loss ............56 


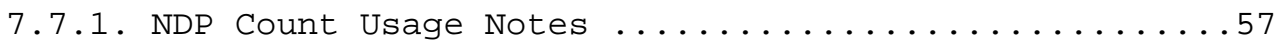

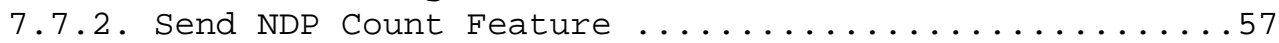

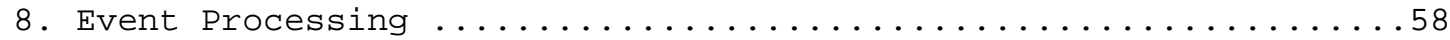

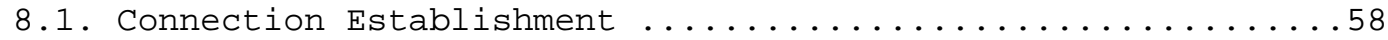

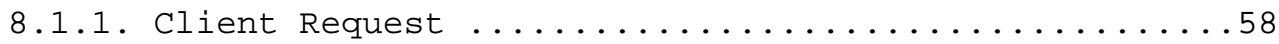

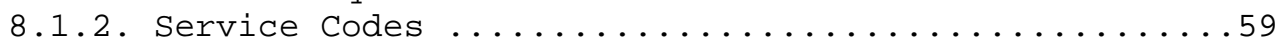

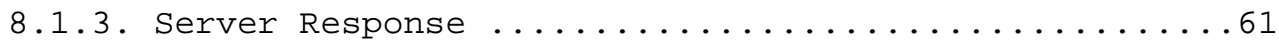

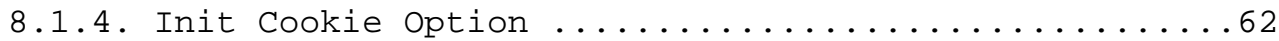

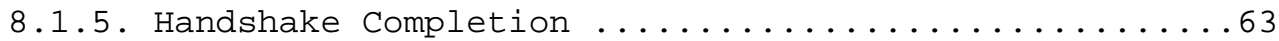

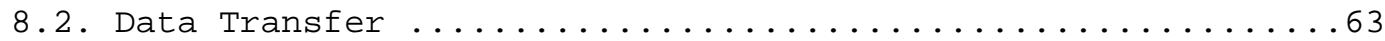

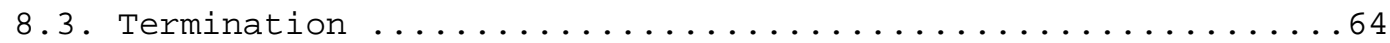

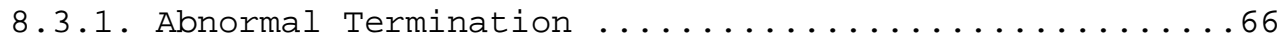

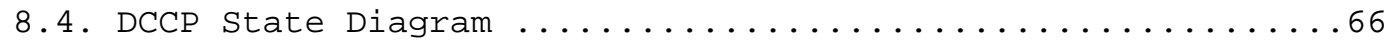

8.5. Pseudocode .........................67

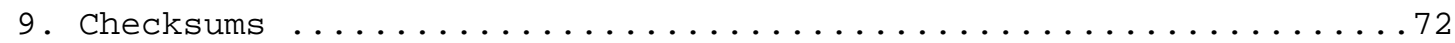

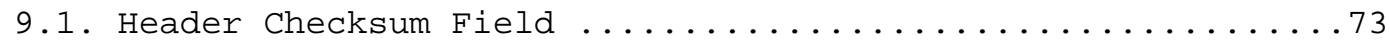

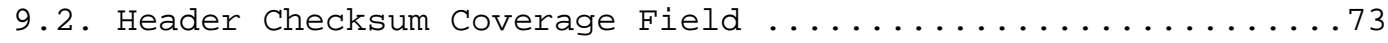

9.2.1. Minimum Checksum Coverage Feature ..........................

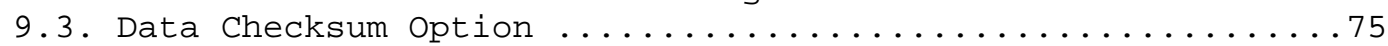

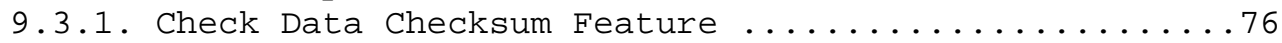

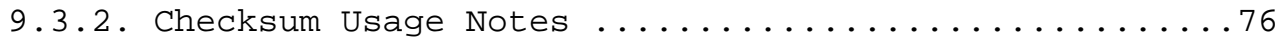

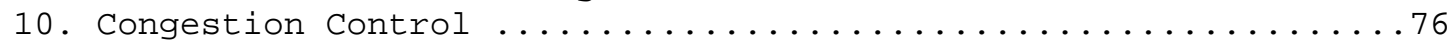

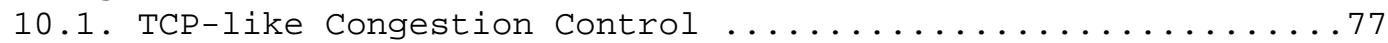

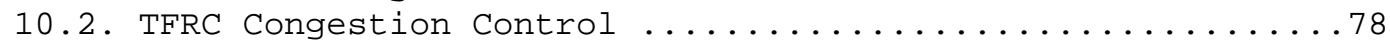

10.3. CCID-Specific Options, Features, and Reset Codes .......78

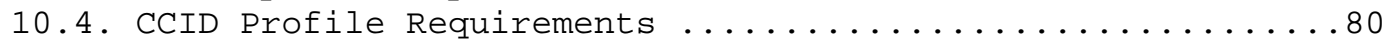

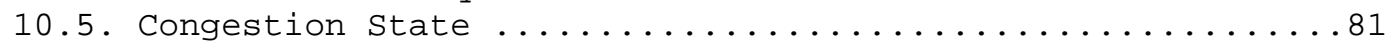

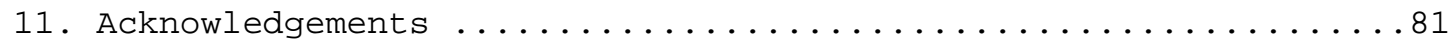

11.1. Acks of Acks and Unidirectional Connections .........82

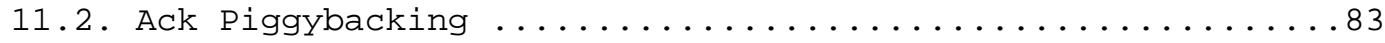

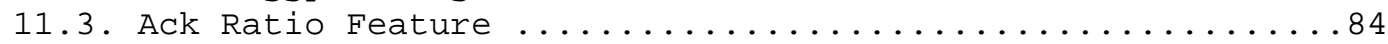

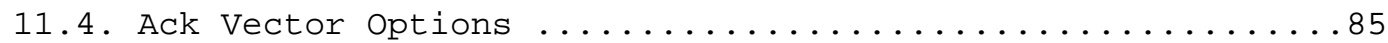

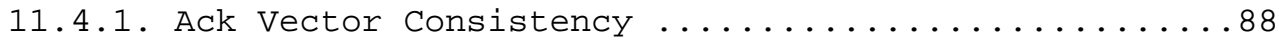

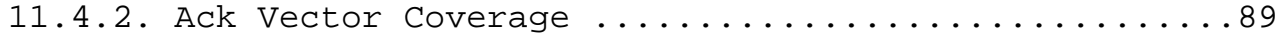

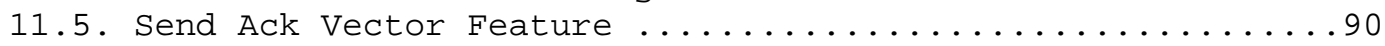

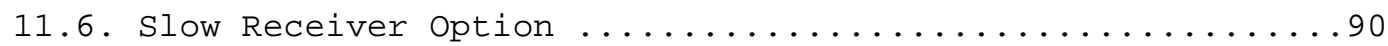

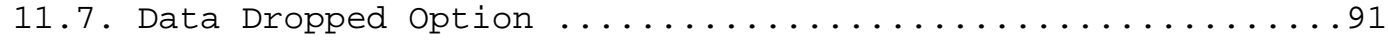

11.7.1. Data Dropped and Normal Congestion Response .....94

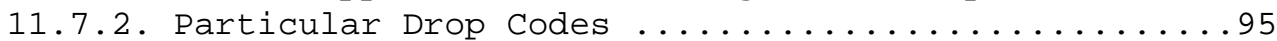

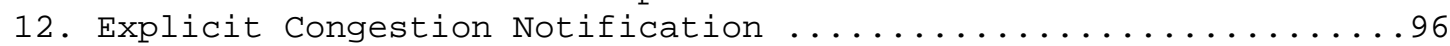

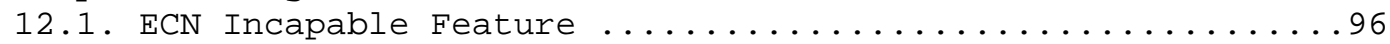

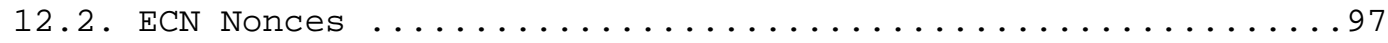

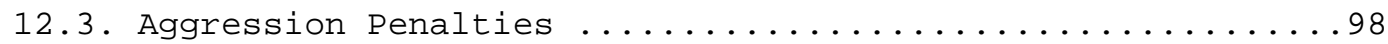

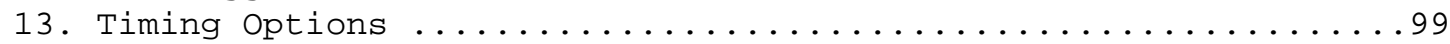

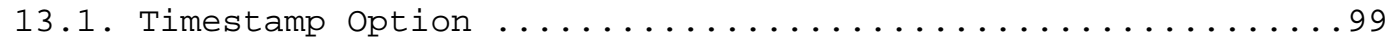

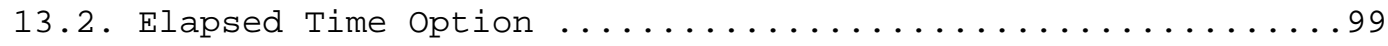

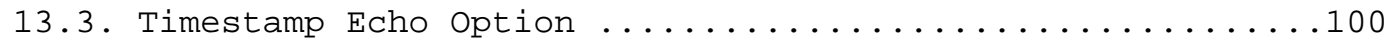

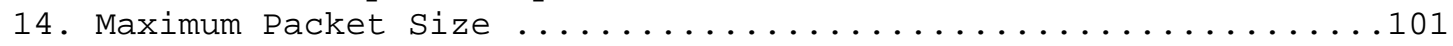




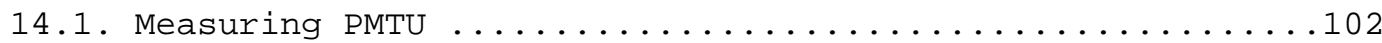

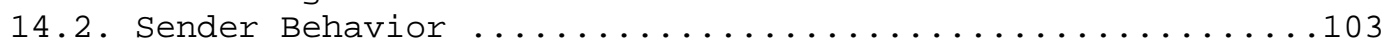

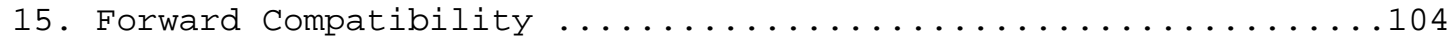

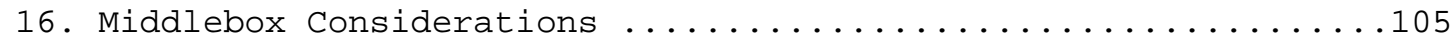

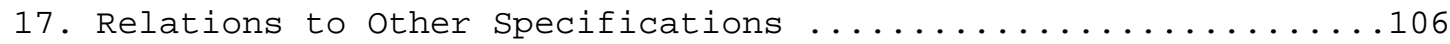

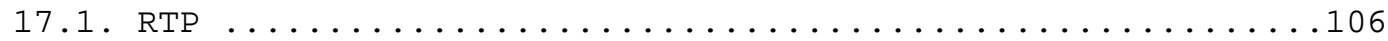

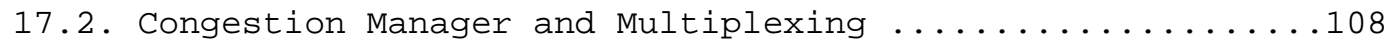

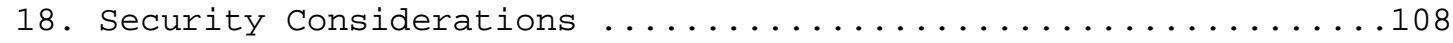

18.1. Security Considerations for Partial Checksums ........109

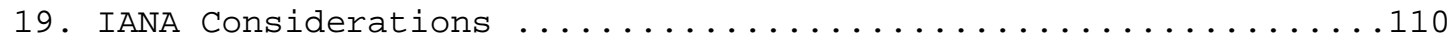

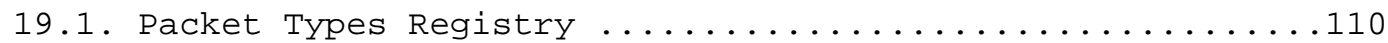

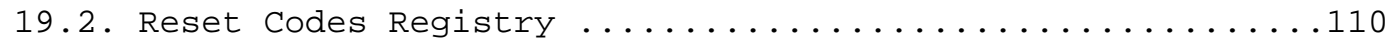

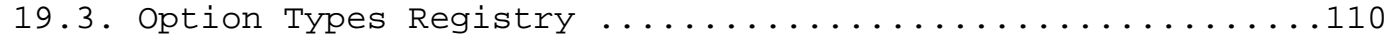

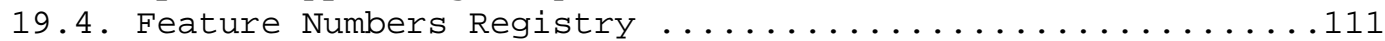

19.5. Congestion Control Identifiers Registry .............111

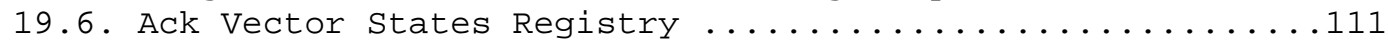

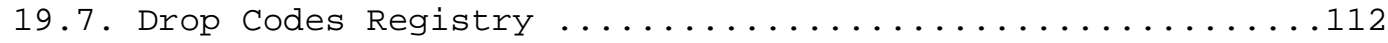

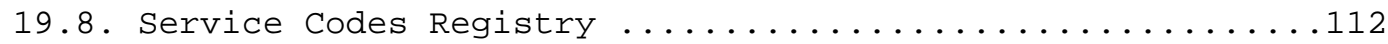

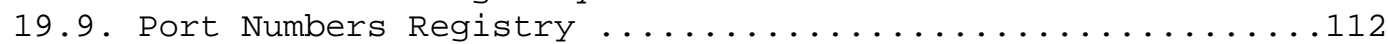

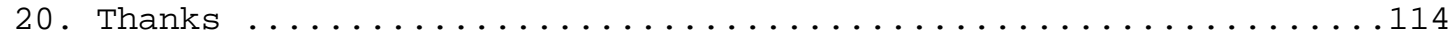

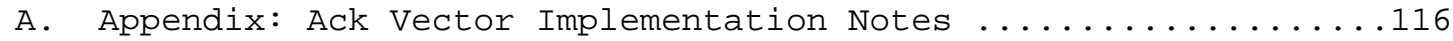

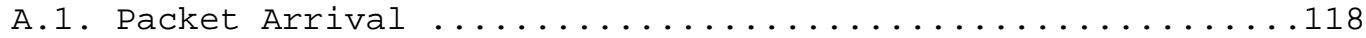

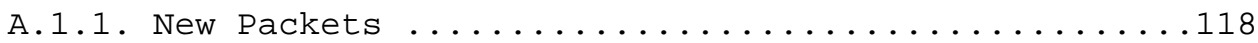

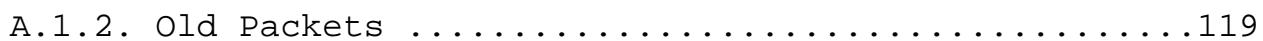

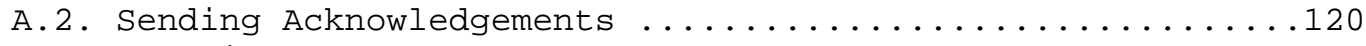

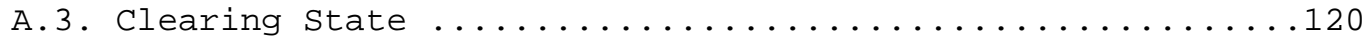

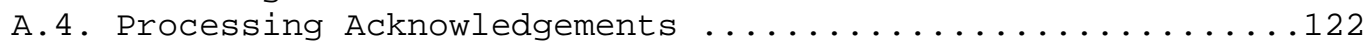

B. Appendix: Partial Checksumming Design Motivation .........123

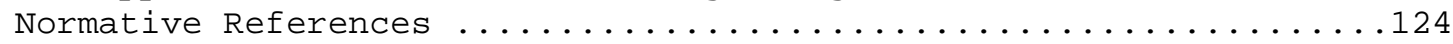

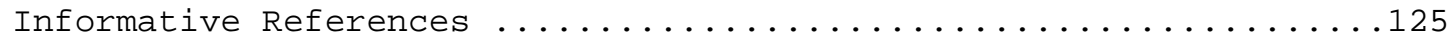

List of Tables

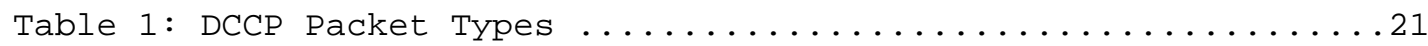

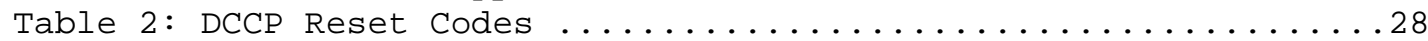

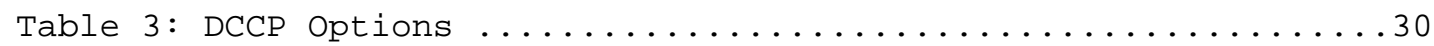

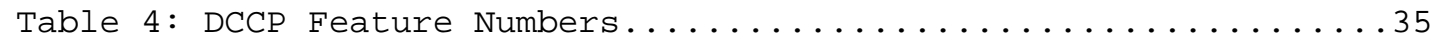

Table 5: DCCP Congestion Control Identifiers ................77

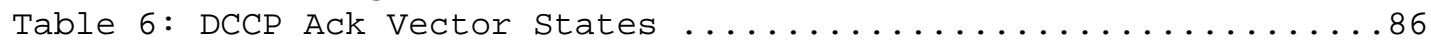

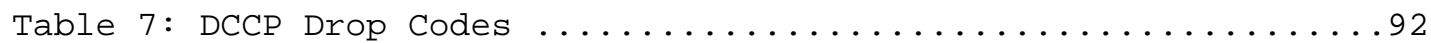




\section{Introduction}

The Datagram Congestion Control Protocol (DCCP) is a transport protocol that implements bidirectional, unicast connections of congestion-controlled, unreliable datagrams. Specifically, DCCP provides the following:

- Unreliable flows of datagrams.

- Reliable handshakes for connection setup and teardown.

- Reliable negotiation of options, including negotiation of a suitable congestion control mechanism.

- Mechanisms allowing servers to avoid holding state for unacknowledged connection attempts and already-finished connections.

- Congestion control incorporating Explicit Congestion Notification (ECN) [RFC3168] and the ECN Nonce [RFC3540].

- Acknowledgement mechanisms communicating packet loss and ECN information. Acks are transmitted as reliably as the relevant congestion control mechanism requires, possibly completely reliably.

- Optional mechanisms that tell the sending application, with high reliability, which data packets reached the receiver, and whether those packets were ECN marked, corrupted, or dropped in the receive buffer.

o Path Maximum Transmission Unit (PMTU) discovery [RFC1191].

- A choice of modular congestion control mechanisms. Two mechanisms are currently specified: TCP-like Congestion Control [RFC4341] and TCP-Friendly Rate Control (TFRC) [RFC4342]. DCCP is easily extensible to further forms of unicast congestion control.

DCCP is intended for applications such as streaming media that can benefit from control over the tradeoffs between delay and reliable in-order delivery. TCP is not well suited for these applications, since reliable in-order delivery and congestion control can cause arbitrarily long delays. UDP avoids long delays, but UDP applications that implement congestion control must do so on their own. DCCP provides built-in congestion control, including ECN 
support, for unreliable datagram flows, avoiding the arbitrary delays associated with TCP. It also implements reliable connection setup, teardown, and feature negotiation.

\section{Design Rationale}

One DCCP design goal was to give most streaming UDP applications little reason not to switch to DCCP, once it is deployed. To facilitate this, DCCP was designed to have as little overhead as possible, both in terms of the packet header size and in terms of the state and $\mathrm{CPU}$ overhead required at end hosts. Only the minimal necessary functionality was included in DCCP, leaving other functionality, such as forward error correction (FEC), semireliability, and multiple streams, to be layered on top of DCCP as desired.

Different forms of conformant congestion control are appropriate for different applications. For example, on-line games might want to make quick use of any available bandwidth, while streaming media might trade off this responsiveness for a steadier, less bursty rate. (Sudden rate changes can cause unacceptable UI glitches such as audible pauses or clicks in the playout stream.) DCCP thus allows applications to choose from a set of congestion control mechanisms. One alternative, TCP-like Congestion Control, halves the congestion window in response to a packet drop or mark, as in TCP. Applications using this congestion control mechanism will respond quickly to changes in available bandwidth, but must tolerate the abrupt changes in congestion window typical of TCP. A second alternative, TCPFriendly Rate Control (TFRC) [RFC3448], a form of equation-based congestion control, minimizes abrupt changes in the sending rate while maintaining longer-term fairness with TCP. Other alternatives can be added as future congestion control mechanisms are standardized.

DCCP also lets unreliable traffic safely use ECN. A UDP kernel Application Programming Interface (API) might not allow applications to set UDP packets as ECN capable, since the API could not guarantee that the application would properly detect or respond to congestion. DCCP kernel APIs will have no such issues, since DCCP implements congestion control itself.

We chose not to require the use of the Congestion Manager [RFC3124], which allows multiple concurrent streams between the same sender and receiver to share congestion control. The current Congestion Manager can only be used by applications that have their own end-to-end feedback about packet losses, but this is not the case for many of the applications currently using UDP. In addition, the current Congestion Manager does not easily support multiple congestion 
control mechanisms or mechanisms where the state about past packet drops or marks is maintained at the receiver rather than the sender. DCCP should be able to make use of CM where desired by the application, but we do not see any benefit in making the deployment of DCCP contingent on the deployment of CM itself.

We intend for DCCP's protocol mechanisms, which are described in this document, to suit any application desiring unicast congestion-

controlled streams of unreliable datagrams. However, the congestion control mechanisms currently approved for use with DCCP, which are described in separate Congestion Control ID Profiles [RFC4341, RFC4342], may cause problems for some applications, including highbandwidth interactive video. These applications should be able to use DCCP once suitable Congestion Control ID Profiles are standardized.

3. Conventions and Terminology

The key words "MUST", "MUST NOT", "REQUIRED", "SHALL", "SHALL NOT", "SHOULD", "SHOULD NOT", "RECOMMENDED", "MAY", and "OPTIONAL" in this document are to be interpreted as described in [RFC2119].

\subsection{Numbers and Fields}

All multi-byte numerical quantities in DCCP, such as port numbers, Sequence Numbers, and arguments to options, are transmitted in network byte order (most significant byte first).

We occasionally refer to the "left" and "right" sides of a bit field. "Left" means towards the most significant bit, and "right" means towards the least significant bit.

Random numbers in DCCP are used for their security properties and SHOULD be chosen according to the guidelines in [RFC4086].

All operations on DCCP sequence numbers use circular arithmetic modulo 2^48, as do comparisons such as "greater" and "greatest". This form of arithmetic preserves the relationships between sequence numbers as they roll over from $2^{\wedge} 48-1$ to 0 . Implementation strategies for DCCP sequence numbers will resemble those for other circular arithmetic spaces, including $\mathrm{TCP}^{\prime} \mathrm{s}$ sequence numbers [RFC793] and DNS's serial numbers [RFC1982]. It may make sense to store DCCP sequence numbers in the most significant 48 bits of 64-bit integers and set the least significant 16 bits to zero, since this supports a common technique that implements circular comparison $A<B$ by testing whether $(A-B)<0$ using conventional two's-complement arithmetic. 
Reserved bitfields in DCCP packet headers MUST be set to zero by senders and MUST be ignored by receivers, unless otherwise specified. This allows for future protocol extensions. In particular, DCCP processors MUST NOT reset a DCCP connection simply because a Reserved field has non-zero value [RFC3360].

\subsection{Parts of a Connection}

Each DCCP connection runs between two hosts, which we often name DCCP $A$ and DCCP B. Each connection is actively initiated by one of the hosts, which we call the client; the other, initially passive host is called the server. The term "DCCP endpoint" is used to refer to either of the two hosts explicitly named by the connection (the client and the server). The term "DCCP processor" refers more generally to any host that might need to process a DCCP header; this includes the endpoints and any middleboxes on the path, such as firewalls and network address translators.

DCCP connections are bidirectional: data may pass from either endpoint to the other. This means that data and acknowledgements may flow in both directions simultaneously. Logically, however, a DCCP connection consists of two separate unidirectional connections, called half-connections. Each half-connection consists of the application data sent by one endpoint and the corresponding acknowledgements sent by the other endpoint. We can illustrate this as follows:

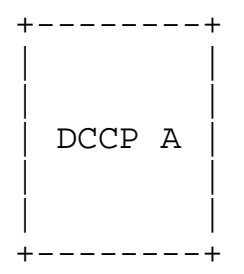

$$
\begin{gathered}
\text { A-to-B half-connection: } \\
-->\text { application data } \\
<-- \text { acknowledgements } \\
\text { B-to-A half-connection: } \\
\text { <-- application data } \\
\text {--> acknowledgements }
\end{gathered}
$$

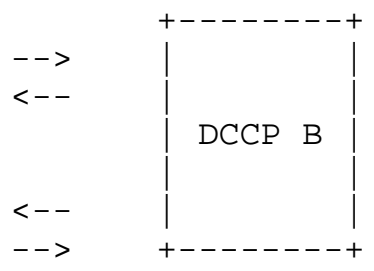

Although they are logically distinct, in practice the halfconnections overlap; a DCCP-DataAck packet, for example, contains application data relevant to one half-connection and acknowledgement information relevant to the other.

In the context of a single half-connection, the terms "HC-Sender" and "HC-Receiver" denote the endpoints sending application data and acknowledgements, respectively. For example, DCCP A is the HC-Sender and DCCP B is the HC-Receiver in the A-to-B half-connection. 


\subsection{Features}

A DCCP feature is a connection attribute on whose value the two endpoints agree. Many properties of a DCCP connection are controlled by features, including the congestion control mechanisms in use on the two half-connections. The endpoints achieve agreement through the exchange of feature negotiation options in DCCP headers.

DCCP features are identified by a feature number and an endpoint. The notation "F/X" represents the feature with feature number $F$ located at DCCP endpoint $X$. Each valid feature number thus corresponds to two features, which are negotiated separately and need not have the same value. The two endpoints know, and agree on, the value of every valid feature. DCCP A is the "feature location" for all features $F / A$, and the "feature remote" for all features $F / B$.

\subsection{Round-Trip Times}

DCCP round-trip time measurements are performed by congestion control mechanisms; different mechanisms may measure round-trip time in different ways, or not measure it at all. However, the main DCCP protocol does use round-trip times occasionally, such as in the initial values for certain timers. Each DCCP implementation thus defines a default round-trip time for use when no estimate is available. This parameter should default to not less than 0.2 seconds, a reasonably conservative round-trip time for Internet TCP connections. Protocol behavior specified in terms of "round-trip time" values actually refers to "a current round-trip time estimate taken by some CCID, or, if no estimate is available, the default round-trip time parameter".

The maximum segment lifetime, or MSL, is the maximum length of time a packet can survive in the network. The DCCP MSL should equal that of TCP, which is normally two minutes.

\subsection{Security Limitation}

DCCP provides no protection against attackers who can snoop on a connection in progress, or who can guess valid sequence numbers in other ways. Applications desiring stronger security should use IPsec [RFC2401]; depending on the level of security required, applicationlevel cryptography may also suffice. These issues are discussed further in Sections 7.5 .5 and 18 . 


\subsection{Robustness Principle}

DCCP implementations will follow TCP's "general principle of robustness": "be conservative in what you do, be liberal in what you accept from others" [RFC793].

4. Overview

DCCP's high-level connection dynamics echo those of TCP. Connections progress through three phases: initiation, including a three-way handshake; data transfer; and termination. Data can flow both ways over the connection. An acknowledgement framework lets senders discover how much data has been lost and thus avoid unfairly congesting the network. Of course, DCCP provides unreliable datagram semantics, not TCP's reliable bytestream semantics. The application must package its data into explicit frames and must retransmit its own data as necessary. It may be useful to think of DCCP as TCP minus bytestream semantics and reliability, or as UDP plus congestion control, handshakes, and acknowledgements.

\subsection{Packet Types}

Ten packet types implement DCCP's protocol functions. For example, every new connection attempt begins with a DCCP-Request packet sent by the client. In this way a DCCP-Request packet resembles a TCP SYN, but since DCCP-Request is a packet type there is no way to send an unexpected flag combination, such as TCP's SYN+FIN+ACK+RST.

Eight packet types occur during the progress of a typical connection, shown here. Note the three-way handshakes during initiation and termination.

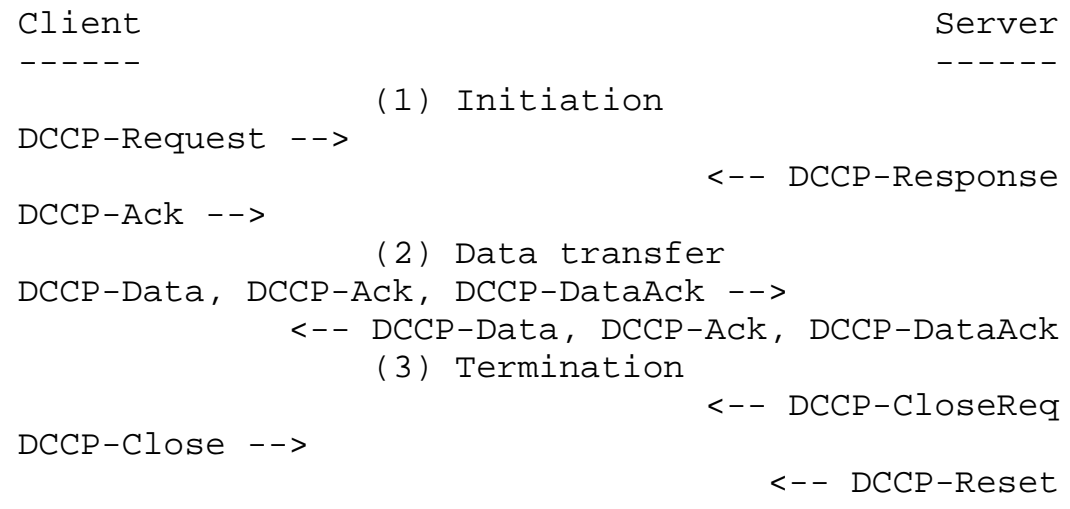

The two remaining packet types are used to resynchronize after bursts of loss. 
Every DCCP packet starts with a fixed-size generic header.

Particular packet types include additional fixed-size header data; for example, DCCP-Acks include an Acknowledgement Number. DCCP options and any application data follow the fixed-size header.

The packet types are as follows:

DCCP-Request

Sent by the client to initiate a connection (the first part of the three-way initiation handshake).

DCCP-Response Sent by the server in response to a DCCP-Request (the second part of the three-way initiation handshake).

DCCP-Data

Used to transmit application data.

$\mathrm{DCCP}-\mathrm{ACk}$

Used to transmit pure acknowledgements.

DCCP-DataAck

Used to transmit application data with piggybacked acknowledgement information.

DCCP-CloseReg

Sent by the server to request that the client close the connection.

DCCP-Close

Used by the client or the server to close the connection; elicits a DCCP-Reset in response.

DCCP-Reset

Used to terminate the connection, either normally or abnormally.

DCCP-Sync, DCCP-SyncAck

Used to resynchronize sequence numbers after large bursts of loss.

4.2. Packet Sequencing

Each DCCP packet carries a sequence number so that losses can be detected and reported. Unlike TCP sequence numbers, which are bytebased, DCCP sequence numbers increment by one per packet. For example: 


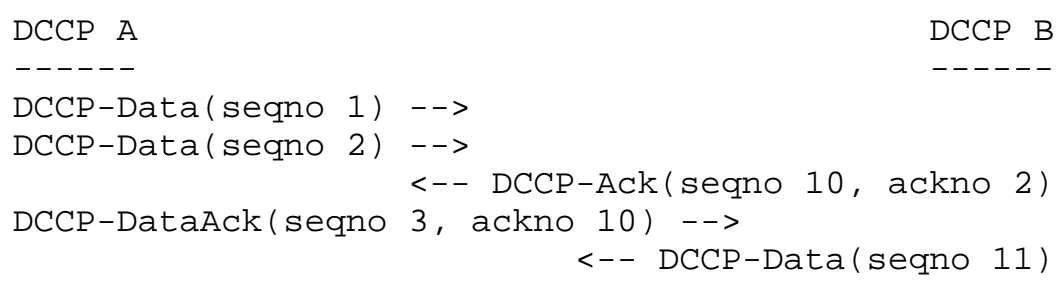

Every DCCP packet increments the sequence number, whether or not it contains application data. DCCP-Ack pure acknowledgements increment the sequence number; for instance, DCCP $B^{\prime}$ s second packet above uses sequence number 11, since sequence number 10 was used for an acknowledgement. This lets endpoints detect all packet loss, including acknowledgement loss. It also means that endpoints can get out of sync after long bursts of loss. The DCCP-Sync and DCCPSyncAck packet types are used to recover (Section 7.5).

Since DCCP provides unreliable semantics, there are no retransmissions, and having a TCP-style cumulative acknowledgement field doesn't make sense. DCCP's Acknowledgement Number field equals the greatest sequence number received, rather than the smallest sequence number not received. Separate options indicate any intermediate sequence numbers that weren't received.

4.3. States

DCCP endpoints progress through different states during the course of a connection, corresponding roughly to the three phases of initiation, data transfer, and termination. The figure below shows the typical progress through these states for a client and server. 
Client $------$

CLOSED

REQUEST

PARTOPEN

OPEN

CLOSING

TIMEWAIT

CLOSED
Server

$------$

LISTEN

RESPOND

(1) Initiation DCCP-Request $-->$ $<--$ DCCP-Response DCCP-Ack or DCCP-DataAck -->

(2) Data transfer <-- DCCP-Data, Ack, DataAck --> OPEN

(3) Termination $<--$ DCCP-CloseReq CLOSEREQ DCCP-Close $-->$ $<--$ DCCP-Reset CLOSED

The nine possible states are as follows. They are listed in increasing order, so that "state $>=$ CLOSEREQ" means the same as "state $=$ CLOSEREQ or state $=$ CLOSING or state = TIMEWAIT". Section 8 describes the states in more detail.

\section{CLOSED}

Represents nonexistent connections.

LISTEN

Represents server sockets in the passive listening state. LISTEN and CLOSED are not associated with any particular DCCP connection. REQUEST

A client socket enters this state, from CLOSED, after sending a DCCP-Request packet to try to initiate a connection.

RESPOND

A server socket enters this state, from LISTEN, after receiving a DCCP-Request from a client.

PARTOPEN

A client socket enters this state, from REQUEST, after receiving a DCCP-Response from the server. This state represents the third phase of the three-way handshake. The client may send application data in this state, but it MUST include an Acknowledgement Number on all of its packets. 
OPEN

The central data transfer portion of a DCCP connection. Client and server sockets enter this state from PARTOPEN and RESPOND, respectively. Sometimes we speak of SERVER-OPEN and CLIENT-OPEN states, corresponding to the server's OPEN state and the client's OPEN state.

\section{CLOSEREQ}

A server socket enters this state, from SERVER-OPEN, to order the client to close the connection and to hold TIMEWAIT state.

\section{CLOSING}

Server and client sockets can both enter this state to close the connection.

\section{TIMEWAIT}

A server or client socket remains in this state for 2MSL (4 minutes) after the connection has been torn down, to prevent mistakes due to the delivery of old packets. Only one of the endpoints has to enter TIMEWAIT state (the other can enter CLOSED state immediately), and a server can request its client to hold TIMEWAIT state using the DCCP-CloseReq packet type.

\subsection{Congestion Control Mechanisms}

DCCP connections are congestion controlled, but unlike in TCP, DCCP applications have a choice of congestion control mechanism. In fact, the two half-connections can be governed by different mechanisms. Mechanisms are denoted by one-byte congestion control identifiers, or CCIDs. The endpoints negotiate their CCIDs during connection initiation. Each CCID describes how the HC-Sender limits data packet rates, how the HC-Receiver sends congestion feedback via acknowledgements, and so forth. CCIDs 2 and 3 are currently defined; CCIDs 0, 1, and 4-255 are reserved. Other CCIDs may be defined in the future.

CCID 2 provides TCP-like Congestion Control, which is similar to that of TCP. The sender maintains a congestion window and sends packets until that window is full. Packets are acknowledged by the receiver. Dropped packets and ECN [RFC3168] indicate congestion; the response to congestion is to halve the congestion window. Acknowledgements in CCID 2 contain the sequence numbers of all received packets within some window, similar to a selective acknowledgement (SACK) [RFC2018].

CCID 3 provides TCP-Friendly Rate Control (TFRC), an equation-based form of congestion control intended to respond to congestion more smoothly than CCID 2. The sender maintains a transmit rate, which it updates using the receiver's estimate of the packet loss and mark 
rate. CCID 3 behaves somewhat differently than TCP in the short

term, but is designed to operate fairly with TCP over the long term.

Section 10 describes DCCP's CCIDs in more detail. The behaviors of CCIDs 2 and 3 are fully defined in separate profile documents [RFC4341, RFC4342].

\subsection{Feature Negotiation Options}

DCCP endpoints use Change and Confirm options to negotiate and agree on feature values. Feature negotiation will almost always happen on the connection initiation handshake, but it can begin at any time.

There are four feature negotiation options in all: Change L, Confirm $L$, Change R, and Confirm R. The "L" options are sent by the feature location and the "R" options are sent by the feature remote. A Change $R$ option says to the feature location, "change this feature value as follows". The feature location responds with Confirm $\mathrm{L}$, meaning, "I've changed it". Some features allow Change R options to contain multiple values sorted in preference order. For example:

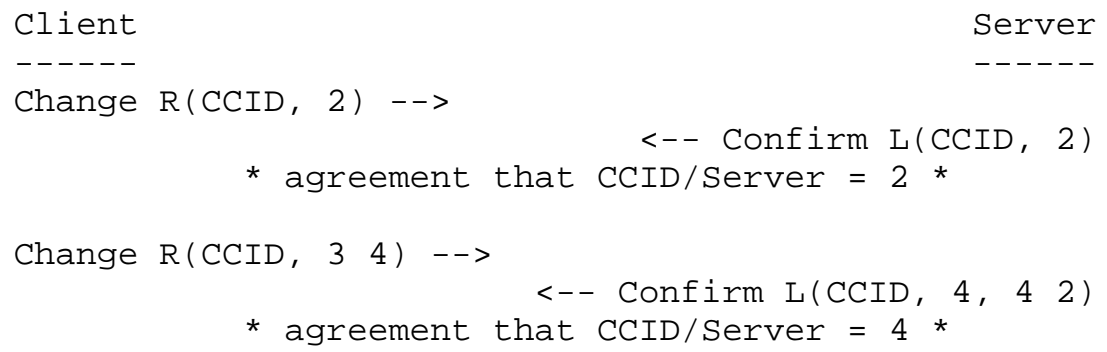




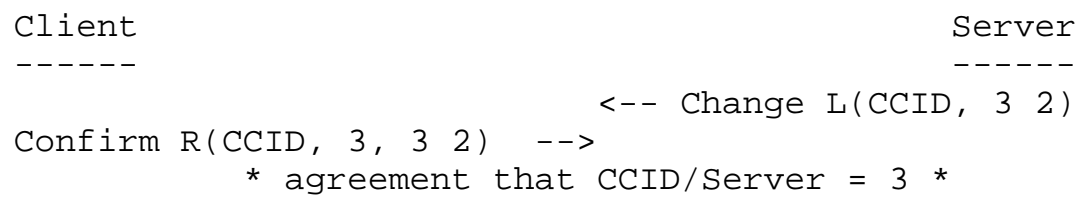

Section 6 describes the feature negotiation options further, including the retransmission strategies that make negotiation reliable.

4.6. Differences from TCP

DCCP's differences from TCP apart from those discussed so far include the following:

- Copious space for options (up to 1008 bytes or the PMTU) .

- Different acknowledgement formats. The CCID for a connection determines how much acknowledgement information needs to be transmitted. For example, in CCID 2 (TCP-like), this is about one ack per 2 packets, and each ack must declare exactly which packets were received. In CCID 3 (TFRC), it is about one ack per roundtrip time, and acks must declare at minimum just the lengths of recent loss intervals.

- Denial of Service (DoS) protection. Several mechanisms help limit the amount of state that possibly-misbehaving clients can force DCCP servers to maintain. An Init Cookie option analogous to TCP'S SYN Cookies [SYNCOOKIES] avoids SYN-flood-like attacks. Only one connection endpoint has to hold TIMEWAIT state; the DCCP-CloseReq packet, which may only be sent by the server, passes that state to the client. Various rate limits let servers avoid attacks that might force extensive computation or packet generation.

- Distinguishing different kinds of loss. A Data Dropped option (Section 11.7) lets an endpoint declare that a packet was dropped because of corruption, because of receive buffer overflow, and so on. This facilitates research into more appropriate rate-control responses for these non-network-congestion losses (although currently such losses will cause a congestion response).

- Acknowledgeability. In TCP, a packet may be acknowledged only once the data is reliably queued for application delivery. This does not make sense in DCCP, where an application might, for example, request a drop-from-front receive buffer. A DCCP packet may be acknowledged as soon as its header has been successfully processed. Concretely, a packet becomes acknowledgeable at step 8 
of Section 8.5's packet processing pseudocode. Acknowledgeability does not guarantee data delivery, however: the Data Dropped option may later report that the packet's application data was discarded.

- No receive window. DCCP is a congestion control protocol, not a flow control protocol.

- No simultaneous open. Every connection has one client and one server.

- No half-closed states. DCCP has no states corresponding to TCP's FINWAIT and CLOSEWAIT, where one half-connection is explicitly closed while the other is still active. The Data Dropped option's Drop Code 1, Application Not Listening (Section 11.7), can achieve a similar effect, however.

\subsection{Example Connection}

The progress of a typical DCCP connection is as follows. (This description is informative, not normative.)

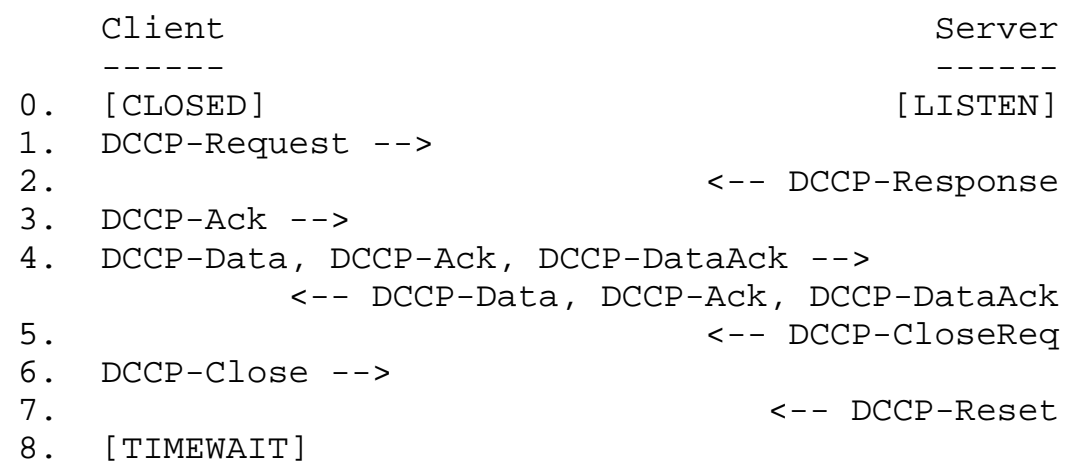

1. The client sends the server a DCCP-Request packet specifying the client and server ports, the service being requested, and any features being negotiated, including the CCID that the client would like the server to use. The client may optionally piggyback an application request on the DCCP-Request packet. The server may ignore this application request.

2. The server sends the client a DCCP-Response packet indicating that it is willing to communicate with the client. This response indicates any features and options that the server agrees to, begins other feature negotiations as desired, and optionally includes Init cookies that wrap up all this information and that must be returned by the client for the connection to complete. 
3. The client sends the server a DCCP-Ack packet that acknowledges the DCCP-Response packet. This acknowledges the server's initial sequence number and returns any Init Cookies in the DCCP-Response. It may also continue feature negotiation. The client may piggyback an application-level request on this ack, producing a DCCP-DataAck packet.

4. The server and client then exchange DCCP-Data packets, DCCP-ACk packets acknowledging that data, and, optionally, DCCP-DataAck packets containing data with piggybacked acknowledgements. If the client has no data to send, then the server will send DCCP-Data and DCCP-DataAck packets, while the client will send DCCP-Acks exclusively. (However, the client may not send DCCP-Data packets before receiving at least one non-DCCP-Response packet from the server.)

5. The server sends a DCCP-CloseReq packet requesting a close.

6. The client sends a DCCP-Close packet acknowledging the close.

7. The server sends a DCCP-Reset packet with Reset Code 1, "Closed", and clears its connection state. DCCP-Resets are part of normal connection termination; see Section 5.6.

8. The client receives the DCCP-Reset packet and holds state for two maximum segment lifetimes, or 2MSL, to allow any remaining packets to clear the network.

An alternative connection closedown sequence is initiated by the client:

5b. The client sends a DCCP-Close packet closing the connection.

6b. The server sends a DCCP-Reset packet with Reset Code 1, "Closed", and clears its connection state.

7b. The client receives the DCCP-Reset packet and holds state for 2MSL to allow any remaining packets to clear the network.

5. Packet Formats

The DCCP header can be from 12 to 1020 bytes long. The initial part of the header has the same semantics for all currently defined packet types. Following this comes any additional fixed-length fields required by the packet type, and then a variable-length list of options. The application data area follows the header. In some packet types, this area contains data for the application; in other packet types, its contents are ignored. 


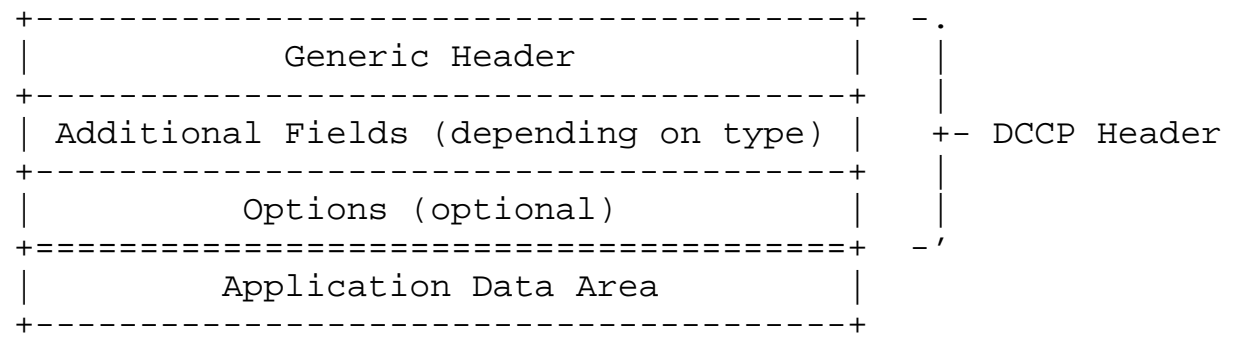

\subsection{Generic Header}

The DCCP generic header takes different forms depending on the value of $X$, the Extended Sequence Numbers bit. If $X$ is one, the Sequence Number field is 48 bits long, and the generic header takes 16 bytes, as follows.

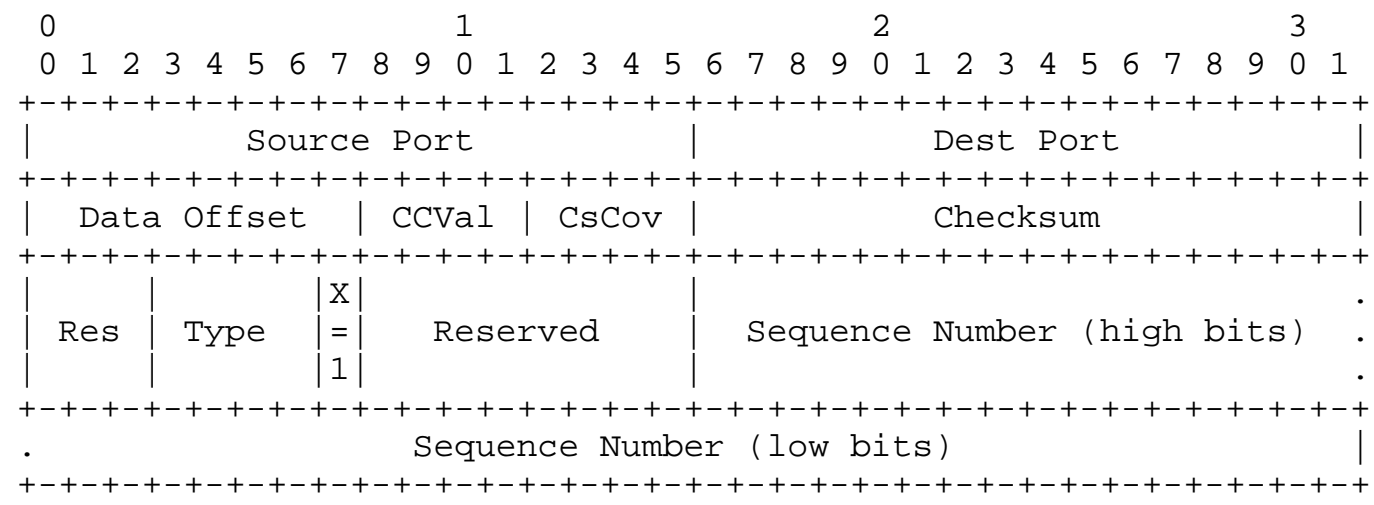

If $X$ is zero, only the low 24 bits of the sequence Number are transmitted, and the generic header is 12 bytes long.

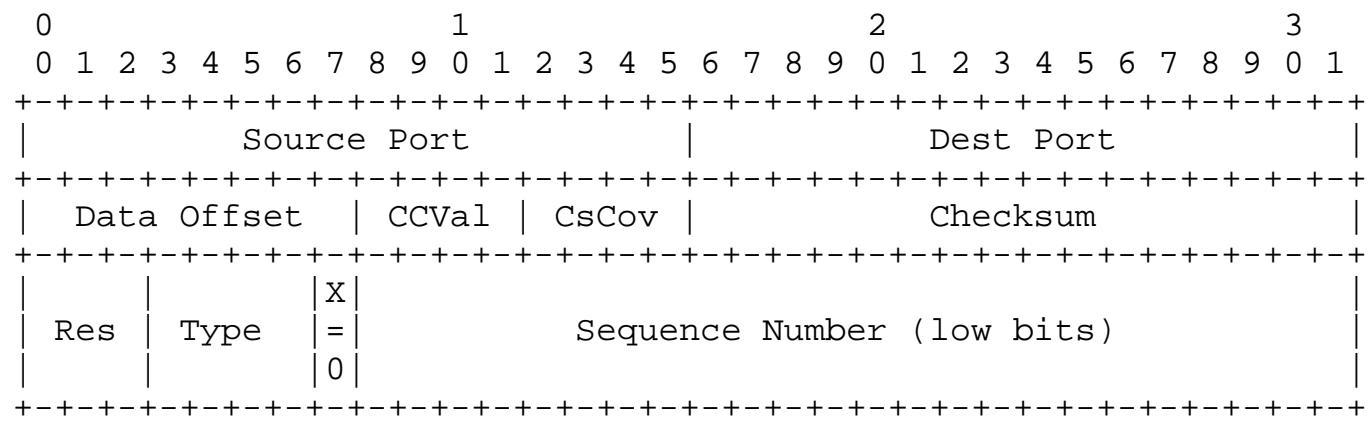


The generic header fields are defined as follows.

Source and Destination Ports: 16 bits each

These fields identify the connection, similar to the corresponding fields in TCP and UDP. The Source Port represents the relevant port on the endpoint that sent this packet, and the Destination Port represents the relevant port on the other endpoint. When initiating a connection, the client sHould choose its source port randomly to reduce the likelihood of attack.

DCCP APIs should treat port numbers similarly to TCP and UDP port numbers. For example, machines that distinguish between "privileged" and "unprivileged" ports for TCP and UDP should do the same for DCCP.

Data Offset: 8 bits The offset from the start of the packet's DCCP header to the start of its application data area, in 32-bit words. The receiver MUST ignore packets whose Data Offset is smaller than the minimum-sized header for the given Type or larger than the DCCP packet itself.

CCVal: 4 bits

Used by the HC-Sender CCID. For example, the A-to-B CCID'S sender, which is active at DCCP A, MAY send 4 bits of information per packet to its receiver by encoding that information in CCVal. The sender MUST set CCVal to zero unless its HC-Sender CCID specifies otherwise, and the receiver MUST ignore the CCVal field unless its HC-Receiver CCID specifies otherwise.

Checksum Coverage (CsCov): 4 bits

Checksum Coverage determines the parts of the packet that are covered by the Checksum field. This always includes the DCCP header and options, but some or all of the application data may be excluded. This can improve performance on noisy links for applications that can tolerate corruption. See Section 9.

Checksum: 16 bits

The Internet checksum of the packet's DCCP header (including options), a network-layer pseudoheader, and, depending on Checksum Coverage, all, some, or none of the application data. See section 9 .

Reserved (Res): 3 bits Senders MUST set this field to all zeroes on generated packets, and receivers MUST ignore its value. 
Type: 4 bits

The Type field specifies the type of the packet. The following values are defined:

$\begin{array}{cl}\text { Type } & \text { Meaning } \\ ---- & ------ \\ 0 & \text { DCCP-Request } \\ 1 & \text { DCCP-Response } \\ 2 & \text { DCCP-Data } \\ 3 & \text { DCCP-Ack } \\ 4 & \text { DCCP-DataAck } \\ 5 & \text { DCCP-CloseReq } \\ 6 & \text { DCCP-Close } \\ 7 & \text { DCCP-Reset } \\ 8 & \text { DCCP-Sync } \\ 9 & \text { DCCP-SyncAck } \\ 10-15 & \text { Reserved }\end{array}$

Table 1: DCCP Packet Types

Receivers MUST ignore any packets with reserved type. That is, packets with reserved type MUST NOT be processed, and they MUST NOT be acknowledged as received.

Extended Sequence Numbers (X): 1 bit

Set to one to indicate the use of an extended generic header with 48-bit Sequence and Acknowledgement Numbers. DCCP-Data, DCCPDataAck, and DCCP-Ack packets MAY set X to zero or one. All DCCP-Request, DCCP-Response, DCCP-CloseReq, DCCP-Close, DCCPReset, DCCP-Sync, and DCCP-SyncAck packets MUST set X to one; endpoints MUST ignore any such packets with X set to zero. Highrate connections SHOULD set $x$ to one on all packets to gain increased protection against wrapped sequence numbers and attacks. see section 7.6.

Sequence Number: 48 or 24 bits Identifies the packet uniquely in the sequence of all packets the source sent on this connection. Sequence Number increases by one with every packet sent, including packets such as DCCP-Ack that carry no application data. See section 7.

All currently defined packet types except DCCP-Request and DCCP-Data carry an Acknowledgement Number subheader in the four or eight bytes immediately following the generic header. When $\mathrm{x}=1$, its format is: 


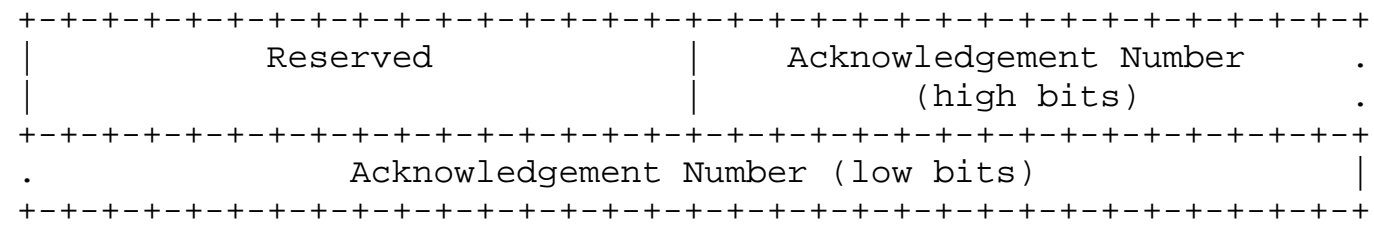

When $\mathrm{X}=0$, only the low 24 bits of the Acknowledgement Number are transmitted, giving the Acknowledgement Number Subheader this format:

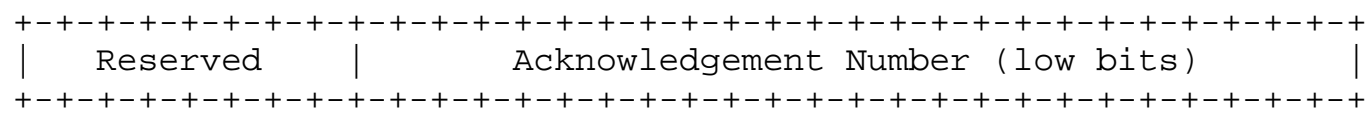

Reserved: 16 or 8 bits

Senders MUST set this field to all zeroes on generated packets, and receivers MUST ignore its value.

Acknowledgement Number: 48 or 24 bits

Generally contains GSR, the Greatest Sequence Number Received on any acknowledgeable packet so far. A packet is acknowledgeable if and only if its header was successfully processed by the receiver; Section 7.4 describes this further. Options such as Ack Vector (Section 11.4) combine with the Acknowledgement Number to provide precise information about which packets have arrived.

Acknowledgement Numbers on DCCP-Sync and DCCP-SyncAck packets need not equal GSR. See Section 5.7.

\subsection{DCCP-Request Packets}

A client initiates a DCCP connection by sending a DCCP-Request packet. These packets MAY contain application data and MUST use 48-bit sequence numbers $(X=1)$.

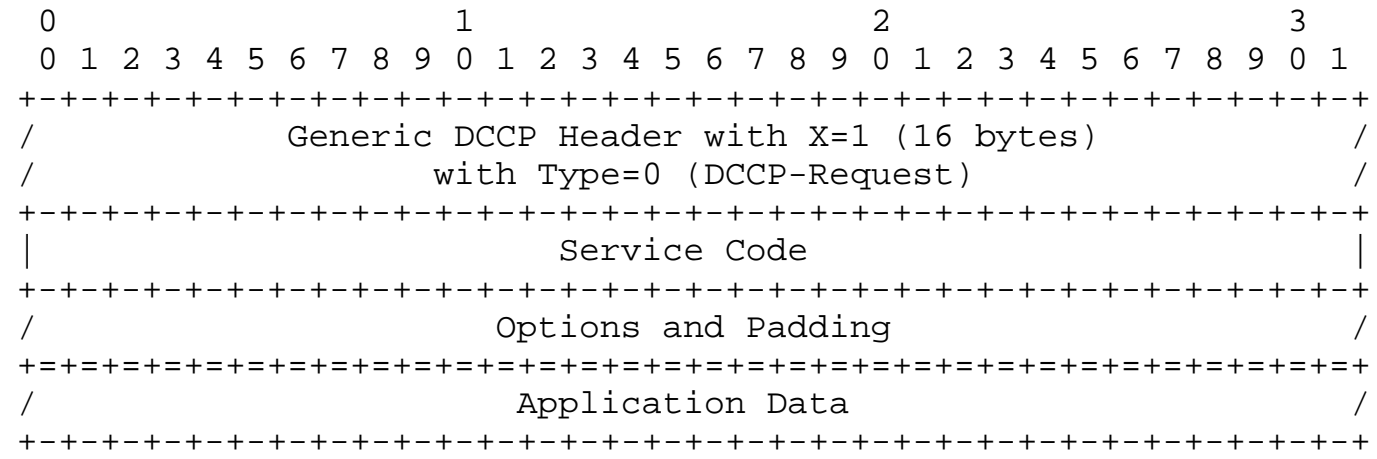


Service Code: 32 bits

Describes the application-level service to which the client application wants to connect. Service codes are intended to provide information about which application protocol a connection intends to use, thus aiding middleboxes and reducing reliance on globally well-known ports. See section 8.1.2.

5.3. DCCP-Response Packets

The server responds to valid DCCP-Request packets with DCCP-Response packets. This is the second phase of the three-way handshake. DCCP-Response packets MAY contain application data and MUST use 48-bit sequence numbers $(X=1)$.

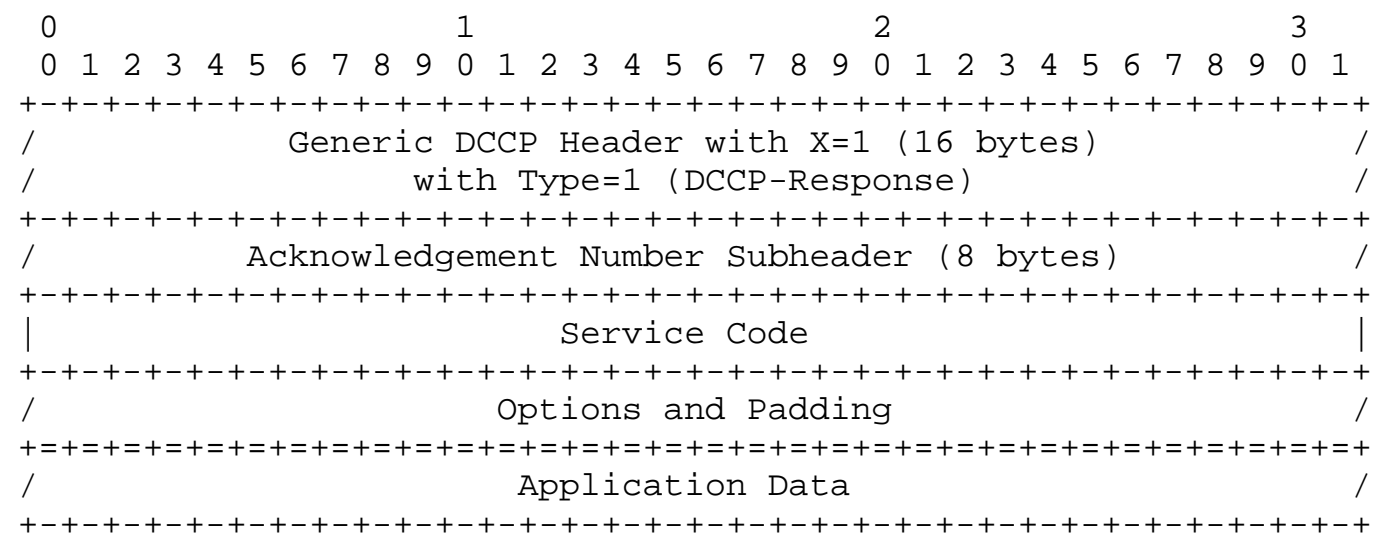

Acknowledgement Number: 48 bits

Contains GSR. Since DCCP-Responses are only sent during connection initiation, this will always equal the sequence Number on a received DCCP-Request.

Service Code: 32 bits

MUST equal the Service Code on the corresponding DCCP-Request.

5.4. DCCP-Data, DCCP-Ack, and DCCP-DataAck Packets

The central data transfer portion of every DCCP connection uses DCCP-Data, DCCP-Ack, and DCCP-DataAck packets. These packets MAY use 24-bit sequence numbers, depending on the value of the Allow Short Sequence Numbers feature (Section 7.6.1). DCCP-Data packets carry application data without acknowledgements. 


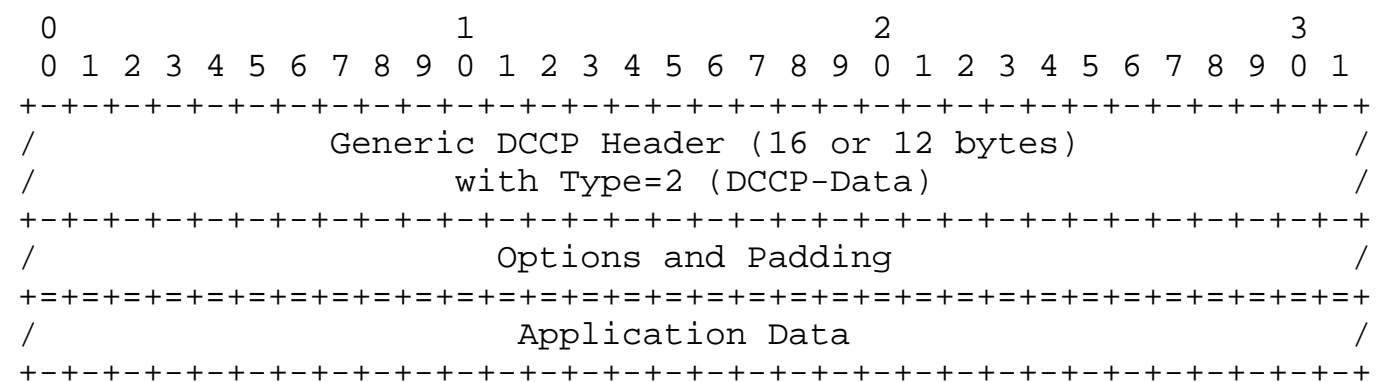

DCCP-Ack packets dispense with the data but contain an Acknowledgement Number. They are used for pure acknowledgements.

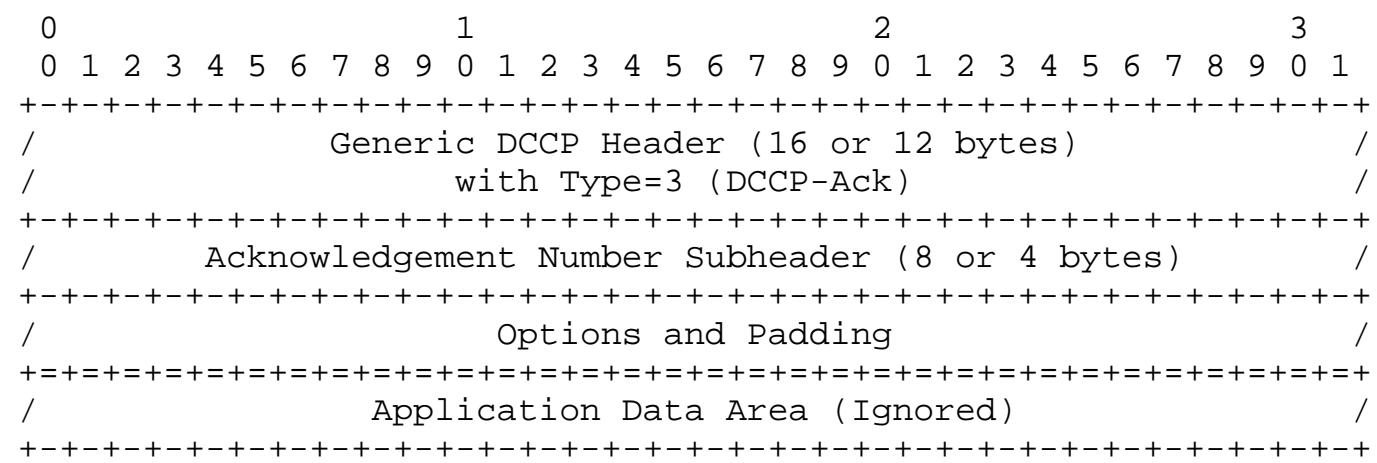

DCCP-DataAck packets carry both application data and an Acknowledgement Number. This piggybacks acknowledgement information on a data packet.

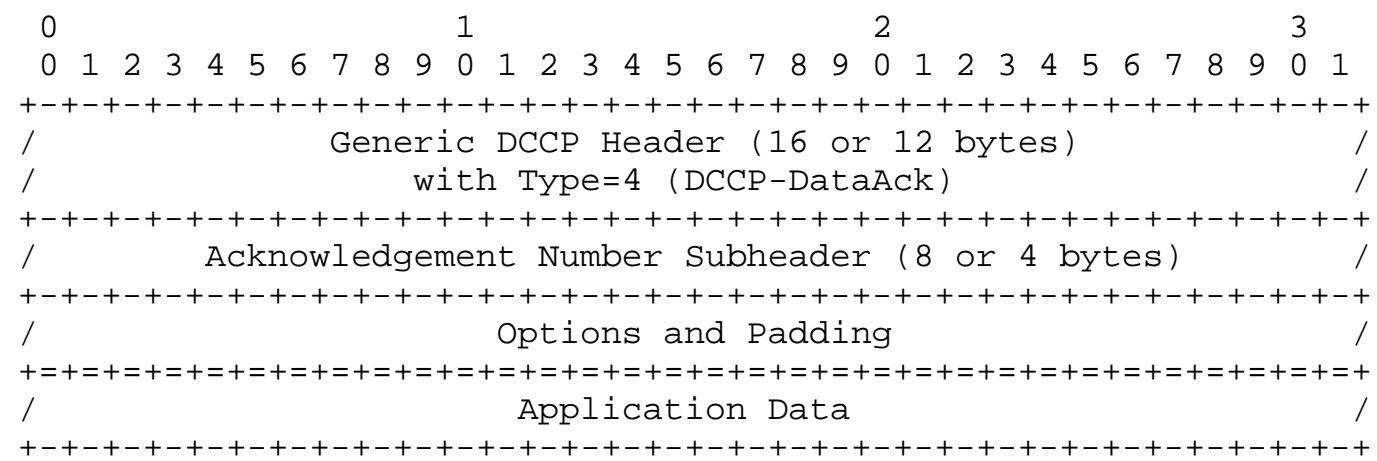

A DCCP-Data or DCCP-DataAck packet may have a zero-length application data area, which indicates that the application sent a zero-length datagram. This differs from DCCP-Request and DCCP-Response packets, where an empty application data area indicates the absence of 
application data (not the presence of zero-length application data). The API SHOULD report any received zero-length datagrams to the receiving application.

A DCCP-Ack packet MAY have a non-zero-length application data area, which essentially pads the DCCP-Ack to a desired length. Receivers MUST ignore the content of the application data area in DCCP-Ack packets.

DCCP-ACk and DCCP-DataAck packets often include additional acknowledgement options, such as Ack Vector, as required by the congestion control mechanism in use.

\subsection{DCCP-CloseReq and DCCP-Close Packets}

DCCP-CloseReq and DCCP-Close packets begin the handshake that normally terminates a connection. Either client or server may send a DCCP-Close packet, which will elicit a DCCP-Reset packet. Only the server can send a DCCP-CloseReq packet, which indicates that the server wants to close the connection but does not want to hold its TIMEWAIT state. Both packet types MUST use 48-bit sequence numbers $(\mathrm{X}=1)$.

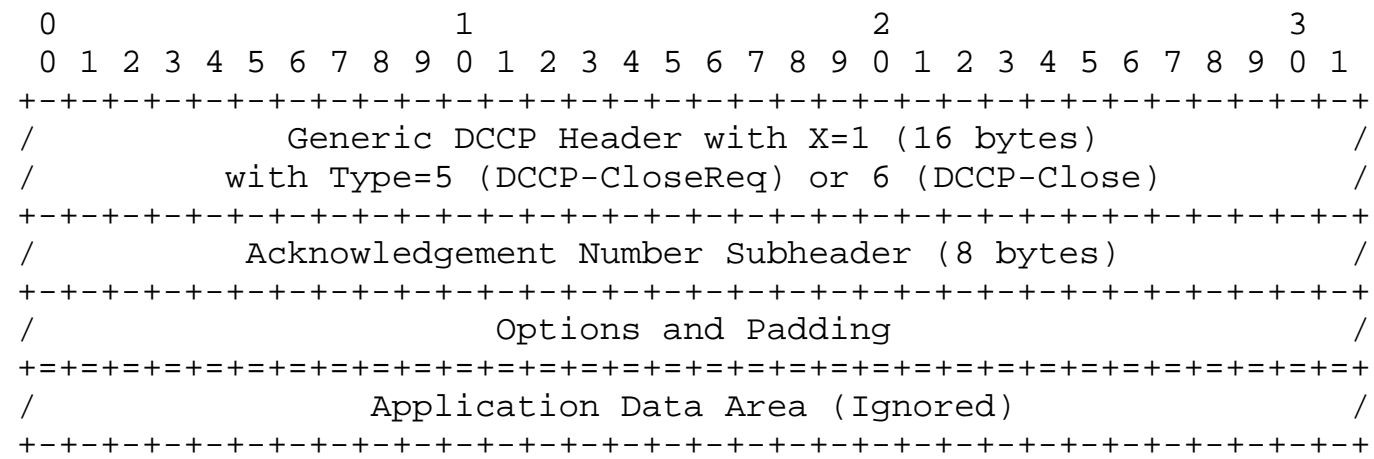

As with DCCP-Ack packets, DCCP-CloseReq and DCCP-Close packets MAY have non-zero-length application data areas, whose contents receivers MUST ignore.

\subsection{DCCP-Reset Packets}

DCCP-Reset packets unconditionally shut down a connection. Connections normally terminate with a DCCP-Reset, but resets may be sent for other reasons, including bad port numbers, bad option behavior, incorrect ECN Nonce Echoes, and so forth. DCCP-Resets MUST use 48-bit sequence numbers $(X=1)$. 


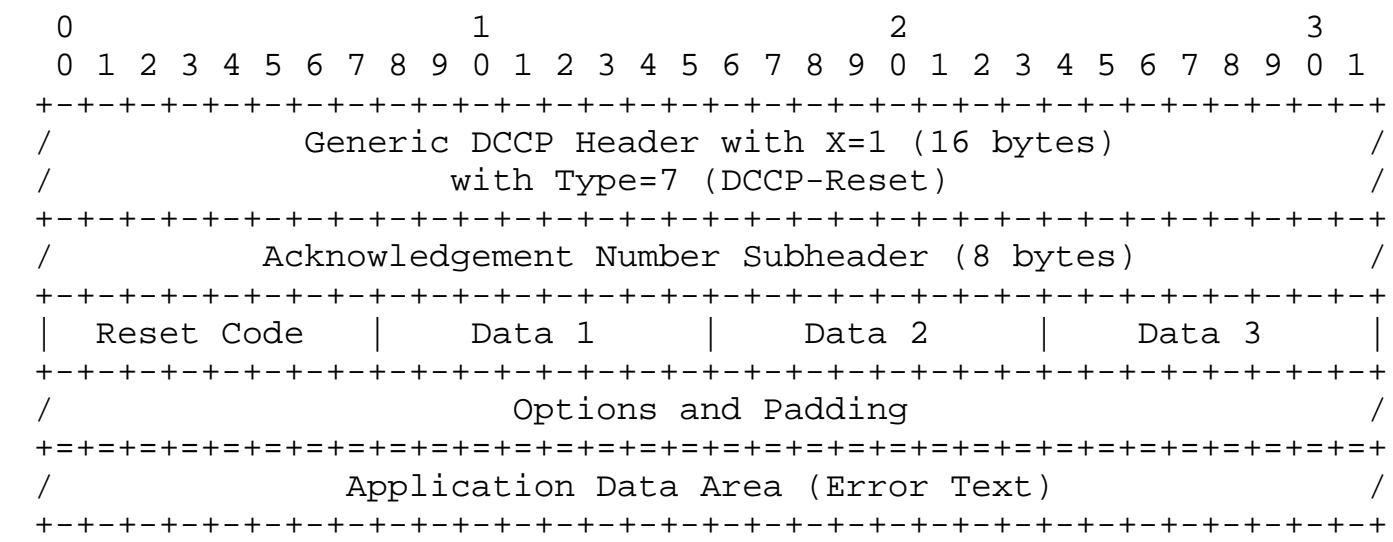

Reset Code: 8 bits

Represents the reason that the sender reset the DCCP connection.

Data 1, Data 2, and Data 3: 8 bits each

The Data fields provide additional information about why the sender reset the DCCP connection. The meanings of these fields depend on the value of Reset Code.

Application Data Area: Error Text

If present, Error Text is a human-readable text string encoded in Unicode UTF-8, and preferably in English, that describes the error in more detail. For example, a DCCP-Reset with Reset Code 11, "Aggression Penalty", might contain Error Text such as "Aggression Penalty: Received 3 bad ECN Nonce Echoes, assuming misbehavior".

The following Reset Codes are currently defined. Unless otherwise specified, the Data 1, 2, and 3 fields MUST be set to 0 by the sender of the DCCP-Reset and ignored by its receiver. Section references describe concrete situations that will cause each Reset code to be generated; they are not meant to be exhaustive.

0 , "Unspecified"

Indicates the absence of a meaningful Reset Code. Use of Reset Code 0 is NOT RECOMMENDED: the sender should choose a Reset Code that more clearly defines why the connection is being reset.

1, "Closed"

Normal connection close. See Section 8.3.

2, "Aborted"

The sending endpoint gave up on the connection because of lack of progress. See Sections 8.1.1 and 8.1.5. 
3, "No Connection"

No connection exists. See Section 8.3.1.

4, "Packet Error"

A valid packet arrived with unexpected type. For example, a DCCP-Data packet with valid header checksum and sequence numbers arrived at a connection in the REQUEST state. See Section 8.3.1. The Data 1 field equals the offending packet type as an eight-bit number; thus, an offending packet with Type 2 will result in a Data 1 value of 2 .

5, "Option Error"

An option was erroneous, and the error was serious enough to warrant resetting the connection. See Sections 6.6.7, 6.6.8, and 11.4. The Data 1 field equals the offending option type; Data 2 and Data 3 equal the first two bytes of option data (or zero if the option had less than two bytes of data).

6, "Mandatory Error"

The sending endpoint could not process an option 0 that was immediately preceded by Mandatory. The Data fields report the option type and data of option O, using the format of Reset Code 5, "Option Error". See Section 5.8.2.

7, "Connection Refused"

The Destination Port didn't correspond to a port open for listening. Sent only in response to DCCP-Requests. See Section 8.1.3.

8, "Bad Service Code"

The Service Code didn't equal the service code attached to the Destination Port. Sent only in response to DCCP-Requests. See Section 8.1 .3 .

9, "Too Busy" The server is too busy to accept new connections. Sent only in response to DCCP-Requests. See Section 8.1.3.

10, "Bad Init Cookie"

The Init Cookie echoed by the client was incorrect or missing. See Section 8.1.4.

11, "Aggression Penalty" This endpoint has detected congestion control-related misbehavior on the part of the other endpoint. See section 12.3. 
12-127, Reserved

Receivers should treat these codes as they do Reset Code 0 , "Unspecified".

128-255, CCID-specific codes Semantics depend on the connection's CCIDs. See Section 10.3. Receivers should treat unknown CCID-specific Reset Codes as they do Reset Code 0, "Unspecified".

The following table summarizes this information.

\begin{tabular}{|c|c|c|c|}
\hline $\begin{array}{l}\text { Reset } \\
\text { Code }\end{array}$ & Name & Data 1 & Data $2 \& 3$ \\
\hline----- & ---- & ------ & ---------- \\
\hline 0 & Unspecified & 0 & 0 \\
\hline 1 & Closed & 0 & 0 \\
\hline 2 & Aborted & 0 & 0 \\
\hline 3 & No Connection & 0 & 0 \\
\hline 4 & Packet Error & pkt type & 0 \\
\hline 5 & Option Error & option \# & option data \\
\hline 6 & Mandatory Error & option \# & option data \\
\hline 7 & Connection Refused & 0 & 0 \\
\hline 8 & Bad Service Code & 0 & 0 \\
\hline 9 & Too Busy & 0 & 0 \\
\hline 10 & Bad Init Cookie & 0 & 0 \\
\hline 11 & Aggression Penalty & 0 & 0 \\
\hline $12-127$ & Reserved & & \\
\hline $128-255$ & CCID-specific codes & & \\
\hline
\end{tabular}

Table 2: DCCP Reset Codes

Options on DCCP-Reset packets are processed before the connection is shut down. This means that certain combinations of options, particularly involving Mandatory, may cause an endpoint to respond to a valid DCCP-Reset with another DCCP-Reset. This cannot lead to a reset storm; since the first endpoint has already reset the connection, the second DCCP-Reset will be ignored.

5.7. DCCP-Sync and DCCP-SyncAck Packets

DCCP-Sync packets help DCCP endpoints recover synchronization after bursts of loss and recover from half-open connections. Each valid received DCCP-Sync immediately elicits a DCCP-SyncAck. Both packet types MUST use 48-bit sequence numbers $(X=1)$. 


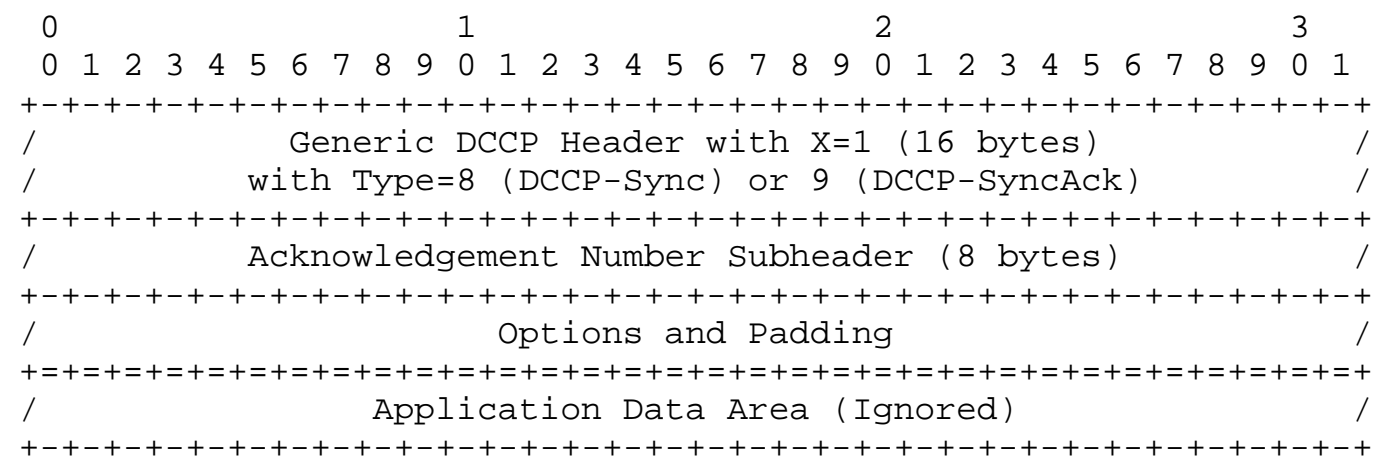

The Acknowledgement Number field has special semantics for DCCP-Sync and DCCP-SyncACk packets. First, the packet corresponding to a DCCP-Sync's Acknowledgement Number need not have been acknowledgeable. Thus, receivers MUST NOT assume that a packet was processed simply because it appears in the Acknowledgement Number field of a DCCP-Sync packet. This differs from all other packet types, where the Acknowledgement Number by definition corresponds to an acknowledgeable packet. Second, the Acknowledgement Number on any DCCP-SyncAck packet MUST correspond to the sequence Number on an acknowledgeable DCCP-Sync packet. In the presence of reordering, this might not equal GSR.

As with DCCP-Ack packets, DCCP-Sync and DCCP-SyncAck packets MAY have non-zero-length application data areas, whose contents receivers MUST ignore. Padded DCCP-Sync packets may be useful when performing Path MTU discovery; see section 14 .

\subsection{Options}

Any DCCP packet may contain options, which occupy space at the end of the DCCP header. Each option is a multiple of 8 bits in length. Individual options are not padded to multiples of 32 bits, and any option may begin on any byte boundary. However, the combination of all options MUST add up to a multiple of 32 bits; Padding options MUST be added as necessary to fill out option space to a word boundary. Any options present are included in the header checksum.

The first byte of an option is the option type. Options with types 0 through 31 are single-byte options. Other options are followed by a byte indicating the option's length. This length value includes the two bytes of option-type and option-length as well as any option-data bytes; it must therefore be greater than or equal to two.

Options MUST be processed sequentially, starting with the first option in the packet header. Options with unknown types MUST be 
ignored. Also, options with nonsensical lengths (length byte less than two or more than the remaining space in the options portion of the header) MUST be ignored, and any option space following an option with nonsensical length MUST likewise be ignored. Unless otherwise specified, multiple occurrences of the same option MUST be processed independently; for some options, this will mean in practice that the last valid occurrence of an option takes precedence.

The following options are currently defined:

\begin{tabular}{|c|c|c|c|c|}
\hline Type & $\begin{array}{l}\text { Option } \\
\text { Length }\end{array}$ & Meaning & $\begin{array}{l}\text { DCCP- } \\
\text { Data? }\end{array}$ & $\begin{array}{l}\text { Section } \\
\text { Reference }\end{array}$ \\
\hline---- & ------ & ------- & ----- & --------- \\
\hline 0 & 1 & Padding & $\mathrm{Y}$ & 5.8 .1 \\
\hline 1 & 1 & Mandatory & $\mathrm{N}$ & 5.8 .2 \\
\hline 2 & 1 & Slow Receiver & $\mathrm{Y}$ & 11.6 \\
\hline $3-31$ & 1 & Reserved & & \\
\hline 32 & variable & Change L & $\mathrm{N}$ & 6.1 \\
\hline 33 & variable & Confirm L & $\mathrm{N}$ & 6.2 \\
\hline 34 & variable & Change $\mathrm{R}$ & $\mathrm{N}$ & 6.1 \\
\hline 35 & variable & Confirm $\mathrm{R}$ & $\mathrm{N}$ & 6.2 \\
\hline 36 & variable & Init Cookie & $\mathrm{N}$ & 8.1 .4 \\
\hline 37 & $3-8$ & NDP Count & Y & 7.7 \\
\hline 38 & variable & Ack Vector [Nonce 0] & $\mathrm{N}$ & 11.4 \\
\hline 39 & variable & Ack Vector [Nonce 1] & $\mathrm{N}$ & 11.4 \\
\hline 40 & variable & Data Dropped & $\mathrm{N}$ & 11.7 \\
\hline 41 & 6 & Timestamp & Y & 13.1 \\
\hline 42 & $6 / 8 / 10$ & Timestamp Echo & Y & 13.3 \\
\hline 43 & $4 / 6$ & Elapsed Time & $\mathrm{N}$ & 13.2 \\
\hline 44 & 6 & Data Checksum & Y & 9.3 \\
\hline $45-127$ & variable & Reserved & & \\
\hline $128-255$ & variable & CCID-specific options & - & 10.3 \\
\hline
\end{tabular}

Not all options are suitable for all packet types. For example, since the Ack Vector option is interpreted relative to the Acknowledgement Number, it isn't suitable on DCCP-Request and DCCPData packets, which have no Acknowledgement Number. If an option occurs on an unexpected packet type, it MUST generally be ignored; any such restrictions are mentioned in each option's description. The table summarizes the most common restriction: when the DCCPData? column value is $\mathrm{N}$, the corresponding option MUST be ignored when received on a DCCP-Data packet. (Section 7.5.5 describes why such options are ignored as opposed to, say, causing a reset.)

Options with invalid values MUST be ignored unless otherwise specified. For example, any Data Checksum option with option length 
4 MUST be ignored, since all valid Data Checksum options have option length 6 .

This section describes two generic options, Padding and Mandatory. Other options are described later.

\subsubsection{Padding Option}

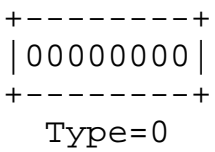

Type $=0$

Padding is a single-byte "no-operation" option used to pad between or after options. If the length of a packet's other options is not a multiple of 32 bits, then Padding options are REQUIRED to pad out the options area to the length implied by Data offset. Padding may also be used between options; for example, to align the beginning of a subsequent option on a 32-bit boundary. There is no guarantee that senders will use this option, so receivers must be prepared to process options even if they do not begin on a word boundary.

\subsubsection{Mandatory Option}

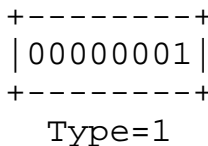

Type $=1$

Mandatory is a single-byte option that marks the immediately following option as mandatory. Say that the immediately following option is $O$. Then the Mandatory option has no effect if the receiving DCCP endpoint understands and processes $O$. If the endpoint does not understand or process 0 , however, then it MUST reset the connection using Reset Code 6, "Mandatory Failure". For instance, the endpoint would reset the connection if it did not understand $O^{\prime} \mathrm{s}$ type; if it understood $O^{\prime} s$ type, but not $O^{\prime} s$ data; if $O^{\prime} s$ data was invalid for $\mathrm{O}^{\prime} \mathrm{S}$ type; if $O$ was a feature negotiation option, and the endpoint did not understand the enclosed feature number; or if the endpoint understood $O$, but chose not to perform the action 0 implies. This list is not exhaustive and, in particular, individual option specifications may describe additional situations in which the endpoint should reset the connection and situations in which it should not.

Mandatory options MUST NOT be sent on DCCP-Data packets, and any Mandatory options received on DCCP-Data packets MUST be ignored. 
The connection is in error and should be reset with Reset Code 5, "Option Error", if option O is absent (Mandatory was the last byte of the option list), or if option O equals Mandatory. However, the combination "Mandatory Padding" is valid, and MUST behave like two bytes of Padding.

Section 6.6.9 describes the behavior of Mandatory feature negotiation options in more detail.

6. Feature Negotiation

Four DCCP options, Change L, Confirm L, Change R, and Confirm R, are used to negotiate feature values. Change options initiate a negotiation; Confirm options complete that negotiation. The "L" options are sent by the feature location, and the "R" options are sent by the feature remote. Change options are retransmitted to ensure reliability.

All these options have the same format. The first byte of option data is the feature number, and the second and subsequent data bytes hold one or more feature values. The exact format of the feature value area depends on the feature type; see section 6.3 .

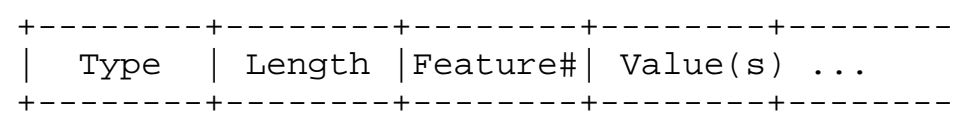

Together, the feature number and the option type ("L" or "R") uniquely identify the feature to which an option applies. The exact format of the Value(s) area depends on the feature number.

Feature negotiation options MUST NOT be sent on DCCP-Data packets, and any feature negotiation options received on DCCP-Data packets MUST be ignored.

\subsection{Change Options}

Change $L$ and Change $R$ options initiate feature negotiation. The option to use depends on the relevant feature's location: To start a negotiation for feature F/A, DCCP A will send a Change L option; to start a negotiation for $F / B$, it will send a Change $R$ option. Change options are retransmitted until some response is received. They contain at least one Value, and thus have a length of at least 4 . 


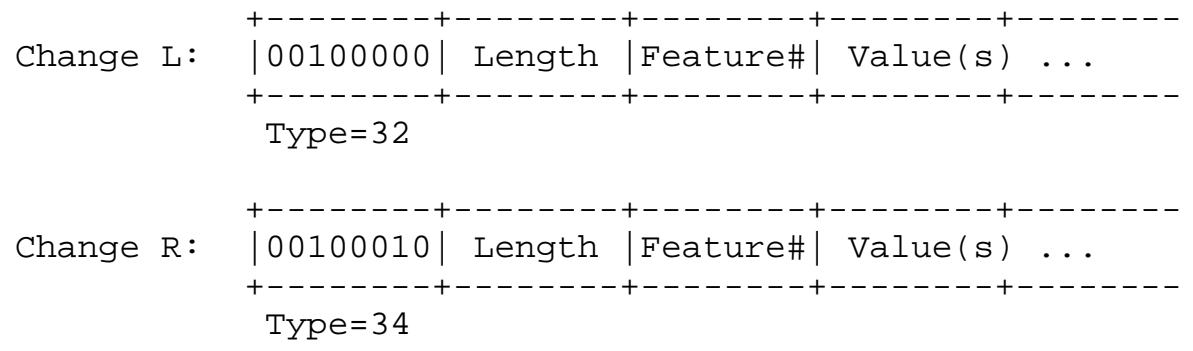

\subsection{Confirm Options}

Confirm L and Confirm $R$ options complete feature negotiation and are sent in response to Change $R$ and Change $L$ options, respectively. Confirm options MUST NOT be generated except in response to Change options. Confirm options need not be retransmitted, since Change options are retransmitted as necessary. The first byte of the Confirm option contains the feature number from the corresponding Change. Following this is the selected Value, and then possibly the sender's preference list.

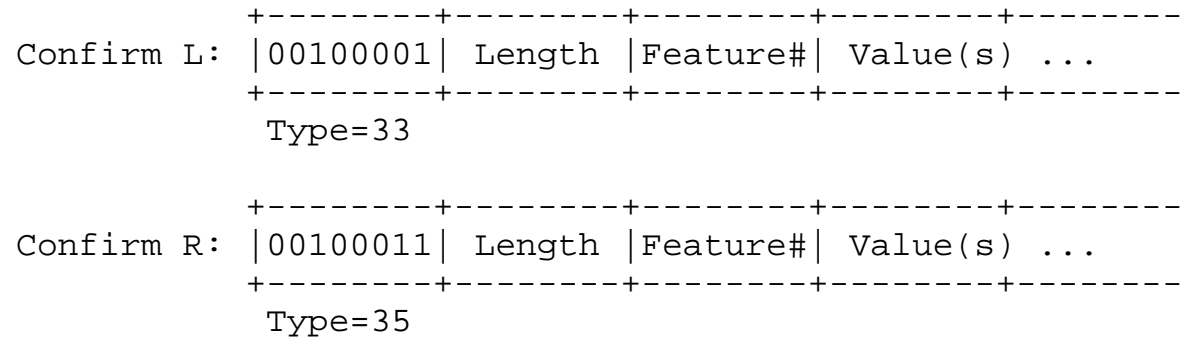

If an endpoint receives an invalid Change option -- with an unknown feature number, or an invalid value -- it will respond with an empty Confirm option containing the problematic feature number, but no value. Such options have length 3 .

\subsection{Reconciliation Rules}

Reconciliation rules determine how the two sets of preferences for a given feature are resolved into a unique result. The reconciliation rule depends only on the feature number. Each reconciliation rule must have the property that the result is uniquely determined given the contents of Change options sent by the two endpoints.

All current DCCP features use one of two reconciliation rules: server-priority ("SP") and non-negotiable ("NN"). 


\subsubsection{Server-Priority}

The feature value is a fixed-length byte string (length determined by the feature number). Each Change option contains a list of values ordered by preference, with the most preferred value coming first. Each Confirm option contains the confirmed value, followed by the confirmer's preference list. Thus, the feature's current value will generally appear twice in Confirm options' data, once as the current value and once in the confirmer's preference list.

To reconcile the preference lists, select the first entry in the server's list that also occurs in the client's list. If there is no shared entry, the feature's value MUST NOT change, and the Confirm option will confirm the feature's previous value (unless the Change option was Mandatory; see Section 6.6.9).

\subsubsection{Non-Negotiable}

The feature value is a byte string. Each option contains exactly one feature value. The feature location signals a new value by sending a Change L option. The feature remote MUST accept any valid value, responding with a Confirm $R$ option containing the new value, and it MUST send empty Confirm $R$ options in response to invalid values (unless the Change L option was Mandatory; see Section 6.6.9). Change $R$ and Confirm L options MUST NOT be sent for non-negotiable features; see Section 6.6.8. Non-negotiable features use the feature negotiation mechanism to achieve reliability. 
6.4. Feature Numbers

This document defines the following feature numbers.

\begin{tabular}{|c|c|c|c|c|c|}
\hline Number & Meaning & $\begin{array}{l}\text { Rec'n } \\
\text { Rule }\end{array}$ & $\begin{array}{c}\text { Initial } \\
\text { Value }\end{array}$ & $\operatorname{Req}^{\prime} \mathrm{d}$ & $\begin{array}{c}\text { Section } \\
\text { Reference }\end{array}$ \\
\hline------ & ------- & ----- & ----- & ----- & \\
\hline 0 & Reserved & & & & \\
\hline 1 & Congestion Control ID (CCID) & SP & 2 & $\mathrm{Y}$ & 10 \\
\hline 2 & Allow Short Seqnos & $\mathrm{SP}$ & 0 & Y & 7.6 .1 \\
\hline 3 & Sequence Window & $\mathrm{NN}$ & 100 & $\mathrm{Y}$ & 7.5 .2 \\
\hline 4 & ECN Incapable & SP & 0 & $\mathrm{~N}$ & 12.1 \\
\hline 5 & Ack Ratio & $\mathrm{NN}$ & 2 & $\mathrm{~N}$ & 11.3 \\
\hline 6 & Send Ack Vector & $\mathrm{SP}$ & 0 & $\mathrm{~N}$ & 11.5 \\
\hline 7 & Send NDP Count & $\mathrm{SP}$ & 0 & $\mathrm{~N}$ & 7.7 .2 \\
\hline 8 & Minimum Checksum Coverage & $\mathrm{SP}$ & 0 & $\mathrm{~N}$ & 9.2 .1 \\
\hline 9 & Check Data Checksum & $\mathrm{SP}$ & 0 & $\mathrm{~N}$ & 9.3 .1 \\
\hline $10-127$ & Reserved & & & & \\
\hline $128-255$ & CCID-specific features & & & & 10.3 \\
\hline
\end{tabular}

Table 4: DCCP Feature Numbers

Rec'n Rule The reconciliation rule used for the feature. SP means server-priority, NN means non-negotiable.

Initial Value The initial value for the feature. Every feature has a known initial value.

Req'd This column is "Y" if and only if every DCCP implementation MUST understand the feature. If it is "N", then the feature behaves like an extension (see Section 15), and it is safe to respond to Change options for the feature with empty Confirm options. Of course, a CCID might require the feature; a DCCP that implements CCID 2 MUST support Ack Ratio and Send Ack Vector, for example. 


\subsection{Feature Negotiation Examples}

Here are three example feature negotiations for features located at the server, the first two for the Congestion Control ID feature, the last for the Ack Ratio.

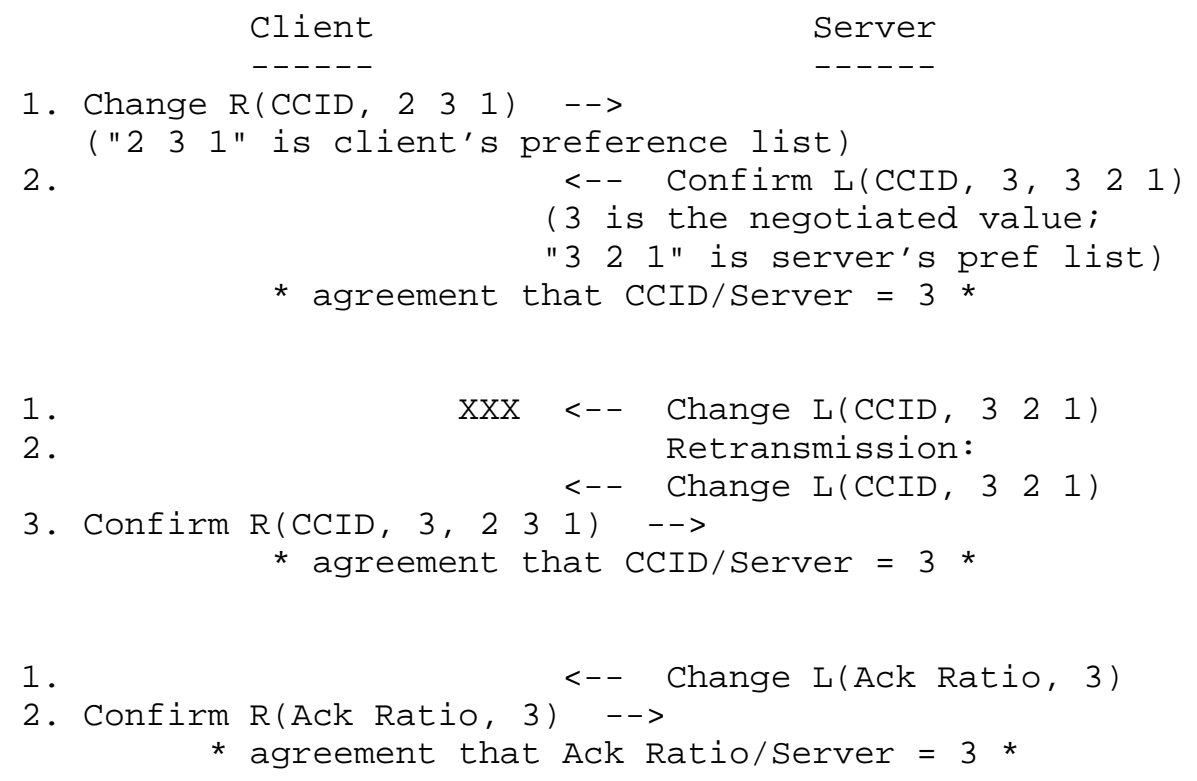


Change L(Sequence Window, 1024) $=32,9,3,0,0,0,0,4,0$ DCCP B should change Sequence Window/A's value (feature number 3, a non-negotiable feature) to the 6-byte string $0,0,0,0,4,0$ (the value 1024).

Confirm L(CCID, 2, 2 3) $=33,6,1,2,2,3$ DCCP A has changed CCID/A's value to 2 ; its preferred values are 2 and 3 , in that preference order.

Empty Confirm L $(126)=33,3,126$ DCCP A doesn't implement feature number 126, or DCCP B's proposed value for feature $126 /$ A was invalid.

Change $\mathrm{R}(\mathrm{CCID}, 32)=34,5,1,3,2$ DCCP B should change CCID/B's value; DCCP A's preferred values are 3 and 2 , in that preference order.

Confirm R(CCID, 2, 32 2) $=35,6,1,2,3,2$ $\mathrm{DCCP} A$ has changed CCID/B's value to 2 ; its preferred values were 3 and 2 , in that preference order.

Confirm $R$ (Sequence Window, 1024) $=35,9,3,0,0,0,0,4,0$ DCCP A has changed Sequence Window/B's value to the 6-byte string $0,0,0,0,4,0$ (the value 1024).

Empty Confirm $R(126)=35,3,126$ DCCP A doesn't implement feature number 126, or DCCP B's proposed value for feature 126/B was invalid.

6.6. Option Exchange

A few basic rules govern feature negotiation option exchange.

1. Every non-reordered Change option gets a Confirm option in response.

2. Change options are retransmitted until a response for the latest Change is received.

3. Feature negotiation options are processed in strictly-increasing order by Sequence Number.

The rest of this section describes the consequences of these rules in more detail. 


\subsubsection{Normal Exchange}

Change options are generated when a DCCP endpoint wants to change the value of some feature. Generally, this will happen at the beginning of a connection, although it may happen at any time. We say the endpoint "generates" or "sends" a Change L or Change R option, but of course the option must be attached to a packet. The endpoint may attach the option to a packet it would have generated anyway (such as a DCCP-Request), or it may create a "feature negotiation packet", often a DCCP-Ack or DCCP-Sync, just to carry the option. Feature negotiation packets are controlled by the relevant congestion control mechanism. For example, DCCP A may send a DCCP-Ack or DCCP-Sync for feature negotiation only if the B-to-A CCID would allow sending a DCCP-Ack. In addition, an endpoint SHOULD generate at most one feature negotiation packet per round-trip time.

On receiving a Change $L$ or Change $\mathrm{R}$ option, a DCCP endpoint examines the included preference list, reconciles that with its own preference list, calculates the new value, and sends back a Confirm $\mathrm{R}$ or Confirm L option, respectively, informing its peer of the new value or that the feature was not understood. Every non-reordered Change option MUST result in a corresponding Confirm option, and any packet including a Confirm option MUST carry an Acknowledgement Number. (Section 6.6.4 describes how Change reordering is detected and handled.) Generated Confirm options may be attached to packets that would have been sent anyway (such as DCCP-Response or DCCP-SyncACk) or to new feature negotiation packets, as described above.

The Change-sending endpoint MUST wait to receive a corresponding Confirm option before changing its stored feature value. The Confirm-sending endpoint changes its stored feature value as soon as it sends the Confirm.

A packet MAY contain more than one feature negotiation option, possibly including two options that refer to the same feature; as usual, the options are processed sequentially.

\subsubsection{Processing Received Options}

DCCP endpoints exist in one of three states relative to each feature. STABLE is the normal state, where the endpoint knows the feature's value and thinks the other endpoint agrees. An endpoint enters the CHANGING state when it first sends a Change for the feature and returns to STABLE once it receives a corresponding confirm. The final state, UNSTABLE, indicates that an endpoint in CHANGING state changed its preference list but has not yet transmitted a Change option with the new preference list. 
Feature state transitions at a feature location are implemented according to this diagram. The diagram ignores sequence number and option validity issues; these are handled explicitly in the pseudocode that follows.
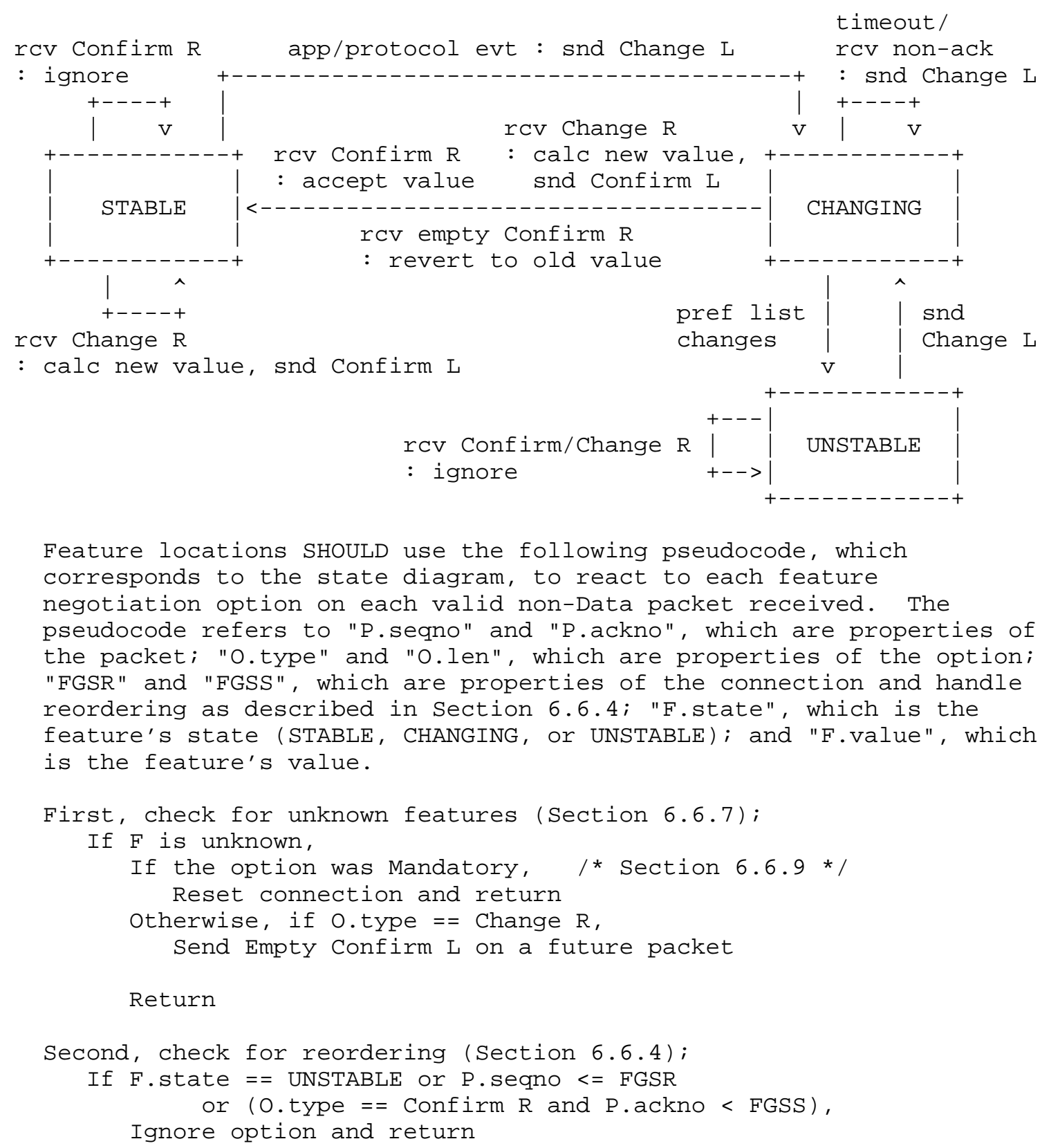


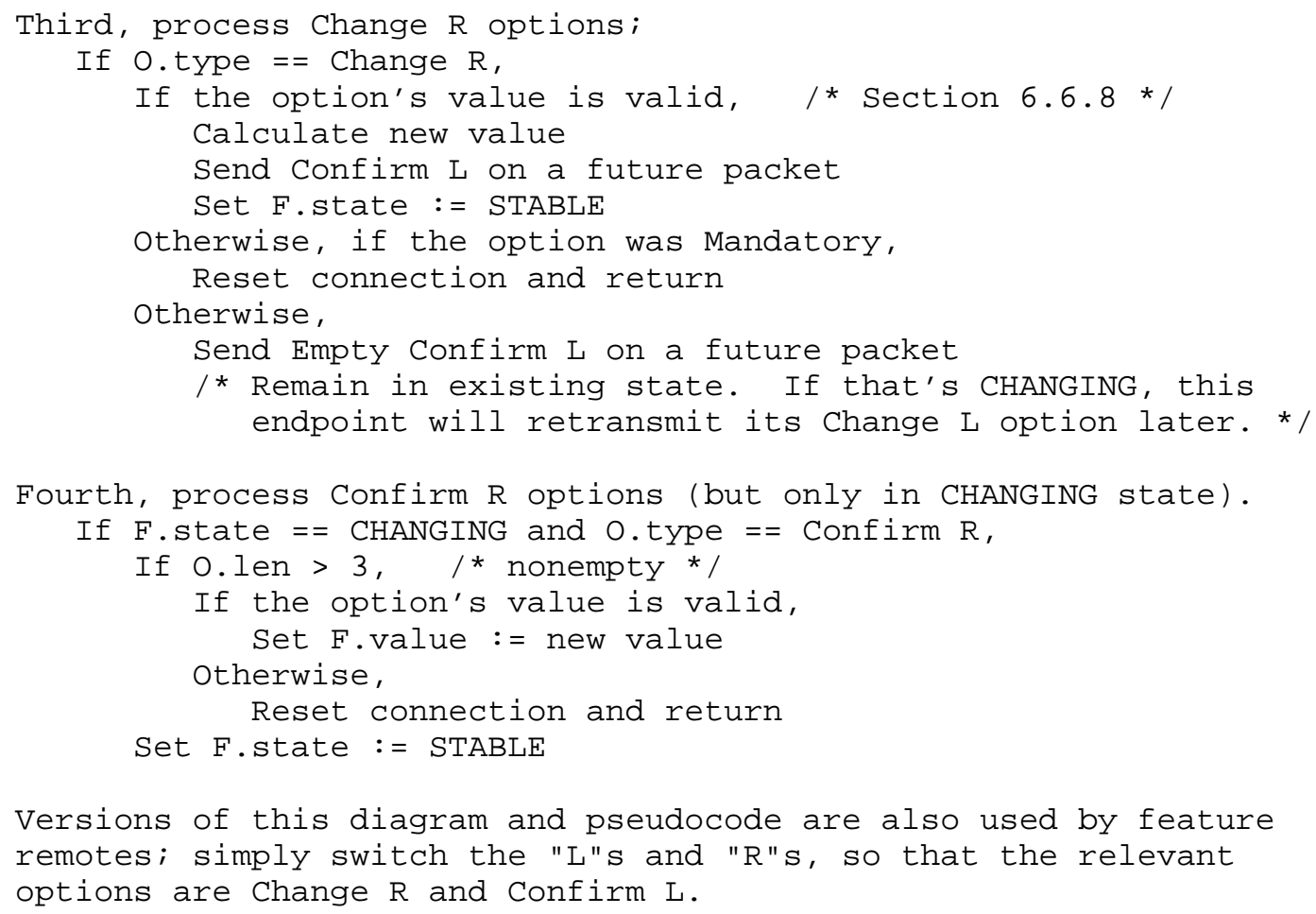

6.6.3. Loss and Retransmission

Packets containing Change and Confirm options might be lost or delayed by the network. Therefore, Change options are repeatedly transmitted to achieve reliability. We refer to this as "retransmission", although of course there are no packet-level retransmissions in DCCP: a Change option that is sent again will be sent on a new packet with a new sequence number.

A CHANGING endpoint transmits another Change option once it realizes that it has not heard back from the other endpoint. The new Change option need not contain the same payload as the original; reordering protection will ensure that agreement is reached based on the most recently transmitted option.

A CHANGING endpoint MUST continue retransmitting Change options until it gets some response or the connection terminates.

Endpoints SHOULD use an exponential-backoff timer to decide when to retransmit Change options. (Packets generated specifically for feature negotiation MUST use such a timer.) The timer interval is initially set to not less than one round-trip time, and should back 
off to not less than 64 seconds. The backoff protects against delayed agreement due to the reordering protection algorithms described in the next section. Again, endpoints may piggyback change options on packets they would have sent anyway or create new packets to carry the options. Any new packets are controlled by the relevant congestion-control mechanism.

Confirm options are never retransmitted, but the Confirm-sending endpoint MUST generate a Confirm option after every non-reordered Change.

\subsubsection{Reordering}

Reordering might cause packets containing Change and Confirm options to arrive in an unexpected order. Endpoints MUST ignore feature negotiation options that do not arrive in strictly-increasing order by Sequence Number. The rest of this section presents two algorithms that fulfill this requirement.

The first algorithm introduces two sequence number variables that each endpoint maintains for the connection.

FGSR Feature Greatest Sequence Number Received: The greatest sequence number received, considering only valid packets that contained one or more feature negotiation options (Change and/or Confirm). This value is initialized to ISR - 1 .

FGSS Feature Greatest Sequence Number sent: The greatest sequence number sent, considering only packets that contained one or more new Change options. A Change option is new if and only if it was generated during a transition from the STABLE or UNSTABLE state to the CHANGING state; Change options generated within the CHANGING state are retransmissions and MUST have exactly the same contents as previously transmitted options, allowing tolerance for reordering. FGSS is initialized to ISS.

Each endpoint checks two conditions on sequence numbers to decide whether to process received feature negotiation options.

1. If a packet's sequence Number is less than or equal to FGSR, then its Change options MUST be ignored.

2. If a packet's sequence Number is less than or equal to FGSR, if it has no Acknowledgement Number, OR if its Acknowledgement Number is less than FGSS, then its Confirm options MUST be ignored. 
Alternatively, an endpoint MAY maintain separate FGSR and FGSS values for every feature. FGSR (F/X) would equal the greatest sequence number received, considering only packets that contained Change or Confirm options applying to feature F/X; FGSS(F/X) would be defined similarly. This algorithm requires more state, but is slightly more forgiving to multiple overlapped feature negotiations. Either algorithm MAY be used; the first algorithm, with connection-wide FGSR and FGSS variables, is RECOMMENDED.

One consequence of these rules is that a CHANGING endpoint will ignore any Confirm option that does not acknowledge the latest Change option sent. This ensures that agreement, once achieved, used the most recent available information about the endpoints' preferences.

\subsubsection{Preference Changes}

Endpoints are allowed to change their preference lists at any time. However, an endpoint that changes its preference list while in the CHANGING state MUST transition to the UNSTABLE state. It will transition back to CHANGING once it has transmitted a Change option with the new preference list. This ensures that agreement is based on active preference lists. Without the UNSTABLE state, simultaneous negotiation -- where the endpoints began independent negotiations for the same feature at the same time -- might lead to the negotiation's terminating with the endpoints thinking the feature had different values.

6.6.6. Simultaneous Negotiation

The two endpoints might simultaneously open negotiation for the same feature, after which an endpoint in the CHANGING state will receive a Change option for the same feature. Such received Change options can act as responses to the original Change options. The CHANGING endpoint MUST examine the received Change's preference list, reconcile that with its own preference list (as expressed in its generated Change options), and generate the corresponding Confirm option. It can then transition to the STABLE state. 


\subsubsection{Unknown Features}

Endpoints may receive Change options referring to feature numbers they do not understand -- for instance, when an extended DCCP converses with a non-extended DCCP. Endpoints MUST respond to unknown Change options with Empty Confirm options (that is, Confirm options containing no data), which inform the CHANGING endpoint that the feature was not understood. However, if the Change option was Mandatory, the connection MUST be reset; see Section 6.6.9.

On receiving an empty Confirm option for some feature, the CHANGING endpoint MUST transition back to the STABLE state, leaving the feature's value unchanged. Section 15 suggests that the default value for any extension feature correspond to "extension not available".

Some features are required to be understood by all DCCPs (see Section 6.4). The CHANGING endpoint SHOULD reset the connection (with Reset Code 5, "Option Error") if it receives an empty Confirm option for such a feature.

Since Confirm options are generated only in response to Change options, an endpoint should never receive a Confirm option referring to a feature number it does not understand. Nevertheless, endpoints MUST ignore any such options they receive.

\subsubsection{Invalid Options}

A DCCP endpoint might receive a Change or Confirm option for a known feature that lists one or more values that it does not understand. Some, but not all, such options are invalid, depending on the relevant reconciliation rule (Section 6.3). For instance:

- All features have length limitations, and options with invalid lengths are invalid. For example, the Ack Ratio feature takes 16-bit values, so valid "Confirm R(Ack Ratio)" options have option length 5 .

- Some non-negotiable features have value limitations. The Ack Ratio feature takes two-byte, non-zero integer values, so a "Change L(Ack Ratio, 0)" option is never valid. Note that server-priority features do not have value limitations, since unknown values are handled as a matter of course.

- Any Confirm option that selects the wrong value, based on the two preference lists and the relevant reconciliation rule, is invalid. 
However, unexpected Confirm options -- that refer to unknown feature numbers, or that don't appear to be part of a current negotiation -are not invalid, although they are ignored by the receiver.

An endpoint receiving an invalid Change option MUST respond with the corresponding empty Confirm option. An endpoint receiving an invalid Confirm option MUST reset the connection, with Reset Code 5, "Option Error".

\subsubsection{Mandatory Feature Negotiation}

Change options may be preceded by Mandatory options (Section 5.8.2). Mandatory Change options are processed like normal Change options except that the following failure cases will cause the receiver to reset the connection with Reset Code 6, "Mandatory Failure", rather than send a Confirm option. The connection MUST be reset if:

o the Change option's feature number was not understood;

- the Change option's value was invalid, and the receiver would normally have sent an empty Confirm option in response; or

o for server-priority features, there was no shared entry in the two endpoints' preference lists.

Other failure cases do not cause connection reset; in particular, reordering protection may cause a Mandatory Change option to be ignored without resetting the connection.

Confirm options behave identically and have the same reset conditions whether or not they are Mandatory.

7. Sequence Numbers

DCCP uses sequence numbers to arrange packets into sequence, to detect losses and network duplicates, and to protect against attackers, half-open connections, and the delivery of very old packets. Every packet carries a sequence Number; most packet types carry an Acknowledgement Number as well.

DCCP sequence numbers are packet based. That is, sequence Numbers generated by each endpoint increase by one, modulo $2^{\wedge} 48$, per packet. Even DCCP-Ack and DCCP-Sync packets, and other packets that don't carry user data, increment the sequence Number. Since DCCP is an unreliable protocol, there are no true retransmissions, but effective retransmissions, such as retransmissions of DCCP-Request packets, also increment the sequence Number. This lets DCCP implementations 
detect network duplication, retransmissions, and acknowledgement loss; it is a significant departure from TCP practice.

\subsection{Variables}

DCCP endpoints maintain a set of sequence number variables for each connection.

ISS The Initial sequence Number sent by this endpoint. This equals the Sequence Number of the first DCCP-Request or DCCP-Response sent.

ISR The Initial Sequence Number Received from the other endpoint. This equals the sequence Number of the first DCCP-Request or DCCP-Response received.

GSS The Greatest Sequence Number Sent by this endpoint. Here, and elsewhere, "greatest" is measured in circular sequence space.

GSR The Greatest Sequence Number Received from the other endpoint on an acknowledgeable packet. (Section 7.4 defines this term.)

GAR The Greatest Acknowledgement Number Received from the other endpoint on an acknowledgeable packet that was not a DCCPSync.

Some other variables are derived from these primitives.

SWL and SWH

(Sequence Number Window Low and High) The extremes of the validity window for received packets' Sequence Numbers.

AWL and AWH

(Acknowledgement Number Window Low and High) The extremes of the validity window for received packets' Acknowledgement Numbers .

\subsection{Initial Sequence Numbers}

The endpoints' initial sequence numbers are set by the first DCCPRequest and DCCP-Response packets sent. Initial sequence numbers MUST be chosen to avoid two problems:

- delivery of old packets, where packets lingering in the network from an old connection are delivered to a new connection with the same addresses and port numbers; and 
- sequence number attacks, where an attacker can guess the sequence numbers that a future connection would use [M85].

These problems are the same as those faced by TCP, and DCCP implementations SHOULD use TCP's strategies to avoid them [RFC793, RFC1948]. The rest of this section explains these strategies in more detail.

To address the first problem, an implementation MUST ensure that the initial sequence number for a given <source address, source port, destination address, destination port> 4-tuple doesn't overlap with recent sequence numbers on previous connections with the same 4-tuple. ("Recent" means sent within 2 maximum segment lifetimes, or 4 minutes.) The implementation MUST additionally ensure that the lower 24 bits of the initial sequence number don't overlap with the lower 24 bits of recent sequence numbers (unless the implementation plans to avoid short sequence numbers; see Section 7.6). An implementation that has state for a recent connection with the same 4-tuple can pick a good initial sequence number explicitly. Otherwise, it could tie initial sequence number selection to some clock, such as the 4-microsecond clock used by TCP [RFC793]. Two separate clocks may be required, one for the upper 24 bits and one for the lower 24 bits.

To address the second problem, an implementation MUST provide each 4-tuple with an independent initial sequence number space. Then, opening a connection doesn't provide any information about initial sequence numbers on other connections to the same host. [RFC1948] achieves this by adding a cryptographic hash of the 4-tuple and a secret to each initial sequence number. For the secret, [RFC1948] recommends a combination of some truly random data [RFC4086], an administratively installed passphrase, the endpoint's IP address, and the endpoint's boot time, but truly random data is sufficient. Care should be taken when the secret is changed; such a change alters all initial sequence number spaces, which might make an initial sequence number for some 4-tuple equal a recently sent sequence number for the same 4-tuple. To avoid this problem, the endpoint might remember dead connection state for each 4-tuple or stay quiet for 2 maximum segment lifetimes around such a change.

\subsection{Quiet Time}

DCCP endpoints, like TCP endpoints, must take care before initiating connections when they boot. In particular, they MUST NOT send packets whose sequence numbers are close to the sequence numbers of packets lingering in the network from before the boot. The simplest way to enforce this rule is for DCCP endpoints to avoid sending any packets until one maximum segment lifetime (2 minutes) after boot. 
Other enforcement mechanisms include remembering recent sequence numbers across boots and reserving the upper 8 or so bits of initial sequence numbers for a persistent counter that decrements by two each boot. (The latter mechanism would require disallowing packets with short sequence numbers; see Section 7.6.1.)

7.4. Acknowledgement Numbers

Cumulative acknowledgements are meaningless in an unreliable protocol. Therefore, DCCP's Acknowledgement Number field has a different meaning from $\mathrm{TCP}^{\prime} \mathrm{s}$.

A received packet is classified as acknowledgeable if and only if its header was successfully processed by the receiving DCCP. In terms of the pseudocode in Section 8.5, a received packet becomes acknowledgeable when the receiving endpoint reaches step 8 . This means, for example, that all acknowledgeable packets have valid header checksums and sequence numbers. A sent packet's Acknowledgement Number MUST equal the sending endpoint's GSR, the Greatest Sequence Number Received on an acknowledgeable packet, for all packet types except DCCP-Sync and DCCP-SyncAck.

"Acknowledgeable" does not refer to data processing. Even acknowledgeable packets may have their application data dropped, due to receive buffer overflow or corruption, for instance. Data Dropped options report these data losses when necessary, letting congestion control mechanisms distinguish between network losses and endpoint losses. This issue is discussed further in sections 11.4 and 11.7.

DCCP-Sync and DCCP-SyncAck packets' Acknowledgement Numbers differ as follows: The Acknowledgement Number on a DCCP-Sync packet corresponds to a received packet, but not necessarily to an acknowledgeable packet; in particular, it might correspond to an out-of-sync packet whose options were not processed. The Acknowledgement Number on a DCCP-SyncAck packet always corresponds to an acknowledgeable DCCPSync packet; it might be less than GSR in the presence of reordering.

\subsection{Validity and Synchronization}

Any DCCP endpoint might receive packets that are not actually part of the current connection. For instance, the network might deliver an old packet, an attacker might attempt to hijack a connection, or the other endpoint might crash, causing a half-open connection.

DCCP, like TCP, uses sequence number checks to detect these cases. Packets whose Sequence and/or Acknowledgement Numbers are out of range are called sequence-invalid and are not processed normally. 
Unlike TCP, DCCP requires a synchronization mechanism to recover from large bursts of loss. One endpoint might send so many packets during a burst of loss that when one of its packets finally got through, the other endpoint would label its sequence Number as invalid. A handshake of DCCP-Sync and DCCP-SyncAck packets recovers from this case.

\subsubsection{Sequence and Acknowledgement Number Windows}

Each DCCP endpoint defines sequence validity windows that are subsets of the Sequence and Acknowledgement Number spaces. These windows correspond to packets the endpoint expects to receive in the next few round-trip times. The Sequence and Acknowledgement Number windows always contain GSR and GSS, respectively. The window widths are controlled by Sequence Window features for the two half-connections.

The Sequence Number validity window for packets from DCCP B is [SWL, SWH]. This window always contains GSR, the Greatest sequence Number Received on a sequence-valid packet from DCCP B. It is W packets wide, where $W$ is the value of the Sequence Window/B feature. Onefourth of the sequence window, rounded down, is less than or equal to GSR, and three-fourths is greater than GSR. (This asymmetric placement assumes that bursts of loss are more common in the network than significant reorderings.)

\begin{tabular}{|c|c|c|c|}
\hline $\begin{array}{c}\text { invalid } \\
<--------*\end{array}$ & 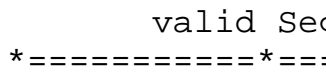 & $\begin{array}{l}\operatorname{rs} \\
========\text { * }\end{array}$ & $\begin{array}{c}\text { invalid } \\
\star--------\end{array}$ \\
\hline $\begin{array}{r}\text { GSR - } \\
\text { floor }(W / 4)\end{array}$ & $\begin{array}{l}\text { GSR }+1-\text { GSR } \\
\text { floor }(W / 4)\end{array}$ & $\begin{array}{r}\text { GSR + } \\
\operatorname{ceil}(3 W / 4)\end{array}$ & $\begin{array}{l}\text { GSR }+1+ \\
\operatorname{ceil}(3 W / 4)\end{array}$ \\
\hline
\end{tabular}

The Acknowledgement Number validity window for packets from DCCP B is $[A W L, A W H]$. The high end of the window, AWH, equals GSS, the Greatest Sequence Number Sent by DCCP A; the window is $W^{\prime}$ packets wide, where $W^{\prime}$ is the value of the sequence Window/A feature.

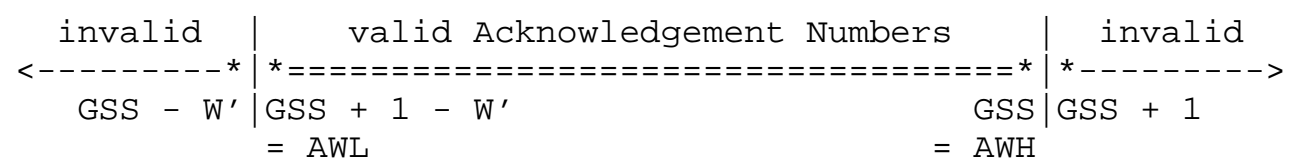

SWL and AWL are initially adjusted so that they are not less than the initial sequence Numbers received and sent, respectively:

$$
\begin{aligned}
& \mathrm{SWL}:=\max (\mathrm{GSR}+1-\mathrm{floor}(\mathrm{W} / 4), \mathrm{ISR}), \\
& \mathrm{AWL}:=\max \left(\mathrm{GSS}+1-\mathrm{W}^{\prime}, \mathrm{ISS}\right) .
\end{aligned}
$$


These adjustments MUST be applied only at the beginning of the connection. (Long-lived connections may wrap sequence numbers so that they appear to be less than ISR or ISS; the adjustments MUST NOT be applied in that case.)

\subsubsection{Sequence Window Feature}

The Sequence Window/A feature determines the width of the Sequence Number validity window used by DCCP B and the width of the Acknowledgement Number validity window used by DCCP A. DCCP A sends a "Change L (Sequence Window, W)" option to notify DCCP B that the Sequence Window/A value is W.

Sequence Window has feature number 3 and is non-negotiable. It takes 48-bit (6-byte) integer values, like DCCP sequence numbers. Change and Confirm options for Sequence Window are therefore 9 bytes long. New connections start with sequence Window 100 for both endpoints. The minimum valid Sequence Window value is Wmin $=32$. The maximum valid Sequence Window value is Wmax $=2^{\wedge} 46-1=70368744177663$. Change options suggesting sequence Window values out of this range are invalid and MUST be handled accordingly.

A proper Sequence Window/A value must reflect the number of packets DCCP A expects to be in flight. Only DCCP A can anticipate this number. Values that are too small increase the risk of the endpoints getting out sync after bursts of loss, and values that are much too small can prevent productive communication whether or not there is loss. On the other hand, too-large values increase the risk of connection hijacking; Section 7.5.5 quantifies this risk. One good guideline is for each endpoint to set sequence Window to about five times the maximum number of packets it expects to send in a roundtrip time. Endpoints SHOULD send Change L(Sequence Window) options, as necessary, as the connection progresses. Also, an endpoint MUST NOT persistently send more than its Sequence Window number of packets per round-trip time; that is, DCCP A MUST NOT persistently send more than Sequence Window/A packets per RTT.

\subsubsection{Sequence-Validity Rules}

Sequence-validity depends on the received packet's type. This table shows the sequence and acknowledgement number checks applied to each packet; a packet is sequence-valid if it passes both tests, and sequence-invalid if it does not. Many of the checks refer to the sequence and acknowledgement number validity windows [SWL, SWH] and $[A W L, A W H]$ defined in Section 7.5.1. 


Packet Type
---------
DCCP-Request
DCCP-Response
DCCP-Data
DCCP-Ack
DCCP-DataAck
DCCP-CloseReq
DCCP-Close
DCCP-Reset
DCCP-Sync
DCCP-SyncAck

Sequence Number Check
$-----------=$ seqno $<=$ SWH $\left.\quad{ }^{*}\right)$
SWL $<=$ s
SWL $<=$ seqno $<=$ SWH $\left.\quad{ }^{*}\right)$
SWL $<=$ seqno $<=$ SWH
SWL $<=$ seqno $<=$ SWH
SWL $<=$ seqno $<=$ SWH
GSR $<$ seqno $<=$ SWH
GSR $<$ seqno $<=$ SWH
GSR $<$ seqno $<=$ SWH
SWL $<=$ seqno
SWL $<=$ seqno

Acknowledgement Number Check

$\mathrm{N} / \mathrm{A}$
$\mathrm{AWL}<=$ ackno $<=$ AWH
$\mathrm{N} / \mathrm{A}$
AWL $<=$ ackno $<=$ AWH
AWL $<=$ ackno $<=$ AWH
GAR $<=$ ackno $<=$ AWH
GAR $<=$ ackno $<=$ AWH
GAR $<=$ ackno $<=$ AWH
AWL $<=$ ackno $<=$ AWH
AWL $<=$ ackno $<=$ AWH

(*) Check not applied if connection is in LISTEN or REQUEST state.

In general, packets are sequence-valid if their sequence and Acknowledgement Numbers lie within the corresponding valid windows, $[S W L, S W H]$ and $[A W L, A W H]$. The exceptions to this rule are as follows:

- Since DCCP-CloseReq, DCCP-Close, and DCCP-Reset packets end a connection, they cannot have sequence Numbers less than or equal to GSR, or Acknowledgement Numbers less than GAR.

- DCCP-Sync and DCCP-SyncAck Sequence Numbers are not strongly checked. These packet types exist specifically to get the endpoints back into sync; checking their sequence Numbers would eliminate their usefulness.

The lenient checks on DCCP-Sync and DCCP-SyncAck packets allow continued operation after unusual events, such as endpoint crashes and large bursts of loss, but there's no need for leniency in the absence of unusual events -- that is, during ongoing successful communication. Therefore, DCCP implementations SHOULD use the following, more stringent checks for active connections, where a connection is considered active if it has received valid packets from the other endpoint within the last three round-trip times.

\begin{tabular}{|c|c|c|}
\hline Packet Type & Sequence Number Check & $\begin{array}{l}\text { Acknowledgement Number } \\
\text { Check } \\
--------------------\end{array}$ \\
\hline DCCP-Sync & SWL $<=$ seqno $<=$ SWH & AWL $<=$ ackno $<=$ AWH \\
\hline DCCP-SyncAck & SWL $<=$ seqno $<=$ SWH & AWL $<=$ ackno $<=$ AWH \\
\hline
\end{tabular}


Since these checks can cause extra synchronization overhead and delay connection closing when packets are lost, they should be considered experimental.

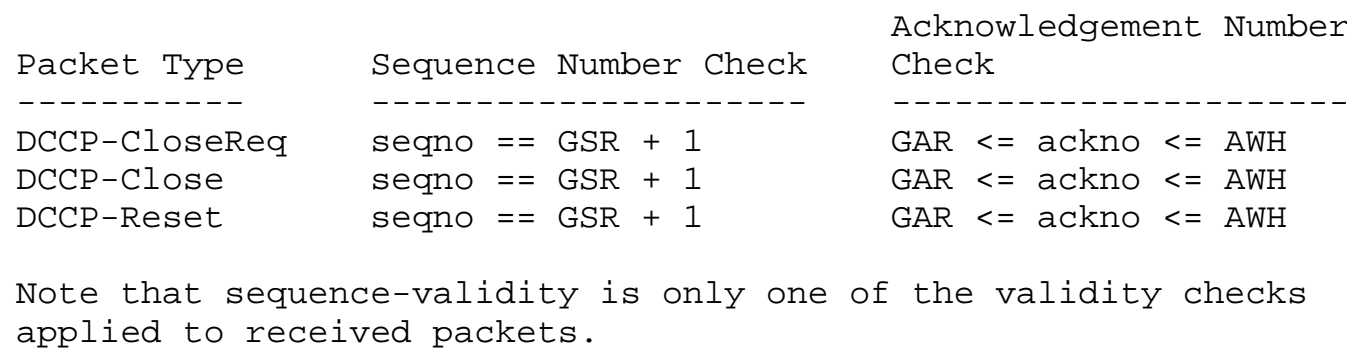

7.5.4. Handling Sequence-Invalid Packets

Endpoints respond to received sequence-invalid packets as follows.

- Any sequence-invalid DCCP-Sync or DCCP-SyncAck packet MUST be ignored.

- A sequence-invalid DCCP-Reset packet MUST elicit a DCCP-SynC packet in response (subject to a possible rate limit). This response packet MUST use a new Sequence Number, and thus will increase GSS; GSR will not change, however, since the received packet was sequence-invalid. The response packet's Acknowledgement Number MUST equal GSR.

- Any other sequence-invalid packet MUST elicit a similar DCCP-Sync packet, except that the response packet's Acknowledgement Number MUST equal the sequence-invalid packet's sequence Number.

On receiving a sequence-valid DCCP-Sync packet, the peer endpoint (say, DCCP B) MUST update its GSR variable and reply with a DCCPSyncAck packet. The DCCP-SyncACk packet's Acknowledgement Number will equal the DCCP-Sync's Sequence Number, which is not necessarily GSR. Upon receiving this DCCP-SyncAck, which will be sequence-valid since it acknowledges the DCCP-Sync, DCCP A will update its GSR variable, and the endpoints will be back in sync. As an exception, if the peer endpoint is in the REQUEST state, it MUST respond with a DCCP-Reset instead of a DCCP-SyncACk. This serves to clean up DCCP $A^{\prime} \mathrm{s}$ half-open connection.

To protect against denial-of-service attacks, DCCP implementations SHOULD impose a rate limit on DCCP-Syncs sent in response to sequence-invalid packets, such as not more than eight DCCP-Syncs per second. 
DCCP endpoints MUST NOT process sequence-invalid packets except, perhaps, by generating a DCCP-Sync. For instance, options MUST NOT be processed. An endpoint MAY temporarily preserve sequence-invalid packets in case they become valid later, however; this can reduce the impact of bursts of loss by delivering more packets to the application. In particular, an endpoint MAY preserve sequenceinvalid packets for up to 2 round-trip times. If, within that time, the relevant sequence windows change so that the packets become sequence-valid, the endpoint MAY process them again.

Note that sequence-invalid DCCP-Reset packets cause DCCP-Syncs to be generated. This is because endpoints in an unsynchronized state (CLOSED, REQUEST, and LISTEN) might not have enough information to generate a proper DCCP-Reset on the first try. For example, if a peer endpoint is in CLOSED state and receives a DCCP-Data packet, it cannot guess the right sequence Number to use on the DCCP-Reset it generates (since the DCCP-Data packet has no Acknowledgement Number). The DCCP-Sync generated in response to this bad reset serves as a challenge, and contains enough information for the peer to generate a proper DCCP-Reset. However, the new DCCP-Reset may carry a different Reset Code than the original DCCP-Reset; probably the new Reset Code will be 3, "No Connection". The endpoint SHOULD use information from the original DCCP-Reset when possible.

\subsubsection{Sequence Number Attacks}

Sequence and Acknowledgement Numbers form DCCP's main line of defense against attackers. An attacker that cannot guess sequence numbers cannot easily manipulate or hijack a DCCP connection, and requirements like careful initial sequence number choice eliminate the most serious attacks.

An attacker might still send many packets with randomly chosen Sequence and Acknowledgement Numbers, however. If one of those probes ends up sequence-valid, it may shut down the connection or otherwise cause problems. The easiest such attacks to execute are as follows:

- Send DCCP-Data packets with random Sequence Numbers. If one of these packets hits the valid sequence number window, the attack packet's application data may be inserted into the data stream.

- Send DCCP-Sync packets with random Sequence and Acknowledgement Numbers. If one of these packets hits the valid acknowledgement number window, the receiver will shift its sequence number window accordingly, getting out of sync with the correct endpoint -perhaps permanently. 
The attacker has to guess both Source and Destination Ports for any of these attacks to succeed. Additionally, the connection would have to be inactive for the DCCP-Sync attack to succeed, assuming the victim implemented the more stringent checks for active connections recommended in section 7.5 .3 .

To quantify the probability of success, let $\mathrm{N}$ be the number of attack packets the attacker is willing to send, $W$ be the relevant sequence window width, and $\mathrm{L}$ be the length of sequence numbers (24 or 48). The attacker's best strategy is to space the attack packets evenly over sequence space. Then the probability of hitting one sequence number window is $\mathrm{P}=\mathrm{WN} / 2^{\wedge} \mathrm{L}$.

The success probability for a DCCP-Data attack using short sequence numbers thus equals $\mathrm{P}=\mathrm{WN} / 2^{\wedge} 24$. For $\mathrm{W}=100$, then, the attacker must send more than 83,000 packets to achieve a 50\% chance of success. For reference, the easiest TCP attack -- sending a SYN with a random sequence number, which will cause a connection reset if it falls within the window - - with $\mathrm{W}=8760$ (a common default) and $\mathrm{L}=32$ requires more than 245,000 packets to achieve a 50\% chance of success.

A fast connection's W will generally be high, increasing the attack success probability for fixed N. If this probability gets uncomfortably high with $\mathrm{L}=24$, the endpoint SHOULD prevent the use of short sequence numbers by manipulating the Allow short sequence Numbers feature (see Section 7.6.1). The probability limit depends on the application, however. Some applications, such as those already designed to handle corruption, are quite resilient to data injection attacks.

The DCCP-Sync attack has $\mathrm{L}=48$, since DCCP-Sync packets use long sequence numbers exclusively; in addition, the success probability is halved, since only half the Sequence Number space is valid. Attacks have a correspondingly smaller probability of success. For a large $W$ of 2000 packets, then, the attacker must send more than 10^11 packets to achieve a $50 \%$ chance of success.

Attacks involving DCCP-Ack, DCCP-DataAck, DCCP-CloseReq, DCCP-Close, and DCCP-Reset packets are more difficult, since sequence and Acknowledgement Numbers must both be guessed. The probability of attack success for these packet types equals $\mathrm{P}=\mathrm{WXN} / \mathrm{2}^{\wedge}(2 \mathrm{~L})$, where $\mathrm{W}$ is the Sequence Number window, $\mathrm{X}$ is the Acknowledgement Number window, and $\mathrm{N}$ and $\mathrm{L}$ are as before.

Since DCCP-Data attacks with short sequence numbers are relatively easy for attackers to execute, DCCP has been engineered to prevent these attacks from escalating to connection resets or other serious 
7.6. Short Sequence Numbers

DCCP sequence numbers are 48 bits long. This large sequence space protects DCCP connections against some blind attacks, such as the injection of DCCP-Resets into the connection. However, DCCP-Data, DCCP-Ack, and DCCP-DataAck packets, which make up the body of any DCCP connection, may reduce header space by transmitting only the lower 24 bits of the relevant Sequence and Acknowledgement Numbers. The receiving endpoint will extend these numbers to 48 bits using the following pseudocode:

procedure Extend_Sequence_Number ( $S, \mathrm{REF}$ )

I* $S$ is a 24-bit sequence number from the packet header. REF is the relevant 48-bit reference sequence number:

GSS if $S$ is an Acknowledgement Number, and GSR if $S$ is a Sequence Number. */

Set REF_low $:=$ low 24 bits of REF

Set REF_hi $:=$ high 24 bits of REF

If $\mathrm{REF}$ _low $(<) \mathrm{S} \quad /$ circular comparison mod $2^{\wedge} 24$ * and $S|<|$ REF_low, /* conventional, non-circular comparison */

Return ( ( REF_hi + 1) mod 2^24) $<<24) \mid \mathrm{S}$

Otherwise, if $\mathrm{S}(<) \mathrm{REF}$ _low and $\mathrm{REF}$ llow $|<| \mathrm{S}$, Return $\left(\left((\right.\right.$ REF_hi -1$\left.\left.) \bmod 2^{\wedge} 24\right)<<24\right) \mid S$

Otherwise, Return (REF_hi $<24) \mid$ S

The two different kinds of comparison in the if statements detect when the low-order bits of the sequence space have wrapped. (The circular comparison "REF_low $(<)$ S" returns true if and only if (S - REF_low), calculated using two's-complement arithmetic and then represented as an unsigned number, is less than or equal to $2^{\wedge} 23$ (mod 2^24).) When this happens, the high-order bits are incremented or decremented, as appropriate.

7.6.1. Allow Short Sequence Numbers Feature

Endpoints can require that all packets use long sequence numbers by leaving the Allow Short sequence Numbers feature value at its default of zero. This can reduce the risk that data will be inappropriately injected into the connection. DCCP A sends a "Change L (Allow Short Seqnos, 1)" option to indicate its desire to send packets with short sequence numbers.

Allow Short Sequence Numbers has feature number 2 and is serverpriority. It takes one-byte Boolean values. When Allow Short Seqnos/B is zero, DCCP B MUST NOT send packets with short sequence numbers and DCCP A MUST ignore any packets with short sequence 
numbers that are received. Values of two or more are reserved. New connections start with Allow Short sequence Numbers 0 for both endpoints.

\subsubsection{When to Avoid Short Sequence Numbers}

Short sequence numbers reduce the rate DCCP connections can safely achieve and increase the risks of certain kinds of attacks, including blind data injection. Very-high-rate DCCP connections, and connections with large sequence windows (Section 7.5.2), SHOULD NOT use short sequence numbers on their data packets. The attack risk issues have been discussed in section 7.5.5; we discuss the rate limitation issue here.

The sequence-validity mechanism assumes that the network does not deliver extremely old data. In particular, it assumes that the network must have dropped any packet by the time the connection wraps around and uses its sequence number again. This constraint limits the maximum connection rate that can be safely achieved. Let MSL equal the maximum segment lifetime, $\mathrm{P}$ equal the average DCCP packet size in bits, and L equal the length of sequence numbers (24 or 48 bits). Then the maximum safe rate, in bits per second, is $\mathrm{R}=\mathrm{P}^{*}\left(2^{\wedge} \mathrm{L}\right) / 2 \mathrm{MSL}$.

For the default MSL of 2 minutes, 1500-byte DCCP packets, and short sequence numbers, the safe rate is therefore approximately $800 \mathrm{Mb} / \mathrm{s}$. Although 2 minutes is a very large MSL for any networks that could sustain that rate with such small packets, long sequence numbers allow much higher rates under the same constraints: up to 14 petabits a second for 1500-byte packets and the default MSL.

7.7. NDP Count and Detecting Application Loss

DCCP's sequence numbers increment by one on every packet, including non-data packets (packets that don't carry application data). This makes DCCP sequence numbers suitable for detecting any network loss, but not for detecting the loss of application data. The NDP Count option reports the length of each burst of non-data packets. This lets the receiving DCCP reliably determine when a burst of loss included application data.

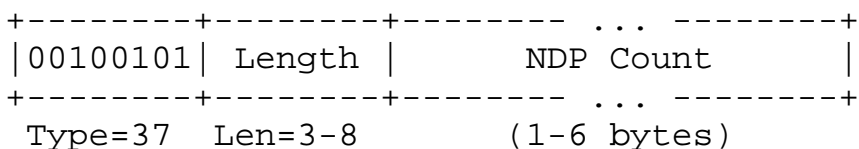

If a DCCP endpoint's send NDP Count feature is one (see below), then that endpoint MUST send an NDP Count option on every packet whose 
immediate predecessor was a non-data packet. Non-data packets consist of DCCP packet types DCCP-Ack, DCCP-Close, DCCP-CloseReq, DCCP-Reset, DCCP-Sync, and DCCP-SyncAck. The other packet types, namely DCCP-Request, DCCP-Response, DCCP-Data, and DCCP-DataAck, are considered data packets, although not all DCCP-Request and DCCPResponse packets will actually carry application data.

The value stored in NDP Count equals the number of consecutive nondata packets in the run immediately previous to the current packet. Packets with no NDP Count option are considered to have NDP Count zero.

The NDP Count option can carry one to six bytes of data. The smallest option format that can hold the NDP Count SHOULD be used.

With NDP Count, the receiver can reliably tell only whether a burst of loss contained at least one data packet. For example, the receiver cannot always tell whether a burst of loss contained a nondata packet.

\subsubsection{NDP Count Usage Notes}

Say that $K$ consecutive sequence numbers are missing in some burst of loss, and that the send NDP Count feature is on. Then some application data was lost within those sequence numbers unless the packet following the hole contains an NDP Count option whose value is greater than or equal to $\mathrm{K}$.

For example, say that an endpoint sent the following sequence of non-data packets $(\mathrm{Nx})$ and data packets $(\mathrm{Dx})$.

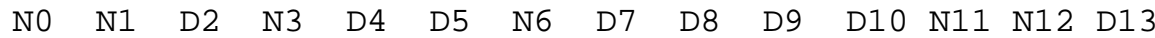

Those packets would have NDP Counts as follows.

$\begin{array}{llllllllllllll}\text { N0 } & \text { N1 } & \text { D2 } & \text { N3 } & \text { D4 } & \text { D5 } & \text { N6 } & \text { D7 } & \text { D8 } & \text { D9 } & \text { D10 } & \text { N11 } & \text { N12 } & \text { D13 } \\ - & 1 & 2 & - & 1 & - & - & 1 & - & - & - & - & 1 & 2\end{array}$

NDP Count is not useful for applications that include their own sequence numbers with their packet headers.

\subsubsection{Send NDP Count Feature}

The Send NDP Count feature lets DCCP endpoints negotiate whether they should send NDP Count options on their packets. DCCP A sends a "Change R(Send NDP Count, 1)" option to ask DCCP B to send NDP Count options. 
Send NDP Count has feature number 7 and is server-priority. It takes one-byte Boolean values. DCCP B MUST send NDP Count options as described above when send NDP Count/B is one, although it MAY send NDP Count options even when Send NDP Count/B is zero. Values of two or more are reserved. New connections start with send NDP Count 0 for both endpoints.

8. Event Processing

This section describes how DCCP connections move between states and which packets are sent when. Note that feature negotiation takes place in parallel with the connection-wide state transitions described here.

\subsection{Connection Establishment}

DCCP connections' initiation phase consists of a three-way handshake: an initial DCCP-Request packet sent by the client, a DCCP-Response sent by the server in reply, and finally an acknowledgement from the client, usually via a DCCP-Ack or DCCP-DataAck packet. The client moves from the REQUEST state to PARTOPEN, and finally to OPEN; the server moves from LISTEN to RESPOND, and finally to OPEN.

\begin{tabular}{|c|c|c|c|c|c|}
\hline \multirow{2}{*}{\multicolumn{2}{|c|}{$\begin{array}{c}\text { Client State } \\
\text { CLOSED }\end{array}$}} & & & \multicolumn{2}{|c|}{ Server Stat } \\
\hline & & & & & LISTEN \\
\hline 1 . & REQUEST & $-->$ & Request & $-->$ & \\
\hline 2 . & & $<--$ & Response & $<--$ & RESPOND \\
\hline 3. & PARTOPEN & $-->$ & Ack, DataAck & $-->$ & \\
\hline 4 . & & $<--$ & Data, Ack, DataAck & $<--$ & OPEN \\
\hline 5 . & OPEN & $<->$ & Data, Ack, DataAck & $<->$ & OPEN \\
\hline
\end{tabular}

\subsubsection{Client Request}

When a client decides to initiate a connection, it enters the REQUEST state, chooses an initial sequence number (Section 7.2), and sends a $\mathrm{DCCP}-$ Request packet using that sequence number to the intended server.

DCCP-Request packets will commonly carry feature negotiation options that open negotiations for various connection parameters, such as preferred congestion control IDs for each half-connection. They may also carry application data, but the client should be aware that the server may not accept such data.

A client in the REQUEST state SHOULD use an exponential-backoff timer to send new DCCP-Request packets if no response is received. The first retransmission should occur after approximately one second, backing off to not less than one packet every 64 seconds; or the 
endpoint can use whatever retransmission strategy is followed for retransmitting TCP SYNs. Each new DCCP-Request MUST increment the Sequence Number by one and MUST contain the same Service Code and application data as the original DCCP-Request.

A client MAY give up on its DCCP-Requests after some time (3 minutes, for example). When it does, it SHOULD send a DCCP-Reset packet to the server with Reset Code 2, "Aborted", to clean up state in case one or more of the Requests actually arrived. A client in REQUEST state has never received an initial sequence number from its peer, so the DCCP-Reset's Acknowledgement Number MUST be set to zero.

The client leaves the REQUEST state for PARTOPEN when it receives a DCCP-Response from the server.

\subsubsection{Service Codes}

Each DCCP-Request contains a 32-bit Service Code, which identifies the application-level service to which the client application is trying to connect. Service codes should correspond to application services and protocols. For example, there might be a Service Code for SIP control connections and one for RTP audio connections. Middleboxes, such as firewalls, can use the Service code to identify the application running on a nonstandard port (assuming the DCCP header has not been encrypted).

Endpoints MUST associate a Service Code with every DCCP socket, both actively and passively opened. The application will generally supply this Service Code. Each active socket MUST have exactly one Service Code. Passive sockets MAY, at the implementation's discretion, be associated with more than one Service Code; this might let multiple applications, or multiple versions of the same application, listen on the same port, differentiated by Service Code. If the DCCP-Request's Service Code doesn't equal any of the server's Service Codes for the given port, the server MUST reject the request by sending a DCCPReset packet with Reset Code 8, "Bad Service Code". A middlebox MAY also send such a DCCP-Reset in response to packets whose Service Code is considered unsuitable.

Service Codes are not intended to be DCCP-specific and are allocated by IANA. Following the policies outlined in [RFC2434], most Service Codes are allocated First Come First Served, subject to the following guidelines.

- Service Codes are allocated one at a time, or in small blocks. A short English description of the intended service is REQUIRED to obtain a Service Code assignment, but no specification, standards 
track or otherwise, is necessary. IANA maintains an association of Service Codes to the corresponding phrases.

- Users request specific Service Code values. We suggest that users request Service Codes that can be represented using the "SC:" formatting convention described below. Thus, the "Frobodyne Plotz Protocol" might correspond to Service Code 17178548426 or, equivalently, "SC:fdpz". The canonical interpretation of a Service Code field is numeric.

- Service codes whose bytes each have values in the set $\{32,45-57$, 65-90\} use a specification Required allocation policy. That is, these Service Codes are used for international standard or standards-track specifications, IETF or otherwise. (This set consists of the ASCII digits, uppercase letters, and characters space, ' $-',{ }^{\prime} \cdot{ }^{\prime}$, and ' $/$ '.)

- Service Codes whose high-order byte equals 63 (ASCII '?') are reserved for Private Use.

- Service Code 0 represents the absence of a meaningful Service Code and MUST NOT be allocated.

- The value 4294967295 is an invalid Service Code. Servers MUST reject any DCCP-Request with this Service Code value by sending a DCCP-Reset packet with Reset Code 8, "Bad Service Code".

This design for Service Code allocation is based on the allocation of 4-byte identifiers for Macintosh resources, PNG chunks, and TrueType and Opentype tables.

In text settings, we recommend that Service codes be written in one of three forms, prefixed by the ASCII letters SC and either a colon ":" or equals sign "=". These forms are interpreted as follows.

SC: Indicates a Service Code representable using a subset of the ASCII characters. The colon is followed by one to four characters taken from the following set: letters, digits, and the characters in "-_+.*/? @" (not including quotes). Numerically, these characters have values in $\{42-43,45-57$, 63-90, 95, 97-122\}. The Service code is calculated by padding the string on the right with spaces (value 32) and intepreting the four-character result as a 32-bit big-endian number.

$\mathrm{SC}=\quad$ Indicates a decimal service Code. The equals sign is followed by any number of decimal digits, which specify the Service Code. Values above 4294967294 are illegal. 


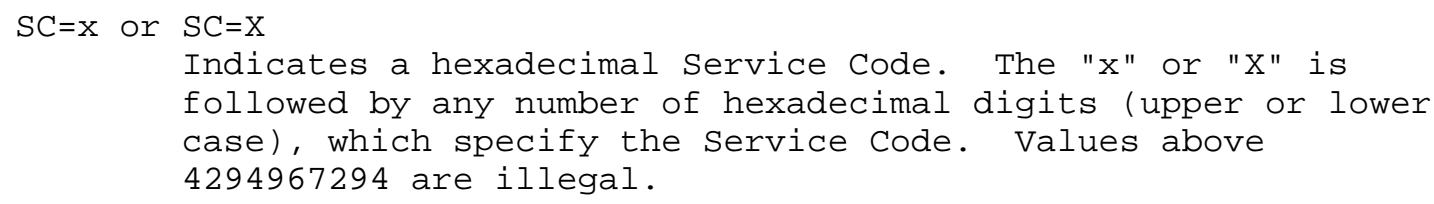

Thus, the Service Code 1717858426 might be represented in text as either $S C: f d p z, S C=1717858426$, or $S C=x 6664707 \mathrm{~A}$.

\subsubsection{Server Response}

In the second phase of the three-way handshake, the server moves from the LISTEN state to RESPOND and sends a DCCP-Response message to the client. In this phase, a server will often specify the features it would like to use, either from among those the client requested or in addition to those. Among these options is the congestion control mechanism the server expects to use.

The server MAY respond to a DCCP-Request packet with a DCCP-Reset packet to refuse the connection. Relevant Reset Codes for refusing a connection include 7, "Connection Refused", when the DCCP-Request's Destination Port did not correspond to a DCCP port open for listening; 8, "Bad Service Code", when the DCCP-Request's Service Code did not correspond to the service code registered with the Destination Port; and 9, "Too Busy", when the server is currently too busy to respond to requests. The server SHOULD limit the rate at which it generates these resets; for example, to not more than 1024 per second.

The server SHOULD NOT retransmit DCCP-Response packets; the client will retransmit the $\mathrm{DCCP}$-Request if necessary. (Note that the "retransmitted" DCCP-Request will have, at least, a different sequence number from the "original" DCCP-Request. The server can thus distinguish true retransmissions from network duplicates.) The server will detect that the retransmitted DCCP-Request applies to an existing connection because of its Source and Destination Ports. Every valid DCCP-Request received while the server is in the RESPOND state MUST elicit a new DCCP-Response. Each new DCCP-Response MUST increment the server's Sequence Number by one and MUST include the same application data, if any, as the original DCCP-Response.

The server MUST NOT accept more than one piece of DCCP-Request application data per connection. In particular, the DCCP-Response sent in reply to a retransmitted DCCP-Request with application data SHOULD contain a Data Dropped option, in which the retransmitted DCCP-Request data is reported with Drop Code 0, Protocol Constraints. The original DCCP-Request SHOULD also be reported in the Data Dropped option, either in a Normal Block (if the server accepted the data or 
there was no data) or in a Drop Code 0 Drop Block (if the server refused the data the first time as well).

The Data Dropped and Init Cookie options are particularly useful for DCCP-Response packets (Sections 11.7 and 8.1.4).

The server leaves the RESPOND state for OPEN when it receives a valid DCCP-Ack from the client, completing the three-way handshake. It MAY also leave the RESPOND state for CLOSED after a timeout of not less than 4MSL ( 8 minutes); when doing so, it SHOULD send a DCCP-Reset with Reset Code 2, "Aborted", to clean up state at the client.

\subsubsection{Init Cookie Option}

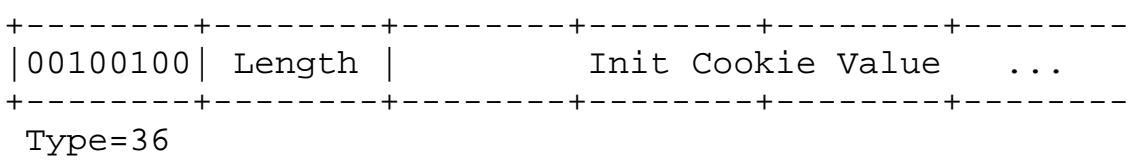

The Init Cookie option lets a DCCP server avoid having to hold any state until the three-way connection setup handshake has completed, in a similar fashion as for TCP SYN cookies [SYNCOOKIES]. The server wraps up the Service Code, server port, and any options it cares about from both the DCCP-Request and DCCP-Response in an opaque cookie. Typically the cookie will be encrypted using a secret known only to the server and will include a cryptographic checksum or magic value so that correct decryption can be verified. When the server receives the cookie back in the response, it can decrypt the cookie and instantiate all the state it avoided keeping. In the meantime, it need not move from the LISTEN state.

The Init Cookie option MUST NOT be sent on DCCP-Request or DCCP-Data packets. Any Init Cookie options received on DCCP-Request or DCCPData packets, or after the connection has been established (when the connection's state is $>=$ OPEN), MUST be ignored. The server MAY include Init Cookie options in its DCCP-Response. If so, then the client MUST echo the same Init Cookie options, in the same order, in each succeeding DCCP packet until one of those packets is acknowledged (showing that the three-way handshake has completed) or the connection is reset. As a result, the client MUST NOT use DCCPData packets until the three-way handshake completes or the connection is reset. The Init Cookie options on a client packet MUST equal those received on the $\mathrm{DCCP}-$ Request indicated by the client packet's Acknowledgement Number. The server SHould design its Init Cookie format so that Init Cookies can be checked for tampering; it SHOULD respond to a tampered Init Cookie option by resetting the connection with Reset Code 10, "Bad Init Cookie". 
Init Cookie's precise implementation need not be specified here; since Init cookies are opaque to the client, there are no interoperability concerns. An example cookie format might encrypt (using a secret key) the connection's initial sequence and acknowledgement numbers, ports, Service Code, any options included on the DCCP-Request packet and the corresponding DCCP-Response, a random salt, and a magic number. On receiving a reflected Init cookie, the server would decrypt the cookie, validate it by checking its magic number, sequence numbers, and ports, and, if valid, create a corresponding socket using the options.

Each individual Init Cookie option can hold at most 253 bytes of data, but a server can send multiple Init Cookie options to gain more space.

\subsubsection{Handshake Completion}

When the client receives a DCCP-Response from the server, it moves from the REQUEST state to PARTOPEN and completes the three-way handshake by sending a DCCP-Ack packet to the server. The client remains in PARTOPEN until it can be sure that the server has received some packet the client sent from PARTOPEN (either the initial DCCPAck or a later packet). Clients in the PARTOPEN state that want to send data MUST do so using DCCP-DataAck packets, not DCCP-Data packets. This is because DCCP-Data packets lack Acknowledgement Numbers, so the server can't tell from a DCCP-Data packet whether the client saw its DCCP-Response. Furthermore, if the DCCP-Response included an Init Cookie, that Init Cookie MUST be included on every packet sent in PARTOPEN.

The single DCCP-Ack sent when entering the PARTOPEN state might, of course, be dropped by the network. The client SHould ensure that some packet gets through eventually. The preferred mechanism would be a roughly 200-millisecond timer, set every time a packet is transmitted in PARTOPEN. If this timer goes off and the client is still in PARTOPEN, the client generates another DCCP-Ack and backs off the timer. If the client remains in PARTOPEN for more than 4MSL (8 minutes), it SHOULD reset the connection with Reset Code 2, "Aborted".

The client leaves the PARTOPEN state for OPEN when it receives a valid packet other than DCCP-Response, DCCP-Reset, or DCCP-Sync from the server.

\subsection{Data Transfer}

In the central data transfer phase of the connection, both server and client are in the OPEN state. 
DCCP A sends DCCP-Data and DCCP-DataAck packets to DCCP B due to application events on host $A$. These packets are congestioncontrolled by the CCID for the A-to-B half-connection. In contrast, DCCP-Ack packets sent by DCCP A are controlled by the CCID for the B-to-A half-connection. Generally, DCCP A will piggyback acknowledgement information on DCCP-Data packets when acceptable, creating DCCP-DataAck packets. DCCP-Ack packets are used when there is no data to send from DCCP A to DCCP B, or when the congestion state of the A-to-B CCID will not allow data to be sent.

DCCP-Sync and DCCP-SyncAck packets may also occur in the data transfer phase. Some cases causing DCCP-Sync generation are discussed in Section 7.5. One important distinction between DCCPSync packets and other packet types is that DCCP-Sync elicits an immediate acknowledgement. On receiving a valid DCCP-Sync packet, a DCCP endpoint MUST immediately generate and send a DCCP-SyncACk response (subject to any implementation rate limits); the Acknowledgement Number on that DCCP-SyncAck MUST equal the Sequence Number of the DCCP-Sync.

A particular DCCP implementation might decide to initiate feature negotiation only once the OPEN state was reached, in which case it might not allow data transfer until some time later. Data received during that time SHOULD be rejected and reported using a Data Dropped Drop Block with Drop Code 0, Protocol Constraints (see Section 11.7).

\subsection{Termination}

DCCP connection termination uses a handshake consisting of an optional DCCP-CloseReq packet, a DCCP-Close packet, and a DCCP-Reset packet. The server moves from the OPEN state, possibly through the CLOSEREQ state, to CLOSED; the client moves from OPEN through CLOSING to TIMEWAIT, and after 2MSL wait time ( 4 minutes) to CLOSED.

The sequence DCCP-CloseReq, DCCP-Close, DCCP-Reset is used when the server decides to close the connection but doesn't want to hold TIMEWAIT state:

\begin{tabular}{lllll}
$\begin{array}{c}\text { Client State } \\
\text { OPEN }\end{array}$ & & \multicolumn{2}{c}{$\begin{array}{c}\text { Server State } \\
\text { OPEN }\end{array}$} \\
1. & & CloseReq & $<--$ & CLOSEREQ \\
2. CLOSING & $-->$ & Close & $-->$ & \\
3. & & Reset & $<--$ & CLOSED (LISTEN) \\
4. TIMEWAIT & & & & \\
5. CLOSED & & &
\end{tabular}


A shorter sequence occurs when the client decides to close the connection.

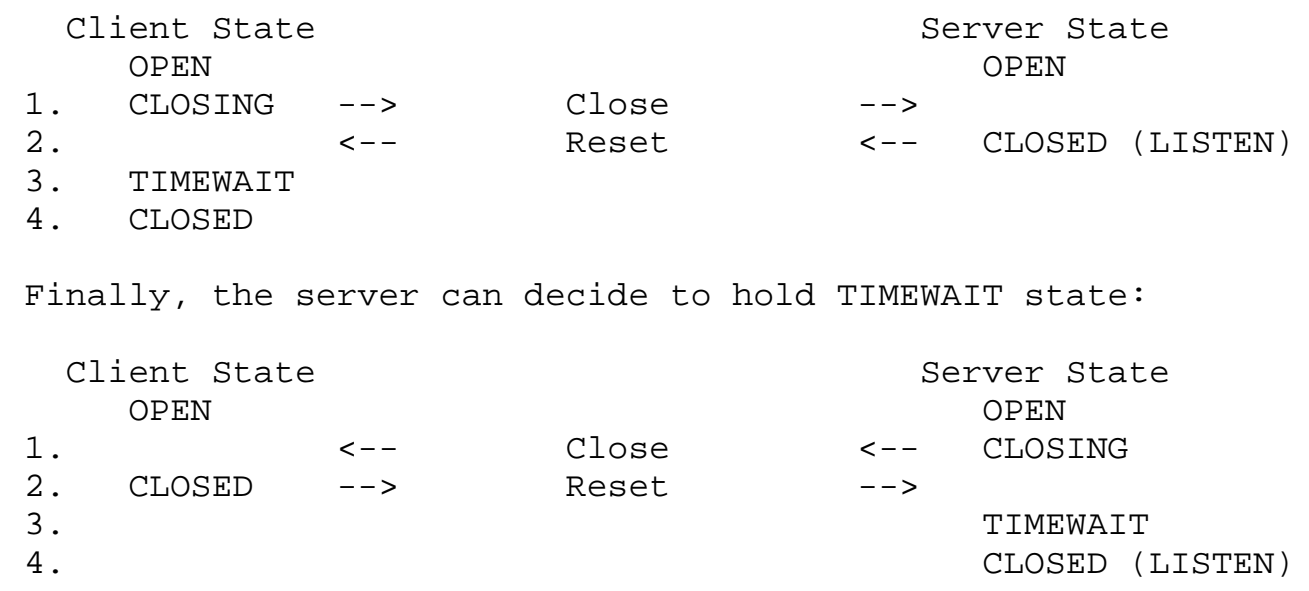

In all cases, the receiver of the DCCP-Reset packet holds TIMEWAIT state for the connection. As in TCP, TIMEWAIT state, where an endpoint quietly preserves a socket for 2MSL (4 minutes) after its connection has closed, ensures that no connection duplicating the current connection's source and destination addresses and ports can start up while old packets might remain in the network.

The termination handshake proceeds as follows. The receiver of a valid DCCP-CloseReq packet MUST respond with a DCCP-Close packet. The receiver of a valid DCCP-Close packet MUST respond with a DCCPReset packet with Reset Code 1, "Closed". The receiver of a valid DCCP-Reset packet -- which is also the sender of the DCCP-Close packet (and possibly the receiver of the DCCP-CloseReq packet) -will hold TIMEWAIT state for the connection.

A DCCP-Reset packet completes every DCCP connection, whether the termination is clean (due to application close; Reset Code 1, "Closed") or unclean. Unlike TCP, which has two distinct termination mechanisms (FIN and RST), DCCP ends all connections in a uniform manner. This is justified because some aspects of connection termination are the same independent of whether termination was clean. For instance, the endpoint that receives a valid DCCP-Reset SHOULD hold TIMEWAIT state for the connection. Processors that must distinguish between clean and unclean termination can examine the Reset Code. DCCP implementations generally transition to the CLOSED state after sending a DCCP-Reset packet.

Endpoints in the CLOSEREQ and CLOSING states MUST retransmit DCCPCloseReq and DCCP-Close packets, respectively, until leaving those 
states. The retransmission timer should initially be set to go off in two round-trip times and should back off to not less than once every 64 seconds if no relevant response is received.

Only the server can send a DCCP-CloseReq packet or enter the CLOSEREQ state. A server receiving a sequence-valid DCCP-CloseReq packet MUST respond with a DCCP-Sync packet and otherwise ignore the DCCPCloseReq.

DCCP-Data, DCCP-DataAck, and DCCP-Ack packets received in CLOSEREQ or CLOSING states MAY be either processed or ignored.

\subsubsection{Abnormal Termination}

DCCP endpoints generate DCCP-Reset packets to terminate connections abnormally; a DCCP-Reset packet may be generated from any state. Resets sent in the CLOSED, LISTEN, and TIMEWAIT states use Reset Code 3, "No Connection", unless otherwise specified. Resets sent in the REQUEST or RESPOND states use Reset Code 4, "Packet Error", unless otherwise specified.

DCCP endpoints in CLOSED, LISTEN, or TIMEWAIT state may need to generate a DCCP-Reset packet in response to a packet received from a peer. Since these states have no associated sequence number variables, the Sequence and Acknowledgement Numbers on the DCCP-Reset packet $R$ are taken from the received packet $P$, as follows.

1. If P.ackno exists, then set R.seqno := P.ackno + 1 . Otherwise, set $R$. seqno $:=0$.

2. Set R.ackno:= P. seqno.

3. If the packet used short sequence numbers ( $\mathrm{P} . \mathrm{X}==0$ ), then set the upper 24 bits of $R$.seqno and R. ackno to 0 .

8.4. DCCP State Diagram

The most common state transitions discussed above can be summarized in the following state diagram. The diagram is illustrative; the text in Section 8.5 and elsewhere should be considered definitive. For example, there are arcs (not shown) from every state except CLOSED to TIMEWAIT, contingent on the receipt of a valid DCCP-Reset. 


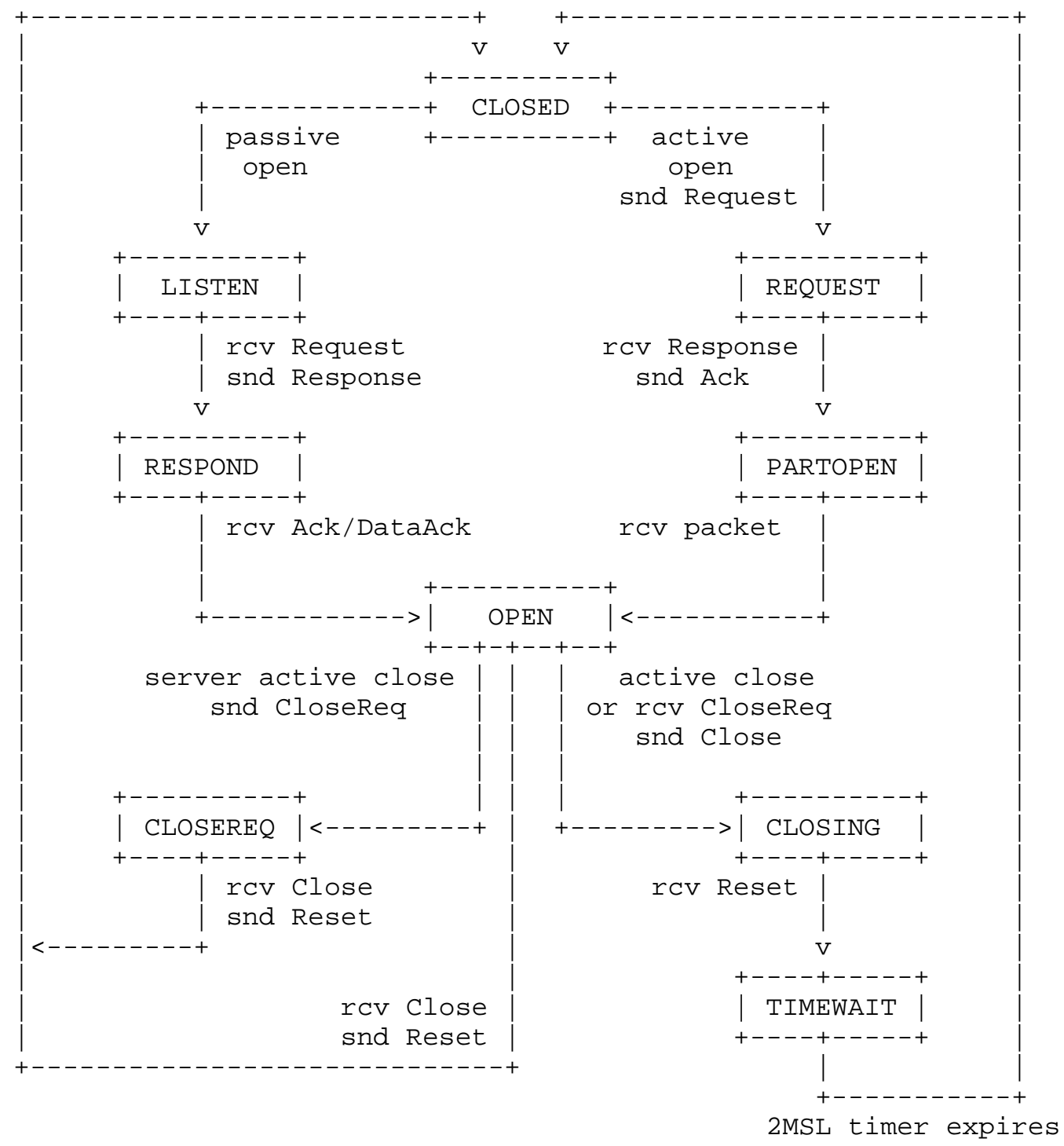

\subsection{Pseudocode}

This section presents an algorithm describing the processing steps a DCCP endpoint must go through when it receives a packet. A DCCP implementation need not implement the algorithm as it is described here, but any implementation MUST generate observable effects exactly as indicated by this pseudocode, except where allowed otherwise by another part of this document. 
The received packet is written as $\mathrm{P}$, the socket as $\mathrm{S}$. Socket variables are:

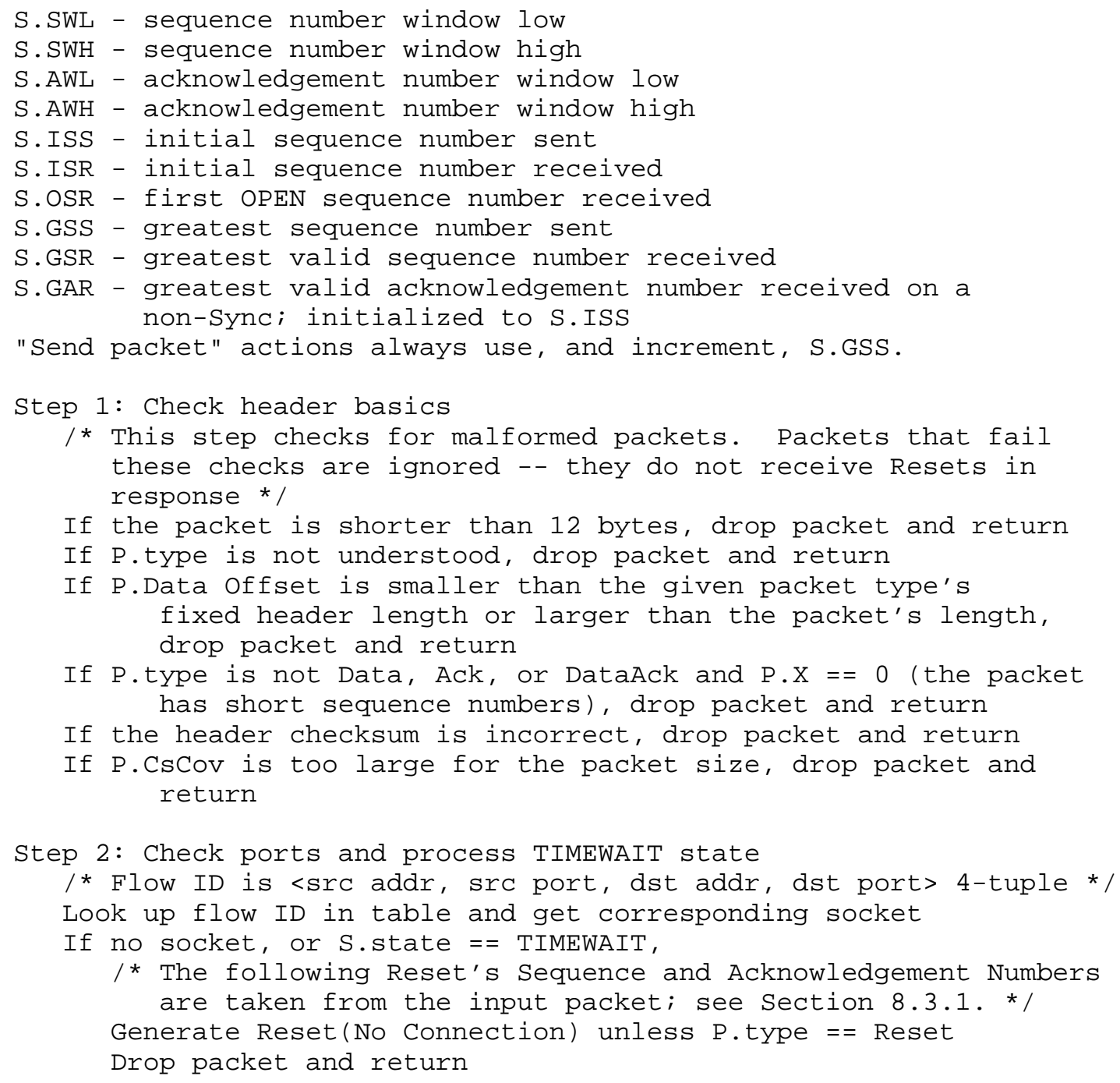




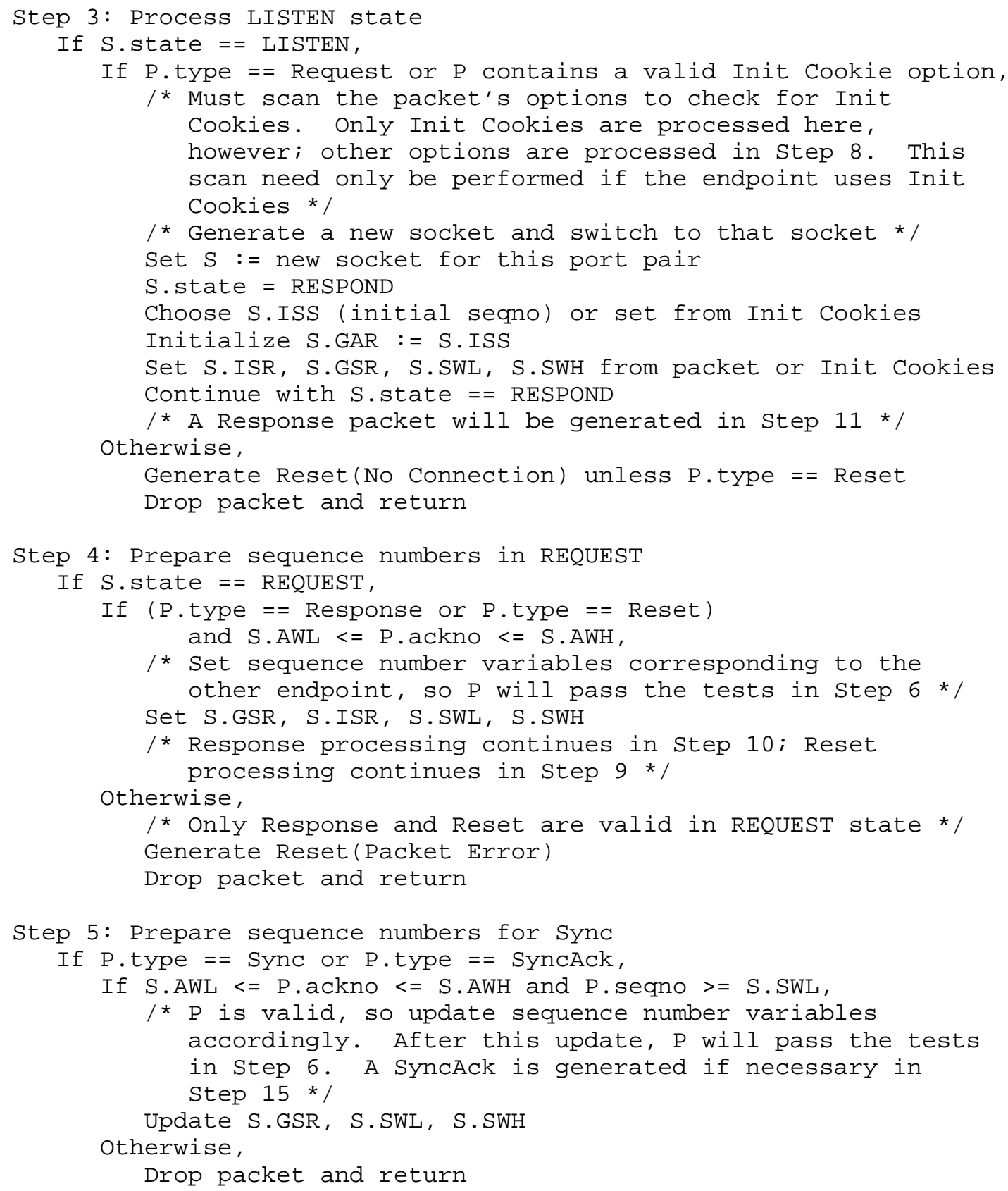




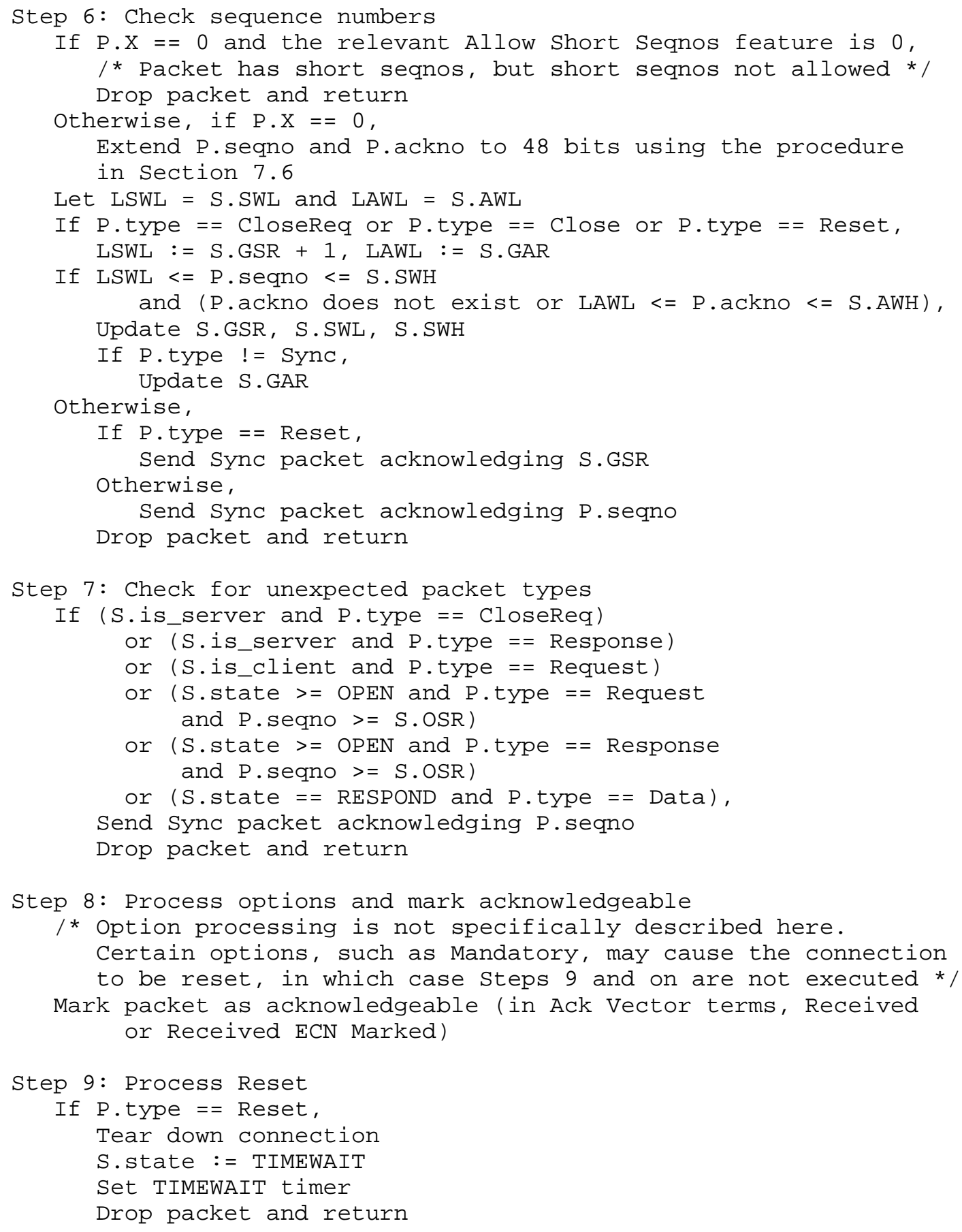




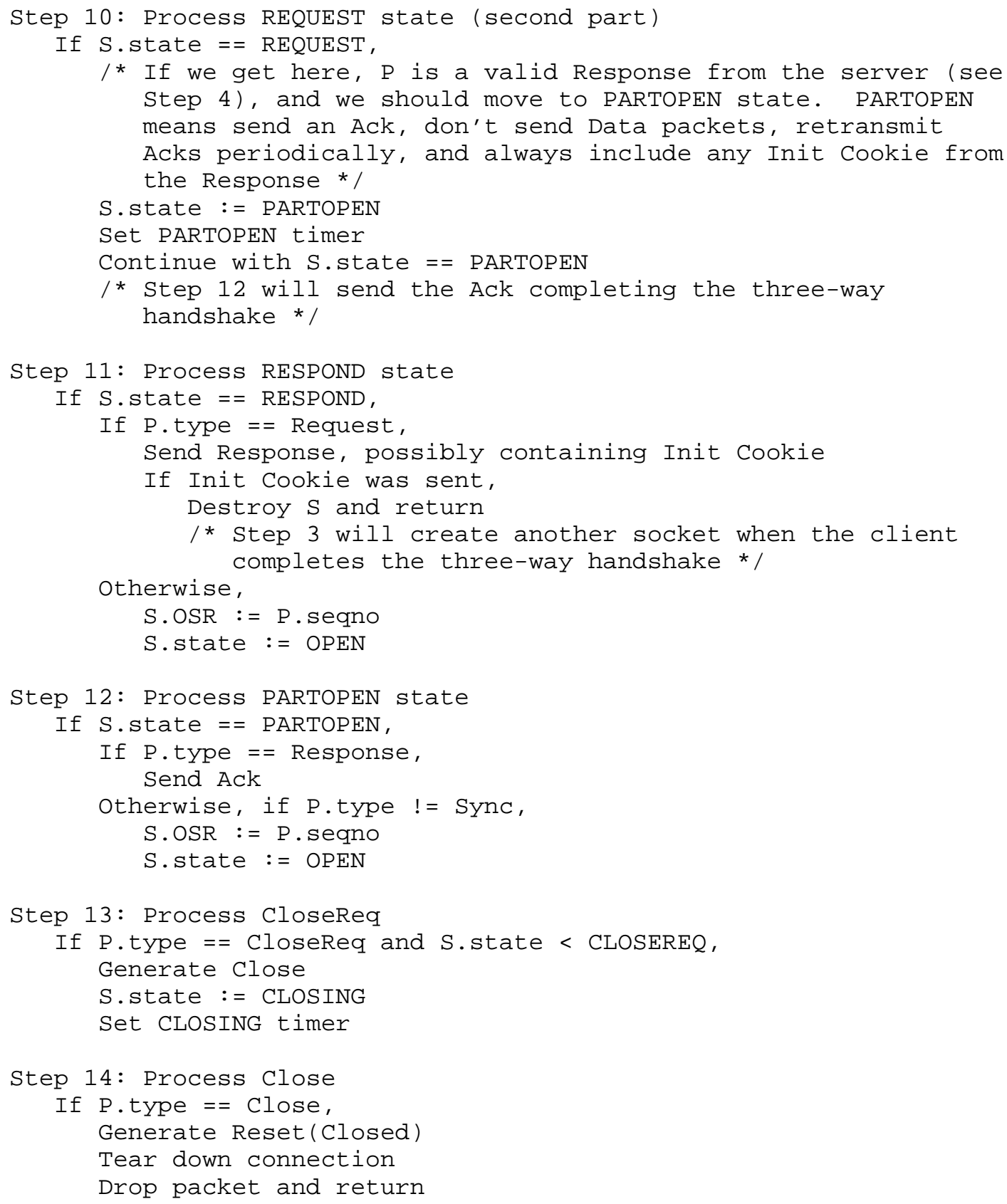




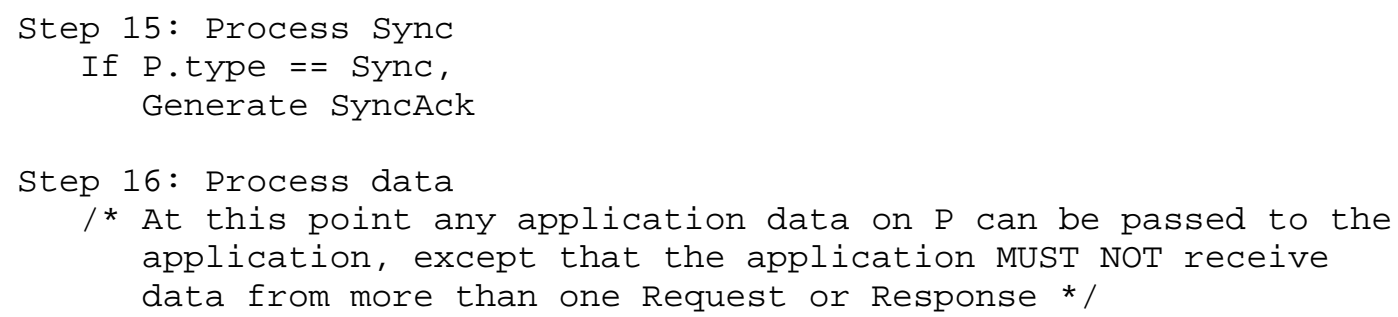

9. Checksums

\begin{abstract}
DCCP uses a header checksum to protect its header against corruption. Generally, this checksum also covers any application data. DCCP applications can, however, request that the header checksum cover only part of the application data, or perhaps no application data at all. Link layers may then reduce their protection on unprotected parts of DCCP packets. For some noisy links, and for applications that can tolerate corruption, this can greatly improve delivery rates and perceived performance.
\end{abstract}

Checksum coverage may eventually impact congestion control mechanisms as well. A packet with corrupt application data and complete checksum coverage is treated as lost. This incurs a heavy-duty loss response from the sender's congestion control mechanism, which can unfairly penalize connections on links with high background corruption. The combination of reduced checksum coverage and Data Checksum options may let endpoints report packets as corrupt rather than dropped, using Data Dropped options and Drop Code 3 (see section 11.7). This may eventually benefit applications. However, further research is required to determine an appropriate response to corruption, which can sometimes correlate with congestion. Corrupt packets currently incur a loss response.

The Data Checksum option, which contains a strong CRC, lets endpoints detect application data corruption. An API can then be used to avoid delivering corrupt data to the application, even if links deliver corrupt data to the endpoint due to reduced checksum coverage. However, the use of reduced checksum coverage for applications that demand correct data is currently considered experimental. This is because the combined loss-plus-corruption rate for packets with reduced checksum coverage may be significantly higher than that for packets with full checksum coverage, although the loss rate will generally be lower. Actual behavior will depend on link design; further research and experience is required.

Reduced checksum coverage introduces some security considerations; see Section 18.1. See Appendix B for further motivation and 
discussion. DCCP's implementation of reduced checksum coverage was inspired by UDP-Lite [RFC3828].

\subsection{Header Checksum Field}

DCCP uses the TCP/IP checksum algorithm. The Checksum field in the DCCP generic header (see Section 5.1) equals the 16-bit one's complement of the one's complement sum of all 16-bit words in the DCCP header, DCCP options, a pseudoheader taken from the networklayer header, and, depending on the value of the Checksum Coverage field, some or all of the application data. When calculating the checksum, the Checksum field itself is treated as 0 . If a packet contains an odd number of header and payload bytes to be checksummed, 8 zero bits are added on the right to form a 16-bit word for checksum purposes. The pad byte is not transmitted as part of the packet.

The pseudoheader is calculated as for TCP. For IPv4, it is 96 bits long and consists of the IPV4 source and destination addresses, the IP protocol number for DCCP (padded on the left with 8 zero bits), and the DCCP length as a 16-bit quantity (the length of the DCCP header with options, plus the length of any data); see [RFC793], Section 3.1. For IPv6, it is 320 bits long, and consists of the IPv6 source and destination addresses, the DCCP length as a 32-bit quantity, and the IP protocol number for DCCP (padded on the left with 24 zero bits); see [RFC2460], Section 8.1.

Packets with invalid header checksums MUST be ignored. In particular, their options MUST NOT be processed.

\subsection{Header Checksum Coverage Field}

The Checksum Coverage field in the DCCP generic header (see Section 5.1) specifies what parts of the packet are covered by the Checksum field, as follows:

$\mathrm{CsCov}=0$ The Checksum field covers the DCCP header, DCCP options, network-layer pseudoheader, and all application data in the packet, possibly padded on the right with zeros to an even number of bytes.

CsCov $=1-15$ The Checksum field covers the DCCP header, DCCP options, network-layer pseudoheader, and the initial $(\mathrm{CsCov}-1) * 4$ bytes of the packet's application data.

Thus, if CsCov is 1, none of the application data is protected by the header checksum. The value $\left(\mathrm{CsCov}^{-1}\right) * 4$ MUST be less than or equal to the length of the application data. Packets with invalid CsCov values MUST be ignored; in particular, their options MUST NOT be 
processed. The meanings of values other than 0 and 1 should be considered experimental.

Values other than 0 specify that corruption is acceptable in some or all of the DCCP packet's application data. In fact, DCCP cannot even detect corruption in areas not covered by the header checksum, unless the Data Checksum option is used. Applications should not make any assumptions about the correctness of received data not covered by the checksum and should, if necessary, introduce their own validity checks.

A DCCP application interface should let sending applications suggest a value for CsCov for sent packets, defaulting to 0 (full coverage). The Minimum Checksum Coverage feature, described below, lets an endpoint refuse delivery of application data on packets with partial checksum coverage; by default, only fully covered application data is accepted. Lower layers that support partial error detection MAY use the Checksum Coverage field as a hint of where errors do not need to be detected. Lower layers MUST use a strong error detection mechanism to detect at least errors that occur in the sensitive part of the packet, and to discard damaged packets. The sensitive part consists of the bytes between the first byte of the IP header and the last byte identified by Checksum Coverage.

For more details on application and lower-layer interface issues relating to partial checksumming, see [RFC3828].

\subsubsection{Minimum Checksum Coverage Feature}

The Minimum Checksum Coverage feature lets a DCCP endpoint determine whether its peer is willing to accept packets with reduced Checksum Coverage. For example, DCCP A sends a "Change R(Minimum Checksum Coverage, 1)" option to DCCP B to check whether B is willing to accept packets with Checksum Coverage set to 1 .

Minimum Checksum Coverage has feature number 8 and is serverpriority. It takes one-byte integer values between 0 and 15; values of 16 or more are reserved. Minimum Checksum Coverage/B reflects values of Checksum Coverage that DCCP B finds unacceptable. Say that the value of Minimum Checksum Coverage/B is MinCsCov. Then:

- If MinCsCov $=0$, then DCCP B only finds packets with CsCov $=0$ acceptable.

- If MinCsCov > 0, then DCCP B additionally finds packets with CsCov $>=$ MinCsCov acceptable. 
DCCP B MAY refuse to process application data from packets with unacceptable Checksum Coverage. Such packets SHOULD be reported using Data Dropped options (Section 11.7) with Drop Code 0, Protocol Constraints. New connections start with Minimum Checksum Coverage 0 for both endpoints.

\subsection{Data Checksum Option}

The Data Checksum option holds a 32-bit CRC-32c cyclic redundancycheck code of a DCCP packet's application data.

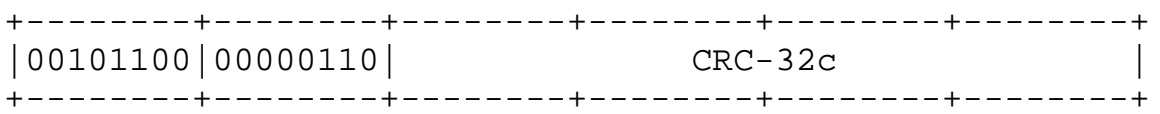

Type $=44$ Length $=6$

The sending DCCP computes the CRC of the bytes comprising the application data area and stores it in the option data. The CRC-32C algorithm used for Data Checksum is the same as that used for SCTP [RFC3309]; note that the CRC-32c of zero bytes of data equals zero. The DCCP header checksum will cover the Data Checksum option, so the data checksum must be computed before the header checksum.

A DCCP endpoint receiving a packet with a Data Checksum option either MUST or MAY check the Data Checksum; the choice depends on the value of the Check Data Checksum feature described below. If it checks the checksum, it computes the received application data's CRC-32c using the same algorithm as the sender and compares the result with the Data Checksum value. If the CRCs differ, the endpoint reacts in one of two ways:

- The receiving application may have requested delivery of knowncorrupt data via some optional API. In this case, the packet's data MUST be delivered to the application, with a note that it is known to be corrupt. Furthermore, the receiving endpoint MUST report the packet as delivered corrupt using a Data Dropped option (Drop Code 7, Delivered Corrupt).

o Otherwise, the receiving endpoint MUST drop the application data and report that data as dropped due to corruption using a Data Dropped option (Drop Code 3, Corrupt).

In either case, the packet is considered acknowledgeable (since its header was processed) and will therefore be acknowledged using the equivalent of Ack Vector's Received or Received ECN Marked states.

Although Data Checksum is intended for packets containing application data, it may be included on other packets, such as DCCP-ACk, DCCP- 
Sync, and DCCP-SyncAck. The receiver SHOULD calculate the application data area's CRC-32c on such packets, just as it does for DCCP-Data and similar packets. If the CRCs differ, the packets similarly MUST be reported using Data Dropped options (Drop Code 3), although their application data areas would not be delivered to the application in any case.

\subsubsection{Check Data Checksum Feature}

The Check Data Checksum feature lets a DCCP endpoint determine whether its peer will definitely check Data Checksum options. DCCP A sends a Mandatory "Change R(Check Data Checksum, 1)" option to DCCP B to require it to check Data Checksum options (the connection will be reset if it cannot).

Check Data Checksum has feature number 9 and is server-priority. It takes one-byte Boolean values. DCCP B MUST check any received Data Checksum options when Check Data Checksum/B is one, although it MAY check them even when Check Data Checksum/B is zero. Values of two or more are reserved. New connections start with Check Data Checksum 0 for both endpoints.

\subsubsection{Checksum Usage Notes}

Internet links must normally apply strong integrity checks to the packets they transmit [RFC3828, RFC3819]. This is the default case when the DCCP header's Checksum Coverage value equals zero (full coverage). However, the DCCP Checksum Coverage value might not be zero. By setting partial Checksum Coverage, the application indicates that it can tolerate corruption in the unprotected part of the application data. Recognizing this, link layers may reduce error detection and/or correction strength when transmitting this unprotected part. This, in turn, can significantly increase the likelihood of the endpoint's receiving corrupt data; Data Checksum lets the receiver detect that corruption with very high probability.

\section{Congestion Control}

Each congestion control mechanism supported by DCCP is assigned a congestion control identifier, or CCID: a number from 0 to 255. During connection setup, and optionally thereafter, the endpoints negotiate their congestion control mechanisms by negotiating the values for their Congestion Control ID features. Congestion Control ID has feature number 1. The CCID/A value equals the CCID in use for the A-to-B half-connection. DCCP B sends a "Change R(CCID, K)" option to ask DCCP A to use CCID $\mathrm{K}$ for its data packets. 
CCID is a server-priority feature, so CCID negotiation options can list multiple acceptable CCIDs, sorted in descending order of priority. For example, the option "Change R(CCID, 2 4)" asks the receiver to use CCID 2 for its packets, although CCIDs 3 and 4 are also acceptable. (This corresponds to the bytes "35, 6, 1, 2, 3, 4": Change $\mathrm{R}$ option (35), option length (6), feature ID (1), CCIDs (2, 3, 4).) Similarly, "Confirm L(CCID, 2, 2 3 4)" tells the receiver that the sender is using CCID 2 for its packets, but that CCIDs 3 and 4 might also be acceptable.

Currently allocated CCIDs are as follows:

$\begin{array}{clr}\text { CCID } & \text { Meaning } & \text { Reference } \\ ---- & ------ & ------- \\ 0-1 & \text { Reserved } & \\ 2 & \text { TCP-like Congestion Control } & \text { [RFC4341] } \\ 3 & \text { TCP-Friendly Rate Control } & \text { [RFC4342] } \\ 4-255 & \text { Reserved } & \\ & & \\ \text { Table 5: DCCP Congestion Control Identifiers }\end{array}$

New connections start with CCID 2 for both endpoints. If this is unacceptable for a DCCP endpoint, that endpoint MUST send Mandatory Change(CCID) options on its first packets.

All CCIDs standardized for use with DCCP will correspond to congestion control mechanisms previously standardized by the IETF. We expect that for quite some time, all such mechanisms will be TCP friendly, but TCP-friendliness is not an explicit DCCP requirement.

A DCCP implementation intended for general use, such as an implementation in a general-purpose operating system kernel, SHOULD implement at least CCID 2. The intent is to make CCID 2 broadly available for interoperability, although particular applications might disallow its use.

\subsection{TCP-like Congestion Control}

CCID 2, TCP-like Congestion Control, denotes Additive Increase, Multiplicative Decrease (AIMD) congestion control with behavior modelled directly on TCP, including congestion window, slow start, timeouts, and so forth [RFC2581]. CCID 2 achieves maximum bandwidth over the long term, consistent with the use of end-to-end congestion control, but halves its congestion window in response to each congestion event. This leads to the abrupt rate changes typical of TCP. Applications should use CCID 2 if they prefer maximum bandwidth utilization to steadiness of rate. This is often the case for applications that are not playing their data directly to the user. 
For example, a hypothetical application that transferred files over DCCP, using application-level retransmissions for lost packets, would prefer CCID 2 to CCID 3. On-line games may also prefer CCID 2.

CCID 2 is further described in [RFC4341].

10.2. TFRC Congestion Control

CCID 3 denotes TCP-Friendly Rate Control (TFRC), an equation-based rate-controlled congestion control mechanism. TFRC is designed to be reasonably fair when competing for bandwidth with TCP-like flows, where a flow is "reasonably fair" if its sending rate is generally within a factor of two of the sending rate of a TCP flow under the same conditions. However, TFRC has a much lower variation of throughput over time compared with TCP, which makes CCID 3 more suitable than CCID 2 for applications such as streaming media where a relatively smooth sending rate is important.

CCID 3 is further described in [RFC4342]. The TFRC congestion control algorithms were initially described in [RFC3448].

10.3. CCID-Specific Options, Features, and Reset Codes

Half of the option types, feature numbers, and Reset Codes are reserved for CCID-specific use. CCIDs may often need new options, for communicating acknowledgement or rate information, for example; reserved option spaces let CCIDs create options at will without polluting the global option space. Option 128 might have different meanings on a half-connection using CCID 4 and a half-connection using CCID 8. CCID-specific options and features will never conflict with global options and features introduced by later versions of this specification.

Any packet may contain information meant for either half-connection, so CCID-specific option types, feature numbers, and Reset Codes explicitly signal the half-connection to which they apply.

- Option numbers 128 through 191 are for options sent from the HC-Sender to the HC-Receiver; option numbers 192 through 255 are for options sent from the HC-Receiver to the HC-Sender.

- Reset Codes 128 through 191 indicate that the HC-Sender reset the connection (most likely because of some problem with acknowledgements sent by the HC-Receiver). Reset Codes 192 through 255 indicate that the HC-Receiver reset the connection (most likely because of some problem with data packets sent by the HC-Sender). 
- Finally, feature numbers 128 through 191 are used for features located at the HC-Sender; feature numbers 192 through 255 are for features located at the HC-Receiver. Since Change L and Confirm L options for a feature are sent by the feature location, we know that any Change L(128) option was sent by the HC-Sender, while any Change L(192) option was sent by the HC-Receiver. Similarly, Change $\mathrm{R}(128)$ options are sent by the HC-Receiver, while Change $\mathrm{R}(192)$ options are sent by the HC-Sender.

For example, consider a DCCP connection where the A-to-B halfconnection uses CCID 4 and the B-to-A half-connection uses CCID 5. Here is how a sampling of CCID-specific options are assigned to half-connections.

\begin{tabular}{|c|c|c|c|c|c|}
\hline \multirow{2}{*}{\multicolumn{2}{|c|}{ Packet }} & \multicolumn{3}{|r|}{ Relevant } & \multirow{2}{*}{$\begin{array}{l}\text { Relevant } \\
\text { CCID }\end{array}$} \\
\hline & & Option & & Half-conn. & \\
\hline & ----- & ------ & & ---------- & ---- \\
\hline & $>\mathrm{B}$ & 128 & & $A-t o-B$ & 4 \\
\hline & $>\mathrm{B}$ & 192 & & $B-t o-A$ & 5 \\
\hline A & $>\mathrm{B}$ & Change L (128, & $\ldots)$ & $A-$ to $-B$ & 4 \\
\hline A & $>\mathrm{B}$ & Change R(192, & $\ldots)$ & $A-t o-B$ & 4 \\
\hline & $>\mathrm{B}$ & Confirm L(128, & $\ldots)$ & $A-t o-B$ & 4 \\
\hline & $>\mathrm{B}$ & Confirm R(192, & $\ldots)$ & $A-t o-B$ & 4 \\
\hline & $>\mathrm{B}$ & Change R(128, & $\ldots)$ & $B-t o-A$ & 5 \\
\hline A & $>\mathrm{B}$ & Change L (192, & $\ldots)$ & $B-t o-A$ & 5 \\
\hline & $>\mathrm{B}$ & Confirm R(128, & $\ldots)$ & $B-$ to-A & 5 \\
\hline & $>\mathrm{B}$ & Confirm L(192, & $\ldots)$ & $B-t o-A$ & 5 \\
\hline & $>\mathrm{A}$ & 128 & & $B-$ to-A & 5 \\
\hline & $>A$ & 192 & & $A-t o-B$ & 4 \\
\hline B & $>A$ & Change L (128, & $\ldots)$ & $B-t o-A$ & 5 \\
\hline & $>A$ & Change R(192, & $\ldots)$ & $B-t o-A$ & 5 \\
\hline & $>\mathrm{A}$ & Confirm L (128, & $\ldots)$ & $B-t o-A$ & 5 \\
\hline & $>\mathrm{A}$ & Confirm R(192, & $\ldots)$ & $B-$ to-A & 5 \\
\hline B & $>\mathrm{A}$ & Change R(128, & $\ldots)$ & $A-t o-B$ & 4 \\
\hline B & $>\mathrm{A}$ & Change L (192, & $\ldots)$ & $A-t o-B$ & 4 \\
\hline & $>\mathrm{A}$ & Confirm R(128, & $\ldots)$ & $A-t o-B$ & 4 \\
\hline & $>\mathrm{A}$ & Confirm L (192, & $\ldots)$ & $A-t o-B$ & 4 \\
\hline
\end{tabular}

Using CCID-specific options and feature options during a negotiation for the corresponding CCID feature is NOT RECOMMENDED, since it is difficult to predict which CCID will be in force when the option is processed. For example, if a DCCP-Request contains the option sequence "Change L(CCID, 3), 128", the CCID-specific option "128" may be processed either by CCID 3 (if the server supports CCID 3) or by the default CCID 2 (if it does not). However, it is safe to include CCID-specific options following certain Mandatory Change(CCID) 
options. For example, if a DCCP-Request contains the option sequence "Mandatory, Change L(CCID, 3), 128", then either the "128" option will be processed by CCID 3 or the connection will be reset.

Servers that do not implement the default CCID 2 might nevertheless receive CCID 2-specific options on a DCCP-Request packet. (Such a server MUST send Mandatory Change(CCID) options on its DCCP-Response, so CCID-specific options on any other packet won't refer to CCID 2.) The server MUST treat such options as non-understood. Thus, it will reset the connection on encountering a Mandatory CCID-specific option or feature negotiation request, send an empty Confirm for a nonMandatory Change option for a CCID-specific feature, and ignore other CCID-specific options.

10.4. CCID Profile Requirements

Each CCID Profile document MUST address at least the following requirements:

- The profile MUST include the name and number of the CCID being described.

- The profile MUST describe the conditions in which it is likely to be useful. Often the best way to do this is by comparison to existing CCIDs.

- The profile MUST list and describe any CCID-specific options, features, and Reset Codes and SHOULD list those general options and features described in this document that are especially relevant to the CCID.

- Any newly defined acknowledgement mechanism MUST include a way to transmit ECN Nonce Echoes back to the sender.

- The profile MUST describe the format of data packets, including any options that should be included and the setting of the CCval header field.

- The profile MUST describe the format of acknowledgement packets, including any options that should be included.

- The profile MUST define how data packets are congestion controlled. This includes responses to congestion events, to idle and application-limited periods, and to the DCCP Data Dropped and Slow Receiver options. CCIDs that implement per-packet congestion control sHould discuss how packet size is factored in to congestion control decisions. 
- The profile MUST specify when acknowledgement packets are generated and how they are congestion controlled.

- The profile MUST define when a sender using the CCID is considered quiescent.

- The profile MUST say whether its CCID's acknowledgements ever need to be acknowledged and, if so, how often.

\subsection{Congestion state}

Most congestion control algorithms depend on past history to determine the current allowed sending rate. In CCID 2, this congestion state includes a congestion window and a measurement of the number of packets outstanding in the network; in CCID 3, it includes the lengths of recent loss intervals. Both CCIDs use an estimate of the round-trip time. Congestion state depends on the network path and is invalidated by path changes. Therefore, DCCP senders and receivers SHOULD reset their congestion state -essentially restarting congestion control from "slow start" or equivalent -- on significant changes in the end-to-end path. For example, an endpoint that sends or receives a Mobile IPv6 Binding Update message [RFC3775] SHOULD reset its congestion state for any corresponding DCCP connections.

A DCCP implementation MAY also reset its congestion state when a CCID changes (that is, when a negotiation for the CCID feature completes successfully and the new feature value differs from the old value). Thus, a connection in a heavily congested environment might evade end-to-end congestion control by frequently renegotiating a CCID, just as it could evade end-to-end congestion control by opening new connections for the same session. This behavior is prohibited. To prevent it, DCCP implementations MAY limit the rate at which CCID can be changed -- for instance, by refusing to change a CCID feature value more than once per minute.

\section{Acknowledgements}

Congestion control requires that receivers transmit information about packet losses and ECN marks to senders. DCCP receivers MUST report all congestion they see, as defined by the relevant CCID profile. Each CCID says when acknowledgements should be sent, what options they must use, and so on. DCCP acknowledgements are congestion controlled, although it is not required that the acknowledgement stream be more than very roughly TCP friendly; each CCID defines how acknowledgements are congestion controlled. 
Most acknowledgements use DCCP options. For example, on a halfconnection with CCID 2 (TCP-like), the receiver reports acknowledgement information using the Ack Vector option. This section describes common acknowledgement options and shows how acks using those options will commonly work. Full descriptions of the ack mechanisms used for each CCID are laid out in the CCID profile specifications.

Acknowledgement options, such as Ack Vector, depend on the DCCP Acknowledgement Number and are thus only allowed on packet types that carry that number. Acknowledgement options received on other packet types, namely DCCP-Request and DCCP-Data, MUST be ignored. Detailed acknowledgement options are not necessarily required on every packet that carries an Acknowledgement Number, however.

\subsection{Acks of Acks and Unidirectional Connections}

DCCP was designed to work well for both bidirectional and unidirectional flows of data, and for connections that transition between these states. However, acknowledgements required for a unidirectional connection are very different from those required for a bidirectional connection. In particular, unidirectional connections need to worry about acks of acks.

The ack-of-acks problem arises because some acknowledgement mechanisms are reliable. For example, an HC-Receiver using CCID 2, TCP-like Congestion Control, sends Ack Vectors containing completely reliable acknowledgement information. The HC-Sender should occasionally inform the HC-Receiver that it has received an ack. If it did not, the HC-Receiver might resend complete Ack Vector information, going back to the start of the connection, with every DCCP-Ack packet! However, note that acks-of-acks need not be reliable themselves: when an ack-of-acks is lost, the HC-Receiver will simply maintain, and periodically retransmit, old acknowledgement-related state for a little longer. Therefore, there is no need for acks-of-acks-of-acks.

When communication is bidirectional, any required acks-of-acks are automatically contained in normal acknowledgements for data packets. On a unidirectional connection, however, the receiver DCCP sends no data, so the sender would not normally send acknowledgements. Therefore, the CCID in force on that half-connection must explicitly say whether, when, and how the HC-Sender should generate acks-ofacks.

For example, consider a bidirectional connection where both halfconnections use the same CCID (either 2 or 3), and where DCCP B goes "quiescent". This means that the connection becomes unidirectional: 
DCCP B stops sending data and sends only DCCP-Ack packets to DCCP A. In CCID 2, TCP-like Congestion Control, DCCP B uses Ack Vector to reliably communicate which packets it has received. As described above, DCCP A must occasionally acknowledge a pure acknowledgement from DCCP B so that B can free old Ack Vector state. For instance, A might send a DCCP-DataAck packet instead of DCCP-Data every now and then. In CCID 3, however, acknowledgement state is generally bounded, so A does not need to acknowledge $B^{\prime}$ s acknowledgements.

When communication is unidirectional, a single CCID -- in the example, the A-to-B CCID -- controls both DCCPs' acknowledgements, in terms of their content, their frequency, and so forth. For bidirectional connections, the A-to-B CCID governs DCCP B's acknowledgements (including its acks of DCCP $A^{\prime} s$ acks) and the $B-$ to-A CCID governs DCCP A's acknowledgements.

DCCP A switches its ack pattern from bidirectional to unidirectional when it notices that DCCP B has gone quiescent. It switches from unidirectional to bidirectional when it must acknowledge even a single DCCP-Data or DCCP-DataAck packet from DCCP B.

Each CCID defines how to detect quiescence on that CCID, and how that CCID handles acks-of-acks on unidirectional connections. The B-to-A CCID defines when DCCP B has gone quiescent. Usually, this happens when a period has passed without B sending any data packets; in CCID 2, for example, this period is the maximum of 0.2 seconds and two round-trip times. The A-to-B CCID defines how DCCP A handles acks-of-acks once DCCP B has gone quiescent.

\subsection{Ack Piggybacking}

Acknowledgements of A-to-B data MAY be piggybacked on data sent by DCCP B, as long as that does not delay the acknowledgement longer than the A-to-B CCID would find acceptable. However, data acknowledgements often require more than 4 bytes to express. A large set of acknowledgements prepended to a large data packet might exceed the allowed maximum packet size. In this case, DCCP B SHOULD send separate DCCP-Data and DCCP-ACk packets, or wait, but not too long, for a smaller datagram.

Piggybacking is particularly common at DCCP A when the B-to-A half-connection is quiescent -- that is, when DCCP A is just acknowledging DCCP $B^{\prime} s$ acknowledgements. There are three reasons to acknowledge DCCP $B^{\prime}$ s acknowledgements: to allow DCCP B to free up information about previously acknowledged data packets from A; to shrink the size of future acknowledgements; and to manipulate the rate at which future acknowledgements are sent. Since these are 
secondary concerns, DCCP A can generally afford to wait indefinitely for a data packet to piggyback its acknowledgement onto; if DCCP B wants to elicit an acknowledgement, it can send a DCCP-Sync.

Any restrictions on ack piggybacking are described in the relevant CCID's profile.

\subsection{Ack Ratio Feature}

The Ack Ratio feature lets HC-Senders influence the rate at which HC-Receivers generate DCCP-Ack packets, thus controlling reverse-path congestion. This differs from TCP, which presently has no congestion control for pure acknowledgement traffic. Ack Ratio reverse-path congestion control does not try to be TCP friendly. It just tries to avoid congestion collapse, and to be somewhat better than TCP in the presence of a high packet loss or mark rate on the reverse path.

Ack Ratio applies to CCIDs whose HC-Receivers clock acknowledgements off the receipt of data packets. The value of Ack Ratio/A equals the rough ratio of data packets sent by DCCP A to DCCP-Ack packets sent by DCCP B. Higher Ack Ratios correspond to lower DCCP-Ack rates; the sender raises Ack Ratio when the reverse path is congested and lowers Ack Ratio when it is not. Each CCID profile defines how it controls congestion on the acknowledgement path, and, particularly, whether Ack Ratio is used. CCID 2, for example, uses Ack Ratio for acknowledgement congestion control, but CCID 3 does not. However, each Ack Ratio feature has a value whether or not that value is used by the relevant CCID.

Ack Ratio has feature number 5 and is non-negotiable. It takes twobyte integer values. An Ack Ratio/A value of four means that DCCP B will send at least one acknowledgement packet for every four data packets sent by DCCP A. DCCP A sends a "Change L (Ack Ratio)" option to notify DCCP B of its ack ratio. An Ack Ratio value of zero indicates that the relevant half-connection does not use an Ack Ratio to control its acknowledgement rate. New connections start with Ack Ratio 2 for both endpoints; this Ack Ratio results in acknowledgement behavior analogous to TCP's delayed acks.

Ack Ratio should be treated as a guideline rather than a strict requirement. We intend Ack Ratio-controlled acknowledgement behavior to resemble $\mathrm{TCP}^{\prime} \mathrm{s}$ acknowledgement behavior when there is no reversepath congestion, and to be somewhat more conservative when there is reverse-path congestion. Following this intent is more important than implementing Ack Ratio precisely. In particular: 
- Receivers MAY piggyback acknowledgement information on data packets, creating DCCP-DataAck packets. The Ack Ratio does not apply to piggybacked acknowledgements. However, if the data packets are too big to carry acknowledgement information, or if the data sending rate is lower than Ack Ratio would suggest, then DCCP B SHOULD send enough pure DCCP-Ack packets to maintain the rate of one acknowledgement per Ack Ratio received data packets.

- Receivers MAY rate-pace their acknowledgements rather than send acknowledgements immediately upon the receipt of data packets. Receivers that rate-pace acknowledgements SHOULD pick a rate that approximates the effect of Ack Ratio and SHOULD include Elapsed Time options (Section 13.2) to help the sender calculate roundtrip times.

- Receivers SHOULD implement delayed acknowledgement timers like $\mathrm{TCP}^{\prime} \mathrm{s}$, whereby any packet's acknowledgement is delayed by at most $T$ seconds. This delay lets the receiver collect additional packets to acknowledge and thus reduce the per-packet overhead of acknowledgements; but if $\mathrm{T}$ seconds have passed by and the ack is still around, it is sent out right away. The default value of $T$ should be 0.2 seconds, as is common in TCP implementations. This may lead to sending more acknowledgement packets than Ack Ratio would suggest.

- Receivers SHOULD send acknowledgements immediately on receiving packets marked ECN Congestion Experienced or packets whose outof-order sequence numbers potentially indicate loss. However, there is no need to send such immediate acknowledgements for marked packets more than once per round-trip time.

- Receivers MAY ignore Ack Ratio if they perform their own congestion control on acknowledgements. For example, a receiver that knows the loss and mark rate for its DCCP-Ack packets might maintain a TCP-friendly acknowledgement rate on its own. Such a receiver MUST either ensure that it always obtains sufficient acknowledgement loss and mark information or fall back to Ack Ratio when sufficient information is not available, as might happen during periods when the receiver is quiescent.

\subsection{Ack Vector Options}

The Ack Vector gives a run-length encoded history of data packets received at the client. Each byte of the vector gives the state of that data packet in the loss history, and the number of preceding packets with the same state. The option's data looks like this: 


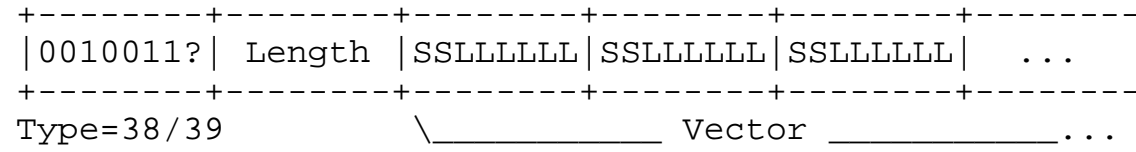

The two Ack Vector options (option types 38 and 39) differ only in the values they imply for ECN Nonce Echo. Section 12.2 describes this further.

The vector itself consists of a series of bytes, each of whose encoding is:

$\begin{array}{llllllll}0 & 1 & 2 & 3 & 4 & 5 & 6 & 7\end{array}$

$+-+-+-+-+-+-+-+-+$

Sta| Run Length |

$+-+-+-+-+-+-+-+-+$

Sta[te] occupies the most significant two bits of each byte and can have one of four values, as follows:

$\begin{array}{cl}\text { State } & \text { Meaning } \\ ---- & ------ \\ 0 & \text { Received } \\ 1 & \text { Received ECN Marked } \\ 2 & \text { Reserved } \\ 3 & \text { Not Yet Received } \\ \text { Table 6: } & \text { DCCP Ack Vector States }\end{array}$

The term "ECN marked" refers to packets with ECN code point 11, CE (Congestion Experienced); packets received with this ECN code point MUST be reported using State 1, Received ECN Marked. Packets received with ECN code points 00, 01, or 10 (Non-ECT, ECT(0), or ECT(1), respectively) MUST be reported using state 0, Received.

Run Length, the least significant six bits of each byte, specifies how many consecutive packets have the given state. Run Length zero says the corresponding State applies to one packet only; Run Length 63 says it applies to 64 consecutive packets. Run lengths of 65 or more must be encoded in multiple bytes.

The first byte in the first Ack Vector option refers to the packet indicated in the Acknowledgement Number; subsequent bytes refer to older packets. Ack Vector MUST NOT be sent on DCCP-Data and DCCPRequest packets, which lack an Acknowledgement Number, and any Ack Vector options encountered on such packets MUST be ignored. 
An Ack Vector containing the decimal values 0,192,3,64,5 and for which the Acknowledgement Number is decimal 100 indicates that:

Packet 100 was received (Acknowledgement Number 100, state 0, Run Length 0$)$;

Packet 99 was lost (State 3, Run Length 0);

Packets 98, 97, 96 and 95 were received (State 0, Run Length 3);

Packet 94 was ECN marked (State 1, Run Length 0); and

Packets 93, 92, 91, 90, 89, and 88 were received (State 0, Run Length 5).

A single Ack Vector option can acknowledge up to 16192 data packets. Should more packets need to be acknowledged than can fit in 253 bytes of Ack Vector, then multiple Ack Vector options can be sent; the second Ack Vector begins where the first left off, and so forth.

Ack Vector states are subject to two general constraints. (These principles SHOULD also be followed for other acknowledgement mechanisms; referring to Ack Vector states simplifies their explanation.)

1. Packets reported as State 0 or State 1 MUST be acknowledgeable: their options have been processed by the receiving DCCP stack. Any data on the packet need not have been delivered to the receiving application; in fact, the data may have been dropped.

2. Packets reported as state 3 MUST NOT be acknowledgeable. Feature negotiations and options on such packets MUST NOT have been processed, and the Acknowledgement Number MUST NOT correspond to such a packet.

Packets dropped in the application's receive buffer MUST be reported as Received or Received ECN Marked (States 0 and 1), depending on their ECN state; such packets' ECN Nonces MUST be included in the Nonce Echo. The Data Dropped option informs the sender that some packets reported as received actually had their application data dropped.

One or more Ack Vector options that, together, report the status of a packet with a sequence number less than ISN, the initial sequence number, SHOULD be considered invalid. The receiving DCCP SHOULD either ignore the options or reset the connection with Reset Code 5, "Option Error". No Ack Vector option can refer to a packet that has not yet been sent, as the Acknowledgement Number checks in section 
7.5.3 ensure, but because of attack, implementation bug, or misbehavior, an Ack Vector option can claim that a packet was received before it is actually delivered. Section 12.2 describes how this is detected and how senders should react. Packets that haven't been included in any Ack Vector option SHOULD be treated as "not yet received" (State 3) by the sender.

Appendix A provides a non-normative description of the details of DCCP acknowledgement handling in the context of an abstract Ack Vector implementation.

\subsubsection{Ack Vector Consistency}

A DCCP sender will commonly receive multiple acknowledgements for some of its data packets. For instance, an HC-Sender might receive two DCCP-Acks with Ack Vectors, both of which contained information about sequence number 24. (Information about a sequence number is generally repeated in every ack until the HC-Sender acknowledges an ack. In this case, perhaps the HC-Receiver is sending acks faster than the HC-Sender is acknowledging them.) In a perfect world, the two Ack Vectors would always be consistent. However, there are many reasons why they might not be. For example:

- The HC-Receiver received packet 24 between sending its acks, so the first ack said 24 was not received (State 3) and the second said it was received or ECN marked (State 0 or 1 ).

- The HC-Receiver received packet 24 between sending its acks, and the network reordered the acks. In this case, the packet will appear to transition from state 0 or 1 to state 3.

- The network duplicated packet 24, and one of the duplicates was ECN marked. This might show up as a transition between states 0 and 1 .

To cope with these situations, HC-Sender DCCP implementations SHOULD combine multiple received Ack Vector states according to this table:

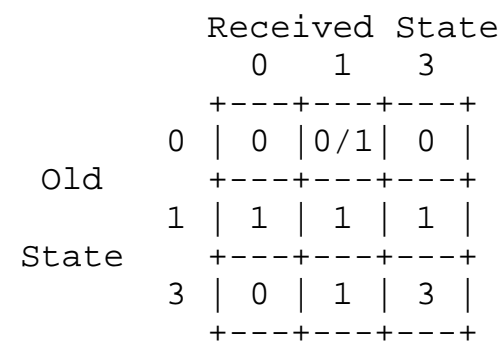


To read the table, choose the row corresponding to the packet's old state and the column corresponding to the packet's state in the newly received Ack Vector; then read the packet's new state off the table. For an old state of 0 (received non-marked) and received state of 1 (received ECN marked), the packet's new state may be set to either 0 or 1. The HC-Sender implementation will be indifferent to ack reordering if it chooses new state 1 for that cell.

The HC-Receiver should collect information about received packets according to the following table:

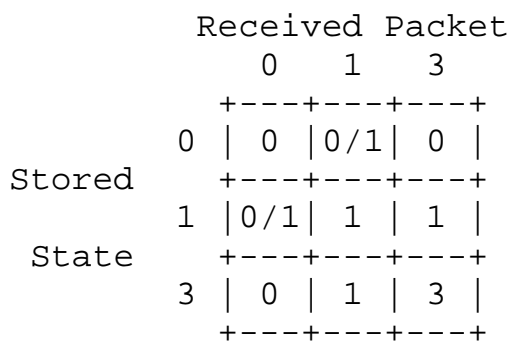

This table equals the sender's table except that, when the stored state is 1 and the received state is 0 , the receiver is allowed to switch its stored state to 0 .

An HC-Sender MAY choose to throw away old information gleaned from the HC-Receiver's Ack Vectors, in which case it MUST ignore newly received acknowledgements from the HC-Receiver for those old packets. It is often kinder to save recent Ack Vector information for a while so that the $\mathrm{HC}$-Sender can undo its reaction to presumed congestion when a "lost" packet unexpectedly shows up (the transition from state 3 to state 0).

\subsubsection{Ack Vector Coverage}

We can divide the packets that have been sent from an HC-Sender to an HC-Receiver into four roughly contiguous groups. From oldest to youngest, these are:

1. Packets already acknowledged by the HC-Receiver, where the HC-Receiver knows that the HC-Sender has definitely received the acknowledgements;

2. Packets already acknowledged by the HC-Receiver, where the HC-Receiver cannot be sure that the HC-Sender has received the acknowledgements;

3. Packets not yet acknowledged by the HC-Receiver; and 
4. Packets not yet received by the HC-Receiver.

The union of groups 2 and 3 is called the Acknowledgement Window. Generally, every Ack Vector generated by the HC-Receiver will cover the whole Acknowledgement Window: Ack Vector acknowledgements are cumulative. (This simplifies Ack Vector maintenance at the HC-Receiver; see Appendix A, below.) As packets are received, this window both grows on the right and shrinks on the left. It grows because there are more packets, and shrinks because the HC-Sender's Acknowledgement Numbers will acknowledge previous acknowledgements, moving packets from group 2 into group 1 .

\subsection{Send Ack Vector Feature}

The Send Ack Vector feature lets DCCPs negotiate whether they should use Ack Vector options to report congestion. Ack Vector provides detailed loss information and lets senders report back to their applications whether particular packets were dropped. Send Ack Vector is mandatory for some CCIDs and optional for others.

Send Ack Vector has feature number 6 and is server-priority. It takes one-byte Boolean values. DCCP A MUST send Ack Vector options on its acknowledgements when Send Ack Vector/A has value one, although it MAY send Ack Vector options even when send Ack Vector/A is zero. Values of two or more are reserved. New connections start with Send Ack Vector 0 for both endpoints. DCCP B sends a "Change R(Send Ack Vector, 1)" option to DCCP A to ask A to send Ack Vector options as part of its acknowledgement traffic.

\subsection{Slow Receiver Option}

An HC-Receiver sends the Slow Receiver option to its sender to indicate that it is having trouble keeping up with the sender's data. The HC-Sender SHOULD NOT increase its sending rate for approximately one round-trip time after seeing a packet with a slow Receiver option. After one round-trip time, the effect of Slow Receiver disappears, allowing the HC-Sender to increase its rate. Therefore, the HC-Receiver SHOULD continue to send Slow Receiver options if it needs to prevent the HC-Sender from going faster in the long term. The Slow Receiver option does not indicate congestion, and the HCSender need not reduce its sending rate. (If necessary, the receiver can force the sender to slow down by dropping packets, with or without Data Dropped, or by reporting false ECN marks.) APIs should let receiver applications set Slow Receiver and sending applications determine whether their receivers are slow. 
Slow Receiver is a one-byte option.

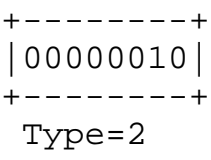

Slow Receiver does not specify why the receiver is having trouble keeping up with the sender. Possible reasons include lack of buffer space, CPU overload, and application quotas. A sending application might react to slow Receiver by reducing its application-level sending rate, for example.

The sending application should not react to slow Receiver by sending more data, however. Although the optimal response to a CPU-bound receiver might be to reduce compression and send more data (a highly-compressed data format might overwhelm a slow CPU more seriously than would the higher memory requirements of a lesscompressed data format), this kind of format change should be requested at the application level, not via the Slow Receiver option.

Slow Receiver implements a portion of $\mathrm{TCP}^{\prime} \mathrm{s}$ receive window functionality.

11.7. Data Dropped Option

The Data Dropped option indicates that the application data on one or more received packets did not actually reach the application. Data Dropped additionally reports why the data was dropped: perhaps the data was corrupt, or perhaps the receiver cannot keep up with the sender's current rate and the data was dropped in some receive buffer. Using Data Dropped, DCCP endpoints can discriminate between different kinds of loss; this differs from TCP, in which all loss is reported the same way.

Unless it is explicitly specified otherwise, DCCP congestion control mechanisms MUST react as if each Data Dropped packet was marked as ECN Congestion Experienced by the network. We intend for Data Dropped to enable research into richer congestion responses to corrupt and other endpoint-dropped packets, but DCCP CCIDs MUST react conservatively to Data Dropped until this behavior is standardized. Section 11.7.2, below, describes congestion responses for all current Drop Codes.

If a received packet's application data is dropped for one of the reasons listed below, this SHOULD be reported using a Data Dropped option. Alternatively, the receiver MAY choose to report as 
"received" only those packets whose data were not dropped, subject to the constraint that packets not reported as received MUST NOT have had their options processed.

The option's data looks like this:

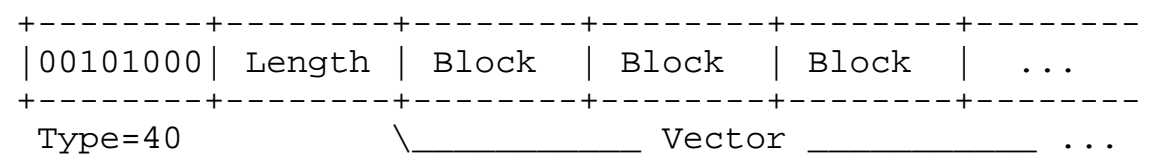

The Vector consists of a series of bytes, called Blocks, each of whose encoding corresponds to one of two choices:

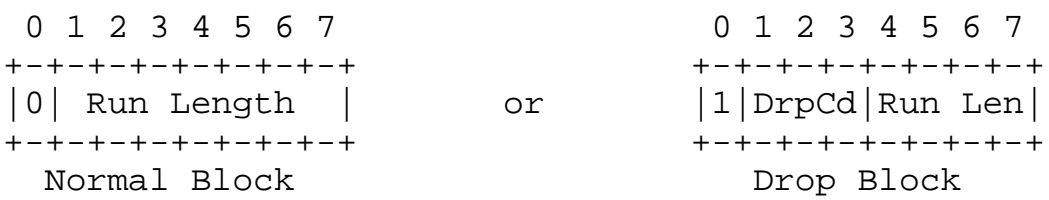

The first byte in the first Data Dropped option refers to the packet indicated by the Acknowledgement Number; subsequent bytes refer to older packets. Data Dropped MUST NOT be sent on DCCP-Data or DCCPRequest packets, which lack an Acknowledgement Number, and any Data Dropped options received on such packets MUST be ignored.

Normal Blocks, which have high bit 0 , indicate that any received packets in the Run Length had their data delivered to the application. Drop Blocks, which have high bit 1, indicate that received packets in the Run Len[gth] were not delivered as usual. The 3-bit Drop Code [DrpCd] field says what happened; generally, no data from that packet reached the application. Packets reported as "not yet received" MUST be included in Normal Blocks; packets not covered by any Data Dropped option are treated as if they were in a Normal Block. Defined Drop Codes for Drop Blocks are as follows.

$\begin{array}{cl}\text { Drop Code } & \text { Meaning } \\ 0 & \text { Protocol Constraints } \\ 1 & \text { Application Not Listening } \\ 2 & \text { Receive Buffer } \\ 3 & \text { Corrupt } \\ 4-6 & \text { Reserved } \\ 7 & \text { Delivered Corrupt }\end{array}$

Table 7: DCCP Drop Codes 
In more detail:

0 The packet data was dropped due to protocol constraints. For example, the data was included on a DCCP-Request packet, but the receiving application does not allow such piggybacking; or the data was included on a packet with inappropriately low Checksum Coverage.

1 The packet data was dropped because the application is no longer listening. See Section 11.7.2.

2 The packet data was dropped in a receive buffer, probably because of receive buffer overflow. See section 11.7.2.

3 The packet data was dropped due to corruption. See Section 9.3 .

7 The packet data was corrupted but was delivered to the application anyway. See Section 9.3.

For example, assume that a packet arrives with Acknowledgement Number 100, an Ack Vector reporting all packets as received, and a Data Dropped option containing the decimal values $0,160,3,162$. Then:

Packet 100 was received (Acknowledgement Number 100, Normal Block, Run Length 0 ).

Packet 99 was dropped in a receive buffer (Drop Block, Drop Code 2, Run Length 0).

Packets 98, 97, 96, and 95 were received (Normal Block, Run Length 3).

Packets 95, 94, and 93 were dropped in the receive buffer (Drop Block, Drop Code 2, Run Length 2).

Run lengths of more than 128 (for Normal Blocks) or 16 (for Drop Blocks) must be encoded in multiple Blocks. A single Data Dropped option can acknowledge up to 32384 Normal Block data packets, although the receiver SHOULD NOT send a Data Dropped option when all relevant packets fit into Normal Blocks. Should more packets need to be acknowledged than can fit in 253 bytes of Data Dropped, then multiple Data Dropped options can be sent. The second option will begin where the first left off, and so forth.

One or more Data Dropped options that, together, report the status of more packets than have been sent, or that change the status of a packet, or that disagree with Ack Vector or equivalent options (by 
reporting a "not yet received" packet as "dropped in the receive buffer", for example) SHOULD be considered invalid. The receiving DCCP SHOULD either ignore such options, or respond by resetting the connection with Reset Code 5, "Option Error".

A DCCP application interface should let receiving applications specify the Drop Codes corresponding to received packets. For example, this would let applications calculate their own checksums but still report "dropped due to corruption" packets via the Data Dropped option. The interface SHOULD NOT let applications reduce the "seriousness" of a packet's Drop Code; for example, the application should not be able to upgrade a packet from delivered corrupt (Drop Code 7) to delivered normally (no Drop Code).

Data Dropped information is transmitted reliably. That is, endpoints SHOULD continue to transmit Data Dropped options until receiving an acknowledgement indicating that the relevant options have been processed. In Ack Vector terms, each acknowledgement should contain Data Dropped options that cover the whole Acknowledgement Window (Section 11.4.2), although when every packet in that window would be placed in a Normal Block, no actual option is required.

11.7.1. Data Dropped and Normal Congestion Response

When deciding on a response to a particular acknowledgement or set of acknowledgements containing Data Dropped options, a congestion control mechanism MUST consider dropped packets, ECN Congestion Experienced marks (including marked packets that are included in Data Dropped), and packets singled out in Data Dropped. For window-based mechanisms, the valid response space is defined as follows.

Assume an old window of $W$. Independently calculate a new window W_new1 that assumes no packets were Data Dropped (so W_new1 contains only the normal congestion response), and a new window W_new2 that assumes no packets were lost or marked (so W_new2 contains only the Data Dropped response). We are assuming that Data Dropped recommended a reduction in congestion window, so W_new2 $<$ W.

Then the actual new window W_new MUST NOT be larger than the minimum of W_new1 and W_new2; and the sender MAY combine the two responses, by setting

$$
\mathrm{W} \_ \text {new }=W+\min \left(\mathrm{W} \_ \text {new } 1-W, 0\right)+\min \left(W_{-} \_ \text {new2 }-W, 0\right) \text {. }
$$

The details of how this is accomplished are specified in CCID profile documents. Non-window-based congestion control mechanisms MUST behave analogously; again, CCID profiles define how. 


\subsubsection{Particular Drop Codes}

Drop Code 0, Protocol Constraints, does not indicate any kind of congestion, so the sender's CCID SHOULD react to packets with Drop Code 0 as if they were received (with or without ECN Congestion Experienced marks, as appropriate). However, the sending endpoint SHOULD NOT send data until it believes the protocol constraint no longer applies.

Drop Code 1, Application Not Listening, means the application running at the endpoint that sent the option is no longer listening for data. For example, a server might close its receiving half-connection to new data after receiving a complete request from the client. This would limit the amount of state available at the server for incoming data and thus reduce the potential damage from certain denial-ofservice attacks. A Data Dropped option containing Drop Code 1 SHOULD be sent whenever received data is ignored due to a non-listening application. Once an endpoint reports Drop code 1 for a packet, it SHOULD report Drop Code 1 for every succeeding data packet on that half-connection; once an endpoint receives a Drop state 1 report, it SHOULD expect that no more data will ever be delivered to the other endpoint's application, so it SHOULD NOT send more data.

Drop Code 2, Receive Buffer, indicates congestion inside the receiving host. For instance, if a drop-from-tail kernel socket buffer is too full to accept a packet's application data, that packet should be reported as Drop Code 2. For a drop-from-head or more complex socket buffer, the dropped packet should be reported as Drop Code 2. DCCP implementations may also provide an API by which applications can mark received packets as Drop Code 2, indicating that the application ran out of space in its user-level receive buffer. (However, it is not generally useful to report packets as dropped due to Drop Code 2 after more than a couple of round-trip times have passed. The HC-Sender may have forgotten its acknowledgement state for the packet by that time, so the Data Dropped report will have no effect.) Every packet newly acknowledged as Drop Code 2 SHOULD reduce the sender's instantaneous rate by one packet per round-trip time, unless the sender is already sending one packet per RTT or less. Each CCID profile defines the CCID-specific mechanism by which this is accomplished.

Currently, the other Drop Codes (namely Drop Code 3, Corrupt; Drop Code 7, Delivered Corrupt; and reserved Drop Codes 4-6) MUST cause the relevant CCID to behave as if the relevant packets were ECN marked (ECN Congestion Experienced). 


\section{Explicit Congestion Notification}

The DCCP protocol is fully ECN-aware [RFC3168]. Each CCID specifies how its endpoints respond to ECN marks. Furthermore, DCCP, unlike TCP, allows senders to control the rate at which acknowledgements are generated (with options like Ack Ratio); since acknowledgements are congestion controlled, they also qualify as ECN-Capable Transport.

Each CCID profile describes how that CCID interacts with ECN, both for data traffic and pure-acknowledgement traffic. A sender SHOULD set ECN-Capable Transport on its packets' IP headers unless the receiver's ECN Incapable feature is on or the relevant CCID disallows it.

The rest of this section describes the ECN Incapable feature and the interaction of the ECN Nonce with acknowledgement options such as Ack Vector.

12.1. ECN Incapable Feature

DCCP endpoints are ECN-aware by default, but the ECN Incapable feature lets an endpoint reject the use of Explicit Congestion Notification. The use of this feature is NOT RECOMMENDED. ECN incapability both avoids ECN's possible benefits and prevents senders from using the ECN Nonce to check for receiver misbehavior. A DCCP stack MAY therefore leave the ECN Incapable feature unimplemented, acting as if all connections were ECN capable. Note that the inappropriate firewall interactions that dogged TCP's implementation of ECN [RFC3360] involve TCP header bits, not the IP header's ECN bits; we know of no middlebox that would block ECN-capable DCCP packets but allow ECN-incapable DCCP packets.

ECN Incapable has feature number 4 and is server-priority. It takes one-byte Boolean values. DCCP A MUST be able to read ECN bits from received frames' IP headers when ECN Incapable/A is zero. (This is independent of whether it can set ECN bits on sent frames.) DCCP A thus sends a "Change L(ECN Inapable, 1)" option to DCCP B to inform it that $A$ cannot read $E C N$ bits. If the ECN Incapable/A feature is one, then all of DCCP $B^{\prime}$ s packets MUST be sent as ECN incapable. New connections start with ECN Incapable 0 (that is, ECN capable) for both endpoints. Values of two or more are reserved.

If a DCCP is not ECN capable, it MUST send Mandatory "Change L (ECN Incapable, 1)" options to the other endpoint until acknowledged (by "Confirm R(ECN Incapable, 1)") or the connection closes. Furthermore, it MUST NOT accept any data until the other endpoint 
sends "Confirm R(ECN Incapable, 1)". It SHOULD send Data Dropped options on its acknowledgements, with Drop Code 0 ("protocol constraints"), if the other endpoint does send data inappropriately.

\subsection{ECN Nonces}

Congestion avoidance will not occur, and the receiver will sometimes get its data faster, if the sender isn't told about congestion events. Thus, the receiver has some incentive to falsify acknowledgement information, reporting that marked or dropped packets were actually received unmarked. This problem is more serious with DCCP than with TCP, since TCP provides reliable transport: it is more difficult with TCP to lie about lost packets without breaking the application.

ECN Nonces are a general mechanism to prevent ECN cheating (or loss cheating). Two values for the two-bit ECN header field indicate ECN-Capable Transport, 01 and 10. The second code point, 10, is the ECN Nonce. In general, a protocol sender chooses between these code points randomly on its output packets, remembering the sequence it chose. On every acknowledgement, the protocol receiver reports the number of ECN Nonces it has received thus far. This is called the ECN Nonce Echo. Since ECN marking and packet dropping both destroy the ECN Nonce, a receiver that lies about an ECN mark or packet drop has a 50\% chance of guessing right and avoiding discipline. The sender may react punitively to an ECN Nonce mismatch, possibly up to dropping the connection. The ECN Nonce Echo field need not be an integer; one bit is enough to catch 50\% of infractions, and the probability of success drops exponentially as more packets are sent [RFC3540].

In DCCP, the ECN Nonce Echo field is encoded in acknowledgement options. For example, the Ack Vector option comes in two forms, Ack Vector [Nonce 0] (option 38) and Ack Vector [Nonce 1] (option 39), corresponding to the two values for a one-bit ECN Nonce Echo. The Nonce Echo for a given Ack Vector equals the one-bit sum (exclusiveor, or parity) of ECN nonces for packets reported by that Ack Vector as received and not ECN marked. Thus, only packets marked as state 0 matter for this calculation (that is, valid received packets that were not ECN marked). Every Ack Vector option is detailed enough for the sender to determine what the Nonce Echo should have been. It can check this calculation against the actual Nonce Echo and complain if there is a mismatch. (The Ack Vector could conceivably report every packet's ECN Nonce state, but this would severely limit its compressibility without providing much extra protection.)

Each DCCP sender SHOULD set ECN Nonces on its packets and remember which packets had nonces. When a sender detects an ECN Nonce Echo 
mismatch, it behaves as described in the next section. Each DCCP receiver MUST calculate and use the correct value for ECN Nonce Echo when sending acknowledgement options.

ECN incapability, as indicated by the ECN Incapable feature, is handled as follows: an endpoint sending packets to an ECN-incapable receiver MUST send its packets as ECN incapable, and an ECNincapable receiver MUST use the value zero for all ECN Nonce Echoes.

\subsection{Aggression Penalties}

DCCP endpoints have several mechanisms for detecting congestionrelated misbehavior. For example:

- A sender can detect an ECN Nonce Echo mismatch, indicating possible receiver misbehavior.

- A receiver can detect whether the sender is responding to congestion feedback or Slow Receiver.

- An endpoint may be able to detect that its peer is reporting inappropriately small Elapsed Time values (Section 13.2).

An endpoint that detects possible congestion-related misbehavior SHOULD try to verify that its peer is truly misbehaving. For example, a sending endpoint might send a packet whose ECN header field is set to Congestion Experienced, 11; a receiver that doesn't report a corresponding mark is most likely misbehaving.

Upon detecting possible misbehavior, a sender sHould respond as if the receiver had reported one or more recent packets as ECN-marked (instead of unmarked), while a receiver SHOULD report one or more recent non-marked packets as ECN-marked. Alternately, a sender might act as if the receiver had sent a Slow Receiver option, and a receiver might send Slow Receiver options. Other reactions that serve to slow the transfer rate are also acceptable. An entity that detects particularly egregious and ongoing misbehavior MAY also reset the connection with Reset Code 11, "Aggression Penalty".

However, ECN Nonce mismatches and other warning signs can result from innocent causes, such as implementation bugs or attack. In particular, a successful DCCP-Data attack (Section 7.5.5) can cause the receiver to report an incorrect ECN Nonce Echo. Therefore, connection reset and other heavyweight mechanisms SHOULD be used only as last resorts, after multiple round-trip times of verified aggression. 


\section{Timing Options}

The Timestamp, Timestamp Echo, and Elapsed Time options help DCCP endpoints explicitly measure round-trip times.

\subsection{Timestamp Option}

This option is permitted in any DCCP packet. The length of the option is 6 bytes.

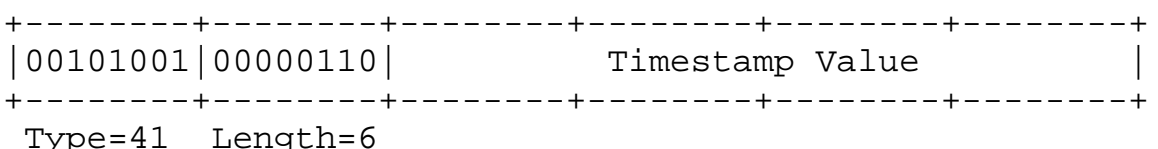

The four bytes of option data carry the timestamp of this packet. The timestamp is a 32-bit integer that increases monotonically with time, at a rate of 1 unit per 10 microseconds. At this rate, Timestamp Value will wrap approximately every 11.9 hours. Endpoints need not measure time at this fine granularity; for example, an endpoint that preferred to measure time at millisecond granularity might send Timestamp Values that were all multiples of 100 . The precise time corresponding to Timestamp Value zero is not specified: Timestamp Values are only meaningful relative to other Timestamp Values sent on the same connection. A DCCP receiving a Timestamp option SHOULD respond with a Timestamp Echo option on the next packet it sends.

\subsection{Elapsed Time Option}

This option is permitted in any DCCP packet that contains an Acknowledgement Number; such options received on other packet types MUST be ignored. It indicates how much time has elapsed since the packet being acknowledged -- the packet with the given Acknowledgement Number -- was received. The option may take 4 or 6 bytes, depending on the size of the Elapsed Time value. Elapsed Time helps correct round-trip time estimates when the gap between receiving a packet and acknowledging that packet may be long -- in CCID 3, for example, where acknowledgements are sent infrequently. 


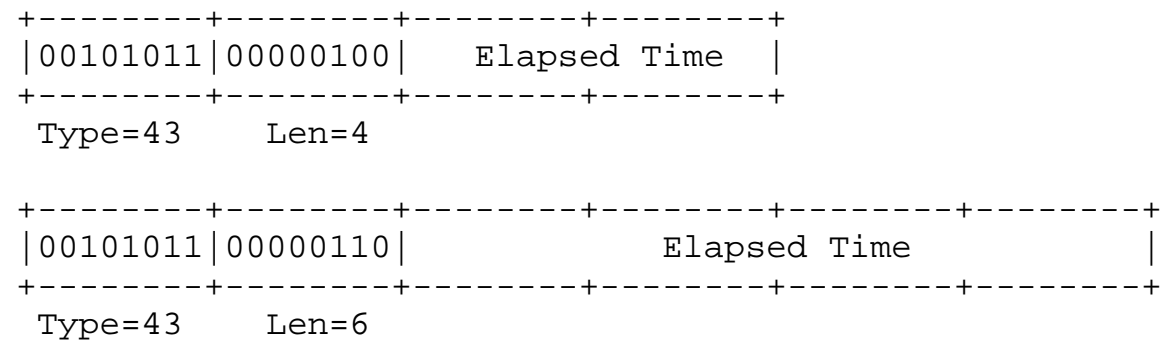

The option data, Elapsed Time, represents an estimated lower bound on the amount of time elapsed since the packet being acknowledged was received, with units of hundredths of milliseconds. If Elapsed Time is less than a half-second, the first, smaller form of the option SHOULD be used. Elapsed Times of more than 0.65535 seconds MUST be sent using the second form of the option. The special Elapsed Time value 4294967295, which corresponds to approximately 11.9 hours, is used to represent any Elapsed Time greater than 42949.67294 seconds. DCCP endpoints MUST NOT report Elapsed Times that are significantly larger than the true elapsed times. A connection MAY be reset with Reset Code 11, "Aggression Penalty", if one endpoint determines that the other is reporting a much-too-large Elapsed Time.

Elapsed Time is measured in hundredths of milliseconds as a compromise between two conflicting goals. First, it provides enough granularity to reduce rounding error when measuring elapsed time over fast LANs; second, it allows many reasonable elapsed times to fit into two bytes of data.

\subsection{Timestamp Echo Option}

This option is permitted in any DCCP packet, as long as at least one packet carrying the Timestamp option has been received. Generally, a DCCP endpoint should send one Timestamp Echo option for each Timestamp option it receives, and it should send that option as soon as is convenient. The length of the option is between 6 and 10 bytes, depending on whether Elapsed Time is included and how large it is. 

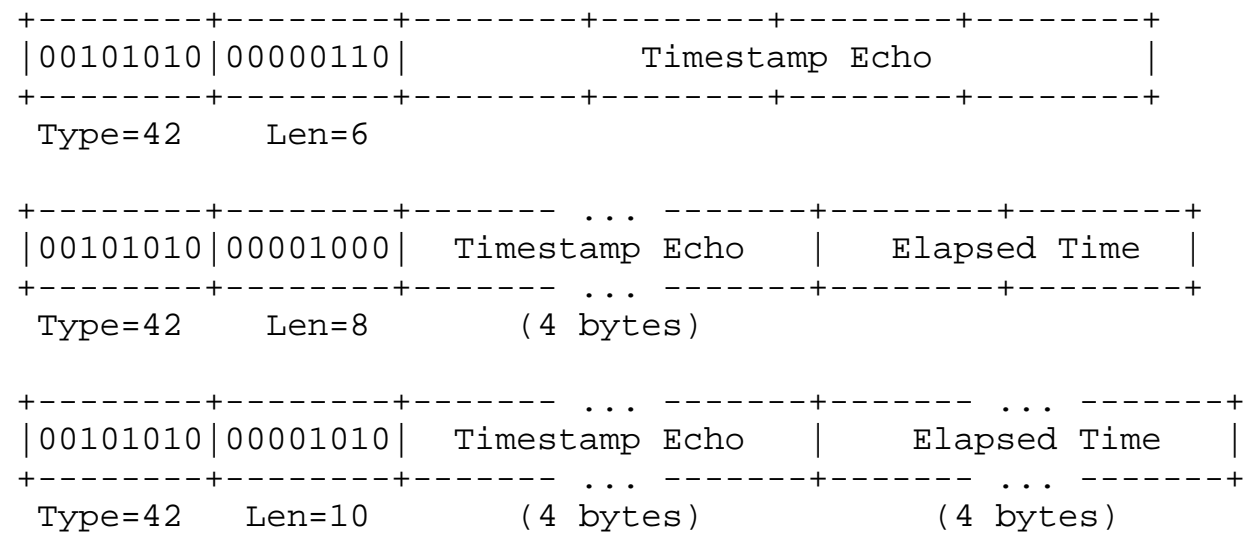

The first four bytes of option data, Timestamp Echo, carry a Timestamp Value taken from a preceding received Timestamp option. Usually, this will be the last packet that was received -- the packet indicated by the Acknowledgement Number, if any -- but it might be a preceding packet. Each Timestamp received will generally result in exactly one Timestamp Echo transmitted. If an endpoint has received multiple Timestamp options since the last time it sent a packet, then it MAY ignore all Timestamp options but the one included on the packet with the greatest sequence number. Alternatively, it MAY include multiple Timestamp Echo options in its response, each corresponding to a different Timestamp option.

The Elapsed Time value, similar to that in the Elapsed Time option, indicates the amount of time elapsed since receiving the packet whose timestamp is being echoed. This time MUST have units of hundredths of milliseconds. Elapsed Time is meant to help the Timestamp sender separate the network round-trip time from the Timestamp receiver's processing time. This may be particularly important for CCIDs where acknowledgements are sent infrequently, so that there might be considerable delay between receiving a Timestamp option and sending the corresponding Timestamp Echo. A missing Elapsed Time field is equivalent to an Elapsed Time of zero. The smallest version of the option SHOULD be used that can hold the relevant Elapsed Time value.

\section{Maximum Packet Size}

A DCCP implementation MUST maintain the maximum packet size (MPS) allowed for each active DCCP session. The MPS is influenced by the maximum packet size allowed by the current congestion control mechanism (CCMPS), the maximum packet size supported by the path's links (PMTU, the Path Maximum Transmission Unit) [RFC1191], and the lengths of the IP and DCCP headers. 
A DCCP application interface SHOULD let the application discover DCCP's current MPS. Generally, the DCCP implementation will refuse to send any packet bigger than the MPS, returning an appropriate error to the application. A DCCP interface MAY allow applications to request fragmentation for packets larger than PMTU, but not larger than CCMPS. (Packets larger than CCMPS MUST be rejected in any case.) Fragmentation SHOULD NOT be the default, since it decreases robustness: an entire packet is discarded if even one of its fragments is lost. Applications can usually get better error tolerance by producing packets smaller than the PMTU.

The MPS reported to the application SHOULD be influenced by the size expected to be required for DCCP headers and options. If the application provides data that, when combined with the options the DCCP implementation would like to include, would exceed the MPS, the implementation should either send the options on a separate packet (such as a DCCP-Ack) or lower the MPS, drop the data, and return an appropriate error to the application.

\subsection{Measuring PMTU}

Each DCCP endpoint MUST keep track of the current PMTU for each connection, except that this is not required for IPv4 connections whose applications have requested fragmentation. The PMTU SHOULD be initialized from the interface MTU that will be used to send packets. The MPS will be initialized with the minimum of the PMTU and the CCMPS, if any.

Classical PMTU discovery uses unfragmentable packets. In IPv4, these packets have the IP Don't Fragment (DF) bit set; in IPv6, all packets are unfragmentable once emitted by an end host. As specified in [RFC1191], when a router receives a packet with DF set that is larger than the next link's MTU, it sends an ICMP Destination Unreachable message back to the source whose Code indicates that an unfragmentable packet was too large to forward (a "Datagram Too Big" message). When a DCCP implementation receives a Datagram Too Big message, it decreases its PMTU to the Next-Hop MTU value given in the ICMP message. If the MTU given in the message is zero, the sender chooses a value for PMTU using the algorithm described in [RFC1191], Section 7. If the MTU given in the message is greater than the current PMTU, the Datagram Too Big message is ignored, as described in [RFC1191]. (We are aware that this may cause problems for DCCP endpoints behind certain firewalls.)

A DCCP implementation may allow the application occasionally to request that PMTU discovery be performed again. This will reset the PMTU to the outgoing interface's MTU. Such requests SHOULD be rate limited, to one per two seconds, for example. 
A DCCP sender MAY treat the reception of an ICMP Datagram Too Big message as an indication that the packet being reported was not lost due to congestion, and so for the purposes of congestion control it MAY ignore the DCCP receiver's indication that this packet did not arrive. However, if this is done, then the DCCP sender MUST check the ECN bits of the IP header echoed in the ICMP message and only perform this optimization if these ECN bits indicate that the packet did not experience congestion prior to reaching the router whose link MTU it exceeded.

A DCCP implementation SHOULD ensure, as far as possible, that ICMP Datagram Too Big messages were actually generated by routers, so that attackers cannot drive the PMTU down to a falsely small value. The simplest way to do this is to verify that the sequence Number on the ICMP error's encapsulated header corresponds to a sequence Number that the implementation recently sent. (According to current specifications, routers should return the full DCCP header and payload up to a maximum of 576 bytes [RFC1812] or the minimum IPv6 MTU [RFC2463], although they are not required to return more than 64 bits [RFC792]. Any amount greater than 128 bits will include the Sequence Number.) ICMP Datagram Too Big messages with incorrect or missing Sequence Numbers may be ignored, or the DCCP implementation may lower the PMTU only temporarily in response. If more than three odd Datagram Too Big messages are received and the other DCCP endpoint reports more than three lost packets, however, the DCCP implementation SHOULD assume the presence of a confused router and either obey the ICMP messages' PMTU or (on IPV4 networks) switch to allowing fragmentation.

DCCP also allows upward probing of the PMTU [PMTUD], where the DCCP endpoint begins by sending small packets with DF set and then gradually increases the packet size until a packet is lost. This mechanism does not require any ICMP error processing. DCCP-Sync packets are the best choice for upward probing, since DCCP-Sync probes do not risk application data loss. The DCCP implementation inserts arbitrary data into the DCCP-Sync application area, padding the packet to the right length. Since every valid DCCP-Sync generates an immediate DCCP-SyncAck in response, the endpoint will have a pretty good idea of when a probe is lost.

\subsection{Sender Behavior}

A DCCP sender SHOULD send every packet as unfragmentable, as described above, with the following exceptions.

- On IPv4 connections whose applications have requested fragmentation, the sender SHOULD send packets with the DF bit not set. 
- On IPv6 connections whose applications have requested fragmentation, the sender SHOULD use fragmentation extension headers to fragment packets larger than PMTU into suitably-sized chunks. (Those chunks are, of course, unfragmentable.)

- It is undesirable for PMTU discovery to occur on the initial connection setup handshake, as the connection setup process may not be representative of packet sizes used during the connection, and performing MTU discovery on the initial handshake might unnecessarily delay connection establishment. Thus, DCCP-Request and DCCP-Response packets SHOULD be sent as fragmentable. In addition, DCCP-Reset packets SHOULD be sent as fragmentable, although typically these would be small enough to not be a problem. For IPv4 connections, these packets SHOULD be sent with the DF bit not set; for IPv6 connections, they SHOULD be preemptively fragmented to a size not larger than the relevant interface MTU.

If the DCCP implementation has decreased the PMTU, the sending application has not requested fragmentation, and the sending application attempts to send a packet larger than the new MPS, the API MUST refuse to send the packet and return an appropriate error to the application. The application should then use the API to query the new value of MPS. The kernel might have some packets buffered for transmission that are smaller than the old MPS but larger than the new MPS. It MAY send these packets as fragmentable, or it MAY discard these packets; it MUST NOT send them as unfragmentable.

\section{Forward Compatibility}

Future versions of DCCP may add new options and features. A few simple guidelines will let extended DCCPs interoperate with normal DCCPs .

- DCCP processors MUST NOT act punitively towards options and features they do not understand. For example, DCCP processors MUST NOT reset the connection if some field marked Reserved in this specification is non-zero; if some unknown option is present; or if some feature negotiation option mentions an unknown feature. Instead, DCCP processors MUST ignore these events. The Mandatory option is the single exception: if Mandatory precedes some unknown option or feature, the connection MUST be reset.

- DCCP processors MUST anticipate the possibility of unknown feature values, which might occur as part of a negotiation for a known feature. For server-priority features, unknown values are handled as a matter of course: since the non-extended DCCP's priority list will not contain unknown values, the result of the negotiation 
cannot be an unknown value. A DCCP MUST respond with an empty Confirm option if it is assigned an unacceptable value for some non-negotiable feature.

- Each DCCP extension SHOULD be controlled by some feature. The default value of this feature SHOULD correspond to "extension not available". If an extended DCCP wants to use the extension, it SHOULD attempt to change the feature's value using a change L or Change $\mathrm{R}$ option. Any non-extended DCCP will ignore the option, thus leaving the feature value at its default, "extension not available".

Section 19 lists DCCP assigned numbers reserved for experimental and testing purposes.

16. Middlebox Considerations

This section describes properties of DCCP that firewalls, network address translators, and other middleboxes should consider, including parts of the packet that middleboxes should not change. The intent is to draw attention to aspects of DCCP that may be useful, or dangerous, for middleboxes, or that differ significantly from TCP.

The Service Code field in DCCP-Request packets provides information that may be useful for stateful middleboxes. With Service Code, a middlebox can tell what protocol a connection will use without relying on port numbers. Middleboxes can disallow connections that attempt to access unexpected services by sending a DCCP-Reset with Reset Code 8, "Bad Service Code". Middleboxes should not modify the Service Code unless they are really changing the service a connection is accessing.

The Source and Destination Port fields are in the same packet locations as the corresponding fields in TCP and UDP, which may simplify some middlebox implementations.

The forward compatibility considerations in Section 15 apply to middleboxes as well. In particular, middleboxes generally shouldn't act punitively towards options and features they do not understand.

Modifying DCCP Sequence Numbers and Acknowledgement Numbers is more tedious and dangerous than modifying TCP sequence numbers. A middlebox that added packets to or removed packets from a DCCP connection would have to modify acknowledgement options, such as Ack Vector, and CCID-specific options, such as TFRC's Loss Intervals, at minimum. On ECN-capable connections, the middlebox would have to keep track of ECN Nonce information for packets it introduced or removed, so that the relevant acknowledgement options continued to 
have correct ECN Nonce Echoes, or risk the connection being reset for "Aggression Penalty". We therefore recommend that middleboxes not modify packet streams by adding or removing packets.

Note that there is less need to modify DCCP's per-packet sequence numbers than to modify $\mathrm{TCP}^{\prime} \mathrm{s}$ per-byte sequence numbers; for example, a middlebox can change the contents of a packet without changing its sequence number. (In TCP, sequence number modification is required to support protocols like FTP that carry variable-length addresses in the data stream. If such an application were deployed over DCCP, middleboxes would simply grow or shrink the relevant packets as necessary without changing their sequence numbers. This might involve fragmenting the packet.)

Middleboxes may, of course, reset connections in progress. Clearly, this requires inserting a packet into one or both packet streams, but the difficult issues do not arise.

DCCP is somewhat unfriendly to "connection splicing" [SHHP00], in which clients' connection attempts are intercepted, but possibly later "spliced in" to external server connections via sequence number manipulations. A connection splicer at minimum would have to ensure that the spliced connections agreed on all relevant feature values, which might take some renegotiation.

The contents of this section should not be interpreted as a wholesale endorsement of stateful middleboxes.

17. Relations to Other Specifications

17.1. $\operatorname{RTP}$

The Real-Time Transport Protocol, RTP [RFC3550], is currently used over UDP by many of DCCP's target applications (for instance, streaming media). Therefore, it is important to examine the relationship between DCCP and RTP and, in particular, the question of whether any changes in RTP are necessary or desirable when it is layered over DCCP instead of UDP.

There are two potential sources of overhead in the RTP-over-DCCP combination: duplicated acknowledgement information and duplicated sequence numbers. Together, these sources of overhead add slightly more than 4 bytes per packet relative to RTP-over-UDP, and eliminating the redundancy would not reduce the overhead.

First, consider acknowledgements. Both RTP and DCCP report feedback about loss rates to data senders, via RTP Control Protocol sender and Receiver Reports (RTCP SR/RR packets) and via DCCP acknowledgement 
options. These feedback mechanisms are potentially redundant. However, RTCP SR/RR packets contain information not present in DCCP acknowledgements, such as "interarrival jitter", and DCCP's acknowledgements contain information not transmitted by RTCP, such as the ECN Nonce Echo. Neither feedback mechanism makes the other redundant.

Sending both types of feedback need not be particularly costly either. RTCP reports may be sent relatively infrequently: once every 5 seconds on average, for low-bandwidth flows. In DCCP, some feedback mechanisms are expensive -- Ack Vector, for example, is frequent and verbose -- but others are relatively cheap: CCID 3 (TFRC) acknowledgements take between 16 and 32 bytes of options sent once per round-trip time. (Reporting less frequently than once per RTT would make congestion control less responsive to loss.) We therefore conclude that acknowledgement overhead in RTP-over-DCCP need not be significantly higher than for RTP-over-UDP, at least for CCID 3 .

One clear redundancy can be addressed at the application level. The verbose packet-by-packet loss reports sent in RTCP Extended Reports Loss RLE Blocks [RFC3611] can be derived from DCCP's Ack Vector options. (The converse is not true, since Loss RLE Blocks contain no ECN information.) Since DCCP implementations should provide an API for application access to Ack Vector information, RTP-over-DCCP applications might request either DCCP Ack Vectors or RTCP Extended Report Loss RLE Blocks, but not both.

Now consider sequence number redundancy on data packets. The embedded RTP header contains a 16-bit RTP sequence number. Most data packets will use the DCCP-Data type; DCCP-DataAck and DCCP-ACk packets need not usually be sent. The DCCP-Data header is 12 bytes long without options, including a 24-bit sequence number. This is 4 bytes more than a UDP header. Any options required on data packets would add further overhead, although many CCIDs (for instance, CCID 3, TFRC) don't require options on most data packets.

The DCCP sequence number cannot be inferred from the RTP sequence number since it increments on non-data packets as well as data packets. The RTP sequence number cannot be inferred from the DCCP sequence number either [RFC3550]. Furthermore, removing RTP's sequence number would not save any header space because of alignment issues. We therefore recommend that RTP transmitted over DCCP use the same headers currently defined. The 4 byte header cost is a reasonable tradeoff for $\mathrm{DCCP}^{\prime} \mathrm{s}$ congestion control features and access to ECN. Truly bandwidth-starved endpoints should use some header compression scheme. 


\subsection{Congestion Manager and Multiplexing}

Since DCCP doesn't provide reliable, ordered delivery, multiple application sub-flows may be multiplexed over a single DCCP connection with no inherent performance penalty. Thus, there is no need for DCCP to provide built-in support for multiple sub-flows. This differs from SCTP [RFC2960].

Some applications might want to share congestion control state among multiple DCCP flows that share the same source and destination addresses. This functionality could be provided by the Congestion Manager [RFC3124], a generic multiplexing facility. However, the CM would not fully support DCCP without change; it does not gracefully handle multiple congestion control mechanisms, for example.

\section{Security Considerations}

DCCP does not provide cryptographic security guarantees. Applications desiring cryptographic security services (integrity, authentication, confidentiality, access control, and anti-replay protection) should use IPsec or end-to-end security of some kind; Secure RTP is one candidate protocol [RFC3711].

Nevertheless, DCCP is intended to protect against some classes of attackers: Attackers cannot hijack a DCCP connection (close the connection unexpectedly, or cause attacker data to be accepted by an endpoint as if it came from the sender) unless they can guess valid sequence numbers. Thus, as long as endpoints choose initial sequence numbers well, a DCCP attacker must snoop on data packets to get any reasonable probability of success. Sequence number validity checks provide this guarantee. Section 7.5.5 describes sequence number security further. This security property only holds assuming that DCCP's random numbers are chosen according to the guidelines in [RFC4086].

DCCP also provides mechanisms to limit the potential impact of some denial-of-service attacks. These mechanisms include Init cookie (Section 8.1.4), the DCCP-CloseReq packet (Section 5.5), the Application Not Listening Drop Code (Section 11.7.2), limitations on the processing of options that might cause connection reset (Section 7.5.5), limitations on the processing of some ICMP messages (Section 14.1), and various rate limits, which let servers avoid extensive computation or packet generation (Sections 7.5.3, 8.1.3, and others).

DCCP provides no protection against attackers that can snoop on data packets. 


\subsection{Security Considerations for Partial Checksums}

The partial checksum facility has a separate security impact, particularly in its interaction with authentication and encryption mechanisms. The impact is the same in DCCP as in the UDP-Lite protocol, and what follows was adapted from the corresponding text in the UDP-Lite specification [RFC3828].

When a DCCP packet's Checksum Coverage field is not zero, the uncovered portion of a packet may change in transit. This is contrary to the idea behind most authentication mechanisms: authentication succeeds if the packet has not changed in transit. Unless authentication mechanisms that operate only on the sensitive part of packets are developed and used, authentication will always fail for partially-checksummed DCCP packets whose uncovered part has been damaged.

The IPsec integrity check (Encapsulation Security Protocol, ESP, or Authentication Header, $\mathrm{AH}$ ) is applied (at least) to the entire IP packet payload. Corruption of any bit within that area will then result in the IP receiver's discarding a DCCP packet, even if the corruption happened in an uncovered part of the DCCP application data.

When IPsec is used with ESP payload encryption, a link can not determine the specific transport protocol of a packet being forwarded by inspecting the IP packet payload. In this case, the link MUST provide a standard integrity check covering the entire IP packet and payload. DCCP partial checksums provide no benefit in this case.

Encryption (e.g., at the transport or application levels) may be used. Note that omitting an integrity check can, under certain circumstances, compromise confidentiality [B98].

If a few bits of an encrypted packet are damaged, the decryption transform will typically spread errors so that the packet becomes too damaged to be of use. Many encryption transforms today exhibit this behavior. There exist encryption transforms, stream ciphers, that do not cause error propagation. Proper use of stream ciphers can be quite difficult, especially when authentication checking is omitted [BB01]. In particular, an attacker can cause predictable changes to the ultimate plaintext, even without being able to decrypt the ciphertext. 


\section{IANA Considerations}

IANA has assigned IP Protocol Number 33 to DCCP.

DCCP introduces eight sets of numbers whose values should be allocated by IANA. We refer to allocation policies, such as Standards Action, outlined in [RFC2434], and most registries reserve some values for experimental and testing use [RFC3692]. In addition, DCCP requires that the IANA Port Numbers registry be opened for DCCP port registrations; Section 19.9 describes how. The IANA should feel free to contact the DCCP Expert Reviewer with questions on any registry, regardless of the registry policy, for clarification or if there is a problem with a request.

19.1. Packet Types Registry

Each entry in the DCCP Packet Types registry contains a packet type, which is a number in the range 0-15; a packet type name, such as DCCP-Request; and a reference to the RFC defining the packet type. The registry is initially populated using the values in Table 1 (Section 5.1). This document allocates packet types 0-9, and packet type 14 is permanently reserved for experimental and testing use. Packet types 10-13 and 15 are currently reserved and should be allocated with the Standards Action policy, which requires IESG review and approval and standards-track IETF RFC publication.

\subsection{Reset Codes Registry}

Each entry in the DCCP Reset Codes registry contains a Reset Code, which is a number in the range 0-255; a short description of the Reset Code, such as "No Connection"; and a reference to the RFC defining the Reset Code. The registry is initially populated using the values in Table 2 (Section 5.6). This document allocates Reset Codes 0-11, and Reset Codes 120-126 are permanently reserved for experimental and testing use. Reset Codes $12-119$ and 127 are currently reserved and should be allocated with the IETF Consensus policy, requiring an IETF RFC publication (standards track or not) with IESG review and approval. Reset Codes 128-255 are permanently reserved for CCID-specific registries; each CCID Profile document describes how the corresponding registry is managed.

\subsection{Option Types Registry}

Each entry in the DCCP option types registry contains an option type, which is a number in the range 0-255; the name of the option, such as "Slow Receiver"; and a reference to the RFC defining the option type. The registry is initially populated using the values in Table 3

(Section 5.8). This document allocates option types 0-2 and 32-44, 
and option types 31 and 120-126 are permanently reserved for experimental and testing use. Option types 3-30, 45-119, and 127 are currently reserved and should be allocated with the IETF Consensus policy, requiring an IETF RFC publication (standards track or not) with IESG review and approval. Option types 128-255 are permanently reserved for CCID-specific registries; each CCID Profile document describes how the corresponding registry is managed.

\subsection{Feature Numbers Registry}

Each entry in the DCCP feature numbers registry contains a feature number, which is a number in the range 0-255; the name of the feature, such as "ECN Incapable"; and a reference to the RFC defining the feature number. The registry is initially populated using the values in Table 4 (Section 6). This document allocates feature numbers 0-9, and feature numbers 120-126 are permanently reserved for experimental and testing use. Feature numbers 10-119 and 127 are currently reserved and should be allocated with the IETF Consensus policy, requiring an IETF RFC publication (standards track or not) with IESG review and approval. Feature numbers 128-255 are permanently reserved for CCID-specific registries; each CCID Profile document describes how the corresponding registry is managed.

\subsection{Congestion Control Identifiers Registry}

Each entry in the DCCP Congestion Control Identifiers (CCIDs) registry contains a CCID, which is a number in the range 0-255; the name of the CCID, such as "TCP-like Congestion Control"; and a reference to the RFC defining the CCID. The registry is initially populated using the values in Table 5 (Section 10). CCIDs 2 and 3 are allocated by concurrently published profiles, and CCIDs 248-254 are permanently reserved for experimental and testing use. CCIDs 0 , 1, 4-247, and 255 are currently reserved and should be allocated with the IETF Consensus policy, requiring an IETF RFC publication

(standards track or not) with IESG review and approval.

\subsection{Ack Vector States Registry}

Each entry in the DCCP Ack Vector States registry contains an Ack Vector State, which is a number in the range 0-3; the name of the State, such as "Received ECN Marked"; and a reference to the RFC defining the state. The registry is initially populated using the values in Table 6 (Section 11.4). This document allocates states 0 , 1 , and 3 . State 2 is currently reserved and should be allocated with the Standards Action policy, which requires IESG review and approval and standards-track IETF RFC publication. 


\subsection{Drop Codes Registry}

Each entry in the DCCP Drop Codes registry contains a Data Dropped Drop Code, which is a number in the range 0-7; the name of the Drop Code, such as "Application Not Listening"; and a reference to the RFC defining the Drop Code. The registry is initially populated using the values in Table 7 (Section 11.7). This document allocates Drop Codes 0-3 and 7. Drop Codes 4-6 are currently reserved, and should be allocated with the Standards Action policy, which requires IESG review and approval and standards-track IETF RFC publication.

\subsection{Service Codes Registry}

Each entry in the Service Codes registry contains a Service Code, which is a number in the range 0-4294967294; a short English description of the intended service; and an optional reference to an RFC or other publicly available specification defining the Service code. The registry should list the Service Code's numeric value as a decimal number. When the Service Code may be represented in "SC:" format according to the rules in section 8.1.2, the registry should also show the corresponding ASCII interpretation of the Service Code minus the "SC:" prefix. Thus, the number 1717858426 would additionally appear as "fdpz". Service Codes are not DCCP-specific. Service Code 0 is permanently reserved (it represents the absence of a meaningful Service Code), and Service Codes 1056964608-1073741823 (high byte ASCII "?") are reserved for Private Use. Note that 4294967295 is not a valid Service Code. Most of the remaining Service Codes are allocated First Come First Served, with no RFC publication required; exceptions are listed in section 8.1.2. This document allocates a single Service Code, 1145656131 ("DISC"). This corresponds to the discard service, which discards all data sent to the service and sends no data in reply.

\subsection{Port Numbers Registry}

DCCP services may use contact port numbers to provide service to unknown callers, as in TCP and UDP. IANA is therefore requested to open the existing Port Numbers registry for DCCP using the following rules, which we intend to mesh well with existing Port Numbers registration procedures.

Port numbers are divided into three ranges. The Well known Ports are those from 0 through 1023, the Registered Ports are those from 1024 through 49151, and the Dynamic and/or Private Ports are those from 49152 through 65535. Well known and Registered Ports are intended for use by server applications that desire a default contact point on a system. On most systems, Well known ports can only be used by system (or root) processes or by programs executed by privileged 
users, while Registered Ports can be used by ordinary user processes or programs executed by ordinary users. Dynamic and/or Private Ports are intended for temporary use, including client-side ports, out-ofband negotiated ports, and application testing prior to registration of a dedicated port; they MUST NOT be registered.

The Port Numbers registry should accept registrations for DCCP ports in the Well Known Ports and Registered Ports ranges. Well Known and Registered Ports SHOULD NOT be used without registration. Although in some cases -- such as porting an application from UDP to DCCP -it may seem natural to use a DCCP port before registration completes, we emphasize that IANA will not guarantee registration of particular Well Known and Registered Ports. Registrations should be requested as early as possible.

Each port registration SHALL include the following information:

- A short port name, consisting entirely of letters ( $A-z$ and $a-z)$, digits (0-9), and punctuation characters from "-_+./*" (not including the quotes).

o The port number that is requested to be registered.

- A short English phrase describing the port's purpose. This MUST include one or more space-separated textual Service Code descriptors naming the port's corresponding Service Codes (see Section 8.1.2).

- Name and contact information for the person or entity performing the registration, and possibly a reference to a document defining the port's use. Registrations coming from IETF working groups need only name the working group, but indicating a contact person is recommended.

Registrants are encouraged to follow these guidelines when submitting a registration.

- A port name SHOULD NOT be registered for more than one DCCP port number.

- A port name registered for UDP MAY be registered for DCCP as well. Any such registration SHOULD use the same port number as the existing UDP registration.

- Concrete intent to use a port SHOULD precede port registration. For example, existing UDP ports SHOULD NOT be registered in advance of any intent to use those ports for DCCP. 
- A port name generally associated with TCP and/or SCTP SHOULD NOT be registered for DCCP, since that port name implies reliable transport. For example, we discourage registration of any "http" port for DCCP. However, if such a registration makes sense (that is, if there is concrete intent to use such a port), the DCCP registration SHOULD use the same port number as the existing registration.

- Multiple DCCP registrations for the same port number are allowed as long as the registrations' Service Codes do not overlap.

This document registers the following port. (This should be considered a model registration.)

discard 9/dccp Discard SC:DISC

\# IETF dccp WG, Eddie Kohler <kohler@cs.ucla.edu>, [RFC4340]

The discard service, which accepts DCCP connections on port 9, discards all incoming application data and sends no data in response. Thus, DCCP's discard port is analogous to TCP's discard port, and might be used to check the health of a DCCP stack.

20. Thanks

Thanks to Jitendra Padhye for his help with early versions of this specification.

Thanks to Junwen Lai and Arun Venkataramani, who, as interns at ICIR, built a prototype DCCP implementation. In particular, Junwen Lai recommended that the old feature negotiation mechanism be scrapped and co-designed the current mechanism. Arun Venkataramani's feedback improved Appendix A.

We thank the staff and interns of ICIR and, formerly, ACIRI, the members of the End-to-End Research Group, and the members of the Transport Area Working Group for their feedback on DCCP. We especially thank the DCCP expert reviewers Greg Minshall, Eric Rescorla, and Magnus Westerlund for detailed written comments and problem spotting, and Rob Austein and Steve Bellovin for verbal comments and written notes. We also especially thank Aaron Falk, the working group chair during the development of this specification.

We also thank those who provided comments and suggestions via the DCCP BOF, Working Group, and mailing lists, including Damon Lanphear, Patrick McManus, Colin Perkins, Sara Karlberg, Kevin Lai, Bernard Aboba, Youngsoo Choi, Pengfei Di, Dan Duchamp, Lars Eggert, Gorry Fairhurst, Derek Fawcus, David Timothy Fleeman, John Loughney, Ghyslain Pelletier, Hagen Paul Pfeifer, Tom Phelan, Stanislav 
Shalunov, Somsak Vanit-Anunchai, David Vos, Yufei Wang, and Michael Welzl. In particular, Colin Perkins provided extensive, detailed feedback, Michael Welzl suggested the Data Checksum option, Gorry Fairhurst provided extensive feedback on various checksum issues, and Somsak Vanit-Anunchai, Jonathan Billington, and Tul Kongprakaiwoot's Colored Petri Net model [VBK05] discovered several problems with message exchange. 


\section{A. Appendix: Ack Vector Implementation Notes}

This appendix discusses particulars of DCCP acknowledgement handling in the context of an abstract implementation for Ack Vector. It is informative and not normative.

The first part of our implementation runs at the HC-Receiver, and therefore acknowledges data packets. It generates Ack Vector options. The implementation has the following characteristics:

- At most one byte of state per acknowledged packet.

- $O(1)$ time to update that state when a new packet arrives (normal case).

- Cumulative acknowledgements.

- Quick removal of old state.

The basic data structure is a circular buffer containing information about acknowledged packets. Each byte in this buffer contains a state and run length; the state can be 0 (packet received), 1 (packet ECN marked), or 3 (packet not yet received). The buffer grows from right to left. The implementation maintains five variables, aside from the buffer contents:

o "buf_head" and "buf_tail", which mark the live portion of the buffer.

o "buf_ackno", the Acknowledgement Number of the most recent packet acknowledged in the buffer. This corresponds to the "head" pointer.

o "buf_nonce", the one-bit sum (exclusive-or, or parity) of the ECN Nonces received on all packets acknowledged by the buffer with state 0 .

We draw acknowledgement buffers like this:

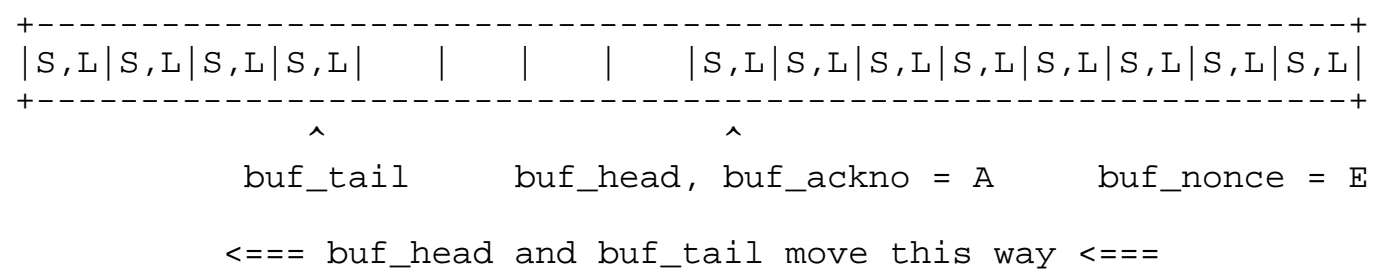


Each "S,L" represents a State/Run length byte. We will draw these buffers showing only their live portion and will add an annotation showing the Acknowledgement Number for the last live byte in the buffer. For example:

A $|S, L| S, L|S, L| S, L|S, L| S, L|S, L| S, L|S, L| S, L|S, L| S, L \mid T \quad$ BN $[E]$

Here, buf_nonce equals E and buf_ackno equals A.

We will use this buffer as a running example.

$10|0,0| 3,0|3,0| 3,0|0,4| 1,0|0,0| 0 \quad$ BN [1] [Example Buffer]

In concrete terms, its meaning is as follows:

Packet 10 was received. (The head of the buffer has sequence number 10 , state 0 , and run length 0. )

Packets 9, 8, and 7 have not yet been received. (The three bytes preceding the head each have state 3 and run length 0. )

Packets $6,5,4,3$, and 2 were received.

Packet 1 was ECN marked.

Packet 0 was received.

The one-bit sum of the ECN Nonces on packets $10,6,5,4,3,2$, and 0 equals 1 .

Additionally, the HC-Receiver must keep some information about the Ack Vectors it has recently sent. For each packet sent carrying an Ack Vector, it remembers four variables:

o "ack_seqno", the Sequence Number used for the packet. This is an HC-Receiver sequence number.

o "ack_ptr", the value of buf_head at the time of acknowledgement.

o "ack_runlen", the run length stored in the byte of buffer data at buf_head at the time of acknowledgement. 
o "ack_ackno", the Acknowledgement Number used for the packet. This is an HC-Sender sequence number. Since acknowledgements are cumulative, this single number completely specifies all necessary information about the packets acknowledged by this Ack Vector.

o "ack_nonce", the one-bit sum of the ECN Nonces for all state 0 packets in the buffer from buf_head to ack_ackno, inclusive. Initially, this equals the Nonce Echo of the acknowledgement's Ack Vector (or, if the ack packet contained more than one Ack Vector, the exclusive-or of all the acknowledgement's Ack Vectors). It changes as information about old acknowledgements is removed (so ack_ptr and buf_head diverge) and as old packets arrive (so they change from state 3 or state 1 to state 0 ).

\section{A.1. Packet Arrival}

This section describes how the HC-Receiver updates its acknowledgement buffer as packets arrive from the HC-Sender.

\section{A.1.1. New Packets}

When a packet with sequence Number greater than buf_ackno arrives, the HC-Receiver updates buf_head (by moving it to the left appropriately), buf_ackno (which is set to the new packet's Sequence Number), and possibly buf_nonce (if the packet arrived unmarked with ECN Nonce 1), in addition to the buffer itself. For example, if HC-Sender packet 11 arrived ECN marked, the Example Buffer above would enter this new state (changes are marked with stars):

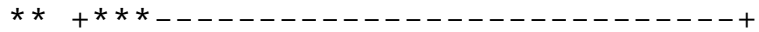

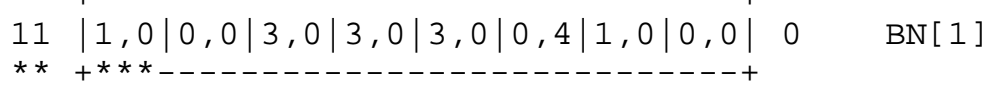

If the packet's state equals the state at the head of the buffer, the HC-Receiver may choose to increment its run length (up to the maximum). For example, if HC-Sender packet 11 arrived without ECN marking and with ECN Nonce 0, the Example Buffer might enter this state instead:

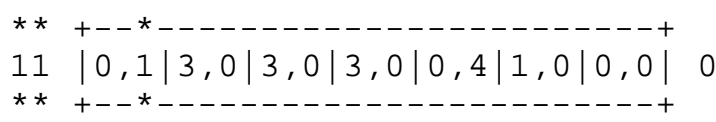

BN [1] 
Of course, the new packet's sequence number might not equal the expected sequence number. In this case, the HC-Receiver will enter the intervening packets as state 3. If several packets are missing, the HC-Receiver may prefer to enter multiple bytes with run length 0 , rather than a single byte with a larger run length; this simplifies table updates if one of the missing packets arrives. For example, if HC-Sender packet 12 arrived with ECN Nonce 1, the Example Buffer would enter this state:

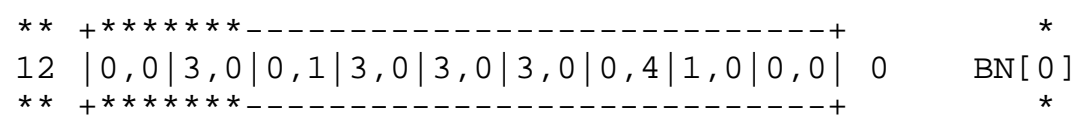

Of course, the circular buffer may overflow when the HC-Sender is sending data at a very high rate, when the HC-Receiver's acknowledgements are not reaching the HC-Sender, or when the HC-Sender is forgetting to acknowledge those acks (so the HC-Receiver is unable to clean up old state). In this case, the HC-Receiver should either compress the buffer (by increasing run lengths when possible), transfer its state to a larger buffer, or, as a last resort, drop all received packets, without processing them at all, until its buffer shrinks again.

\section{A.1.2. Old Packets}

When a packet with sequence Number $S<=$ buf_ackno arrives, the HC-Receiver will scan the table for the byte corresponding to $\mathrm{S}$. (Indexing structures could reduce the complexity of this scan.) If $S$ was previously lost (State 3), and it was stored in a byte with run length 0 , the HC-Receiver can simply change the byte's state. For example, if HC-Sender packet 8 was received with ECN Nonce 0 , the Example Buffer would enter this state:

$$
10|0,0| 3,0|0,0| 3,0|0,4| 1,0|0,0| 0 \quad \text { BN [1] }
$$

If $S$ was not marked as lost, or if it was not contained in the table, the packet is probably a duplicate and should be ignored. (The new packet's ECN marking state might differ from the state in the buffer; Section 11.4.1 describes what is allowed then.) If $S^{\prime} s$ buffer byte has a non-zero run length, then the buffer might need to be reshuffled to make space for one or two new bytes.

The ack_nonce fields may also need manipulation when old packets arrive. In particular, when s transitions from state 3 or state 1 to State 0 , and S had ECN Nonce 1, then the implementation should flip the value of ack_nonce for every acknowledgement with ack_ackno $>=S$. 
It is impossible with this data structure to shift packets from state 0 to state 1, since the buffer doesn't store individual packets' ECN Nonces.

\section{A.2. Sending Acknowledgements}

Whenever the HC-Receiver needs to generate an acknowledgement, the buffer's contents can simply be copied into one or more Ack Vector options. Copied Ack Vectors might not be maximally compressed; for example, the Example Buffer above contains three adjacent 3,0 bytes that could be combined into a single 3,2 byte. The HC-Receiver might, therefore, choose to compress the buffer in place before sending the option, or to compress the buffer while copying it; either operation is simple.

Every acknowledgement sent by the HC-Receiver SHOULD include the entire state of the buffer. That is, acknowledgements are cumulative.

If the acknowledgement fits in one Ack Vector, that Ack Vector's Nonce Echo simply equals buf_nonce. For multiple Ack Vectors, more care is required. The Ack Vectors should be split at points corresponding to previous acknowledgements, since the stored ack_nonce fields provide enough information to calculate correct Nonce Echoes. The implementation should therefore acknowledge data at least once per 253 bytes of buffer state. (Otherwise, there'd be no way to calculate a Nonce Echo.)

For each acknowledgement it sends, the HC-Receiver will add an acknowledgement record. ack_seqno will equal the HC-Receiver sequence number it used for the ack packet; ack_ptr will equal buf_head; ack_runlen will equal the run length stored in the buffer's buf_head byte; ack_ackno will equal buf_ackno; and ack_nonce will equal buf_nonce.

\section{A.3. Clearing state}

Some of the HC-Sender's packets will include acknowledgement numbers, which ack the HC-Receiver's acknowledgements. When such an ack is received, the HC-Receiver finds the acknowledgement record $\mathrm{R}$ with the appropriate ack_seqno and then does the following:

- If the run length in the buffer's R.ack_ptr byte is greater than R.ack_runlen, then it decrements that run length by R.ack_runlen +1 and sets buf_tail to R.ack_ptr. Otherwise, it sets buf_tail to R.ack_ptr + 1 . 
o If R.ack_nonce is 1, it flips buf_nonce, and the value of ack_nonce for every later ack record.

o It throws away $\mathrm{R}$ and every preceding ack record.

(The HC-Receiver may choose to keep some older information, in case a lost packet shows up late.) For example, say that the HC-Receiver storing the Example Buffer had sent two acknowledgements already:

1. ack_seqno $=59$, ack_runlen $=1$, ack_ackno $=3$, ack_nonce $=1$.

2. ack_seqno $=60$, ack_runlen $=0$, ack_ackno $=10$, ack_nonce $=0$.

Say the HC-Receiver then received a DCCP-DataAck packet with Acknowledgement Number 59 from the HC-Sender. This informs the HC-Receiver that the HC-Sender received, and processed, all the information in HC-Receiver packet 59. This packet acknowledged HC-Sender packet 3, so the HC-Sender has now received HC-Receiver's acknowledgements for packets $0,1,2$, and 3. The Example Buffer should enter this state:

$$
10 \underset{\mid}{|0,0| 3,0|3,0| 3,0|0,2|} 4 \quad \text { * } 4 \text { [ }
$$

The tail byte's run length was adjusted, since packet 3 was in the middle of that byte. Since R.ack_nonce was 1, the buf_nonce field was flipped, as were the ack_nonce fields for later acknowledgements (here, the HC-Receiver Ack 60 record, not shown, has its ack_nonce flipped to 1). The HC-Receiver can also throw away stored information about HC-Receiver Ack 59 and any earlier acknowledgements.

A careful implementation might try to ensure reasonable robustness to reordering. Suppose that the Example Buffer is as before, but that packet 9 now arrives, out of sequence. The buffer would enter this state:

$$
10|0,0| 0,0|3,0| 3,0|0,4| 1,0|0,0| 0 \quad \text { BN [1] }
$$

The danger is that the HC-Sender might acknowledge the HC-Receiver's previous acknowledgement (with sequence number 60), which says that Packet 9 was not received, before the HC-Receiver has a chance to send a new acknowledgement saying that Packet 9 actually was received. Therefore, when packet 9 arrived, the HC-Receiver might modify its acknowledgement record as follows: 
1. ack_seqno $=59$, ack_ackno $=3$, ack_nonce $=1$.

2. ack_seqno $=60$, ack_ackno $=3$, ack_nonce $=1$.

That is, Ack 60 is now treated like a duplicate of Ack 59. This would prevent the Tail pointer from moving past packet 9 until the HC-Receiver knows that the HC-Sender has seen an Ack Vector indicating that packet's arrival.

\section{A.4. Processing Acknowledgements}

When the HC-Sender receives an acknowledgement, it generally cares about the number of packets that were dropped and/or ECN marked. It simply reads this off the Ack Vector. Additionally, it should check the ECN Nonce for correctness. (As described in Section 11.4.1, it may want to keep more detailed information about acknowledged packets in case packets change states between acknowledgements, or in case the application queries whether a packet arrived.)

The HC-Sender must also acknowledge the HC-Receiver's acknowledgements so that the HC-Receiver can free old Ack Vector state. (Since Ack Vector acknowledgements are reliable, the HC-Receiver must maintain and resend Ack Vector information until it is sure that the HC-Sender has received that information.) A simple algorithm suffices: since Ack Vector acknowledgements are cumulative, a single acknowledgement number tells HC-Receiver how much ack information has arrived. Assuming that the HC-Receiver sends no data, the HC-Sender can ensure that at least once a round-trip time, it sends a DCCP-DataAck packet acknowledging the latest DCCP-Ack packet it has received. Of course, the HC-Sender only needs to acknowledge the HC-Receiver's acknowledgements if the HC-Sender is also sending data. If the HC-Sender is not sending data, then the HC-Receiver's Ack Vector state is stable, and there is no need to shrink it. The HC-Sender must watch for drops and ECN marks on received DCCP-Ack packets so that it can adjust the HC-Receiver's ack-sending rate in response to congestion, for example, with Ack Ratio.

If the other half-connection is not quiescent -- that is, the HC-Receiver is sending data to the HC-Sender, possibly using another CCID -- then the acknowledgements on that half-connection are sufficient for the HC-Receiver to free its state. 


\section{B. Appendix: Partial Checksumming Design Motivation}

A great deal of discussion has taken place regarding the utility of allowing a DCCP sender to restrict the checksum so that it does not cover the complete packet. This section attempts to capture some of the rationale behind specific details of DCCP design.

Many of the applications that we envisage using DCCP are resilient to some degree of data loss, or they would typically have chosen a reliable transport. Some of these applications may also be resilient to data corruption -- some audio payloads, for example. These resilient applications might rather receive corrupted data than have DCCP drop corrupted packets. This is particularly because of congestion control: DCCP cannot tell the difference between packets dropped due to corruption and packets dropped due to congestion, and so it must reduce the transmission rate accordingly. This response may cause the connection to receive less bandwidth than it is due; corruption in some networking technologies is independent of, or at least not always correlated to, congestion. Therefore, corrupted packets do not need to cause as strong a reduction in transmission rate as the congestion response would dictate (as long as the DCCP header and options are not corrupt).

Thus DCCP allows the checksum to cover all of the packet, just the DCCP header, or both the DCCP header and some number of bytes from the application data. If the application cannot tolerate any data corruption, then the checksum must cover the whole packet. If the application would prefer to tolerate some corruption rather than have the packet dropped, then it can set the checksum to cover only part of the packet (but always the DCCP header). In addition, if the application wishes to decouple checksumming of the DCCP header from checksumming of the application data, it may do so by including the Data Checksum option. This would allow DCCP to discard corrupted application data without mistaking the corruption for network congestion.

Thus, from the application point of view, partial checksums seem to be a desirable feature. However, the usefulness of partial checksums depends on partially corrupted packets being delivered to the receiver. If the link-layer CRC always discards corrupted packets, then this will not happen, and so the usefulness of partial checksums would be restricted to corruption that occurred in routers and other places not covered by link CRCs. There does not appear to be consensus on how likely it is that future network links that suffer significant corruption will not cover the entire packet with a single strong CRC. DCCP makes it possible to tailor such links to the application, but it is difficult to predict if this will be compelling for future link technologies. 
In addition, partial checksums do not co-exist well with IP-level authentication mechanisms such as IPsec $A H$, which cover the entire packet with a cryptographic hash. Thus, if cryptographic authentication mechanisms are required to co-exist with partial checksums, the authentication must be carried in the application data. A possible mode of usage would appear to be similar to that of Secure RTP. However, such "application-level" authentication does not protect the DCCP option negotiation and state machine from forged packets. An alternative would be to use IPsec ESP, and to use encryption to protect the DCCP headers against attack, while using the DCCP header validity checks to authenticate that the header is from someone who possessed the correct key. While this is resistant to replay (due to the DCCP sequence number), it is not by itself resistant to some forms of man-in-the-middle attacks because the application data is not tightly coupled to the packet header. Thus, an application-level authentication probably needs to be coupled with IPsec ESP or a similar mechanism to provide a reasonably complete security solution. The overhead of such a solution might be unacceptable for some applications that would otherwise wish to use partial checksums.

On balance, the authors believe that DCCP partial checksums have the potential to enable some future uses that would otherwise be difficult. As the cost and complexity of supporting them is small, it seems worth including them at this time. It remains to be seen whether they are useful in practice.

Normative References

[RFC793] Postel, J., "Transmission Control Protocol", STD 7, RFC 793, September 1981.

[RFC1191] Mogul, J. and S. Deering, "Path MTU discovery", RFC 1191, November 1990.

[RFC2119] Bradner, S., "Key words for use in RFCs to Indicate Requirement Levels", BCP 14, RFC 2119, March 1997.

[RFC2434] Narten, T. and H. Alvestrand, "Guidelines for Writing an IANA Considerations section in RFCs", BCP 26, RFC 2434, October 1998 .

[RFC2460] Deering, S. and R. Hinden, "Internet Protocol, Version 6 (IPv6) Specification", RFC 2460, December 1998.

[RFC3168] Ramakrishnan, K., Floyd, S., and D. Black, "The Addition of Explicit Congestion Notification (ECN) to IP", RFC 3168, September 2001. 
[RFC3309] Stone, J., Stewart, R., and D. Otis, "Stream Control Transmission Protocol (SCTP) Checksum Change", RFC 3309, September 2002 .

[RFC3692] Narten, T., "Assigning Experimental and Testing Numbers Considered Useful", BCP 82, RFC 3692, January 2004 .

[RFC3775] Johnson, D., Perkins, C., and J. Arkko, "Mobility Support in IPv6", RFC 3775, June 2004.

[RFC3828] Larzon, L-A., Degermark, M., Pink, S., Jonsson, L-E., and G. Fairhurst, "The Lightweight User Datagram Protocol (UDP-Lite)", RFC 3828, July 2004.

Informative References

[B98] Bellovin, S.M., "Cryptography and the Internet", CRYPTO'98 (LNCS 1462), pp 46-55, August 1988 .

[BB01] Bellovin, S.M. and M. Blaze, "Cryptographic Modes of Operation for the Internet", 2nd NIST Workshop on Modes of Operation, August 2001.

[M85] Morris, R.T., "A Weakness in the 4.2BSD Unix TCP/IP Software", Computer Science Technical Report 117, AT\&T Bell Laboratories, Murray Hill, NJ, February 1985.

[PMTUD] Mathis, M. and J. Heffner, "Path MTU Discovery", Work in Progress, March 2006.

[RFC792] Postel, J., "Internet Control Message Protocol", STD 5, RFC 792, September 1981.

[RFC1812] Baker, F., "Requirements for IP Version 4 Routers", RFC 1812, June 1995.

[RFC1948] Bellovin, S., "Defending Against Sequence Number Attacks", RFC 1948, May 1996.

[RFC1982] Elz, R. and R. Bush, "Serial Number Arithmetic", RFC 1982, August 1996.

[RFC2018] Mathis, M., Mahdavi, J., Floyd, S., and A. Romanow, "TCP Selective Acknowledgement Options", RFC 2018, October 1996 . 
[RFC2401]

[RFC2463]

[RFC2581]

$[\mathrm{RFC} 2960]$

$[\mathrm{RFC} 3124]$

[RFC3360]

[RFC3448]

$[\mathrm{RFC} 3540]$

$[\mathrm{RFC} 3550]$

[RFC3611]

$[\operatorname{RFC} 3711]$

[RFC3819]
Kent, S. and R. Atkinson, "Security Architecture for the Internet Protocol", RFC 2401, November 1998.

Conta, A. and S. Deering, "Internet Control Message Protocol (ICMPV6) for the Internet Protocol Version 6 (IPv6) Specification", RFC 2463, December 1998.

Allman, M., Paxson, V., and W. Stevens, "TCP Congestion Control", RFC 2581, April 1999.

Stewart, R., Xie, Q., Morneault, K., Sharp, C., Schwarzbauer, H., Taylor, T., Rytina, I., Kalla, M., Zhang, L., and V. Paxson, "Stream Control Transmission Protocol", RFC 2960, October 2000.

Balakrishnan, H. and S. Seshan, "The Congestion Manager", RFC 3124, June 2001.

Floyd, S., "Inappropriate TCP Resets Considered Harmful", BCP 60, RFC 3360, August 2002.

Handley, M., Floyd, S., Padhye, J., and J. Widmer, "TCP Friendly Rate Control (TFRC): Protocol Specification", RFC 3448, January 2003.

Spring, N., Wetherall, D., and D. Ely, "Robust Explicit Congestion Notification (ECN) Signaling with Nonces", RFC 3540, June 2003.

Schulzrinne, H., Casner, S., Frederick, R., and V. Jacobson, "RTP: A Transport Protocol for Real-Time Applications", STD 64, RFC 3550, July 2003.

Friedman, T., Caceres, R., and A. Clark, "RTP Control Protocol Extended Reports (RTCP XR)", RFC 3611, November 2003.

Baugher, M., McGrew, D., Naslund, M., Carrara, E., and K. Norrman, "The Secure Real-time Transport Protocol (SRTP) ", RFC 3711, March 2004.

Karn, P., Bormann, C., Fairhurst, G., Grossman, D., Ludwig, R., Mahdavi, J., Montenegro, G., Touch, J., and L. Wood, "Advice for Internet Subnetwork Designers", BCP 89, RFC 3819, July 2004. 
[RFC4086] Eastlake, D., 3rd, Schiller, J., and S. Crocker, "Randomness Requirements for security", BCP 106, RFC 4086, June 2005 .

[RFC4341] Floyd, S. and E. Kohler, "Profile for Datagram Congestion Control Protocol (DCCP) Congestion Control ID 2: TCP-like Congestion Control", RFC 4341, March 2006 .

[RFC4342] Floyd, S., Kohler, E., and J. Padhye, "Profile for Datagram Congestion Control Protocol (DCCP) Congestion Control ID 3: TCP-Friendly Rate Control (TFRC)", RFC 4342 , March 2006.

[SHHP00] Spatscheck, O., Hansen, J.S., Hartman, J.H., and L.L. Peterson, "Optimizing TCP Forwarder Performance", IEEE/ACM Transactions on Networking 8(2):146-157, April 2000 .

[SYNCOOKIES] Bernstein, D.J., "SYN Cookies", http://cr.yp.to/syncookies.html, as of March 2006.

[VBK0 5 ]

Vanit-Anunchai, S., Billington, J., and T. Kongprakaiwoot, "Discovering Chatter and Incompleteness in the Datagram Congestion Control Protocol", FORTE 2005, pp 143-158, October 2005. 


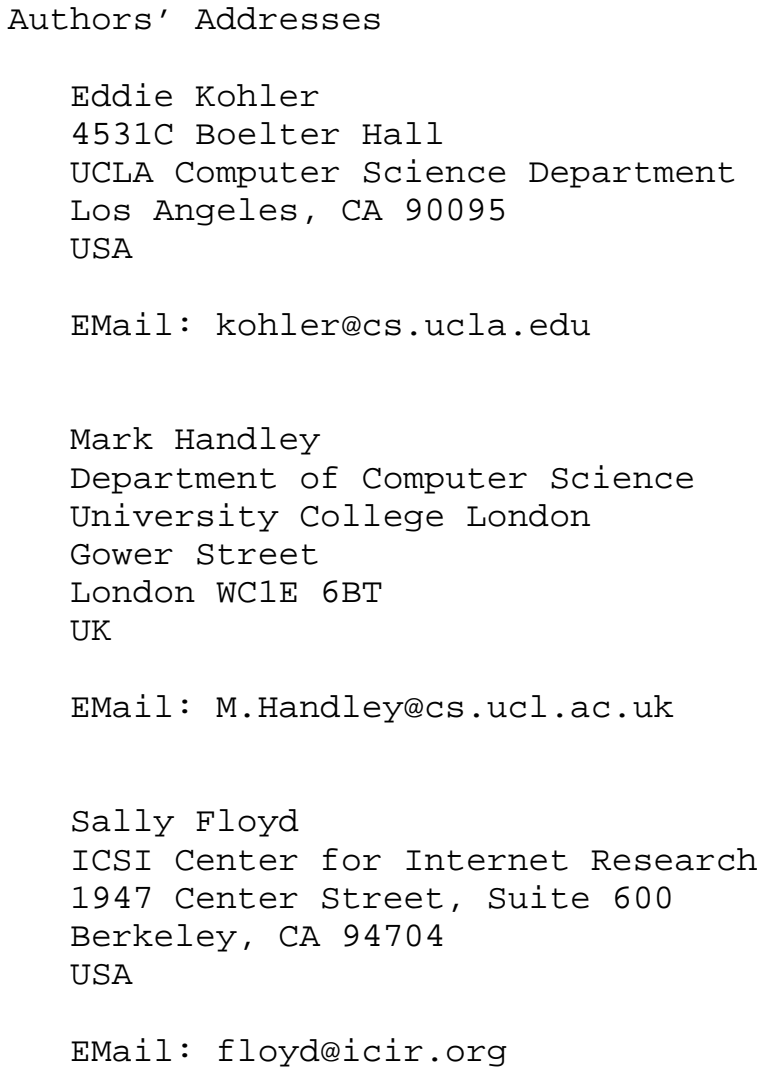

EMail: floydeicir.org 
Full Copyright statement

Copyright (C) The Internet Society (2006).

This document is subject to the rights, licenses and restrictions contained in BCP 78, and except as set forth therein, the authors retain all their rights.

This document and the information contained herein are provided on an "AS IS" basis and THE CONTRIBUTOR, THE ORGANIZATION HE/SHE REPRESENTS OR IS SPONSORED BY (IF ANY), THE INTERNET SOCIETY AND THE INTERNET ENGINEERING TASK FORCE DISCLAIM ALL WARRANTIES, EXPRESS OR IMPLIED, INCLUDING BUT NOT LIMITED TO ANY WARRANTY THAT THE USE OF THE INFORMATION HEREIN WILL NOT INFRINGE ANY RIGHTS OR ANY IMPLIED WARRANTIES OF MERCHANTABILITY OR FITNESS FOR A PARTICULAR PURPOSE.

Intellectual Property

The IETF takes no position regarding the validity or scope of any Intellectual property Rights or other rights that might be claimed to pertain to the implementation or use of the technology described in this document or the extent to which any license under such rights might or might not be available; nor does it represent that it has made any independent effort to identify any such rights. Information on the procedures with respect to rights in RFC documents can be found in BCP 78 and BCP 79 .

Copies of IPR disclosures made to the IETF Secretariat and any assurances of licenses to be made available, or the result of an attempt made to obtain a general license or permission for the use of such proprietary rights by implementers or users of this specification can be obtained from the IETF on-line IPR repository at http://www.ietf.org/ipr.

The IETF invites any interested party to bring to its attention any copyrights, patents or patent applications, or other proprietary rights that may cover technology that may be required to implement this standard. Please address the information to the IETF at ietfipreietf.org.

Acknowledgement

Funding for the RFC Editor function is provided by the IETF Administrative Support Activity (IASA) . 